\title{
Weibull Survival Model and Its Posterior Analysis
}

\author{
G. Venkatesan ${ }^{1}$, P. Saranya ${ }^{2}$ \\ ${ }^{1}$ Department of Statistics, Government Arts College (Autonomous), Salem-7, Tamil Nadu, India \\ ${ }^{2}$ Department of Statistics, Government Arts College (Autonomous), Salem-7, Tamil Nadu, India \\ "Corresponding author: nsabsa08@gmail.com
}

Available online at: www.isroset.org

Received: 08/Oct/2018, Accepted:17/Oct/2018, Online: 31/Oct/2018

\begin{abstract}
In this paper, the two parameter Weibull distribution is considered for estimating the Bayes risk of survival function assuming non-informative and informative priors such as Jeffrey's, Extension of Jeffrey's, Lognormal-Inverted Gamma and Gamma-Gamma using various type of loss functions with Squared error loss, Linex loss, General entropy loss, Quadratic loss, Weighted loss, Precautionary loss and Squared logarithmic loss. Posterior analysis done through estimating Bayes risk of survival function for combination of priors with loss functions. To illustrate the methodology, simulation study is carried out and done the parametric analysis.
\end{abstract}

Keywords: Bayes risk, Weibull distribution, Squared error loss function, General entropy loss function, Linex loss function, Precautionary loss function, Quadratic loss function, Squared logarithmic loss function, Weighted loss function.

\section{INTRODUCTION}

In real life situation, Weibull distribution is commonly used as a lifetime distribution in reliability and survival applications. The primary advantage of Weibull analysis is the ability to provide precise failure measures and forecasts with small samples. A sample with small size is also allowed in cost effective component testing. Sinha, S.K, (1986) obtained the Bayes estimate of reliability function and the hazard rate of the Weibull distribution by assigning squared error loss function. Al Omari Mohammed Ahmed and et al., (2010) compared the Bayesian and maximum likelihood estimation for Weibull distribution using Jeffrey's and Extension of Jeffrey's priors. Al Omari Mohammed Ahmed and et al., (2011) studied the Bayesian survival estimator with censored data using Jeffrey's prior and Extension of Jeffrey's prior information. Pandey, B.N and et al., (2011) compared the Bayesian and ML estimate of scale parameter of the Weibull distribution under Linex loss function. Chris Bambay Guure and et al., (2014) have studied the performance of Bayes and frequentist estimators for the two parameter Weibull failure time distribution by using non-informative prior and also the reliability and hazard functions were derived under Squared error loss, General entropy loss and Linex loss functions. Lavanya, A. and T. Leo Alexander (2016) have studied the Bayesian estimation of survival function under the Constant Shape Bi-Weibull distribution by using Extension of Jeffrey's prior under the losses such as Squared error loss, General entropy loss and Linex loss functions. Venkatesan, G and P.Saranya (2018a) have studied the performance of maximum likelihood estimation and Bayesian estimation of survival function of two parameter Weibull distribution assuming informative prior under Squared error loss, General entropy loss and Linex loss functions. Venkatesan, G and P.Saranya (2018b) carried out the Bayes risk of survival analysis through Weibull distribution using non-informative and informative priors as Jeffrey's, Extension of Jeffrey's, Lognormal-Inverted Gamma priors under Squared error loss, General entropy loss, Quadratic loss, Weighted loss and Squared logarithmic loss functions. Venkatesan,G and P.Saranya (2018c) studied the posterior risk of survival analysis for Weibull distribution using noninformative and informative priors as Extension of Jeffrey's, Lognormal-Inverted Gamma and Exponential-Gamma priors under Quadratic loss, Weighted loss, Squared logarithmic loss and Precautionary loss functions.

In this paper, we proposed to obtain Bayes risk of survival function for two parameter Weibull distribution using noninformative and informative priors such as Jeffrey's, Extension of Jeffrey's, Lognormal-Inverted Gamma and Gamma-Gamma priors under Squared error loss function (SELF), Linex loss function (LLF), General entropy loss function (GELF), Quadratic loss function (QLF), Weighted loss function (WLF), Precautionary loss function (PLF) and Squared logarithmic loss function (SLLF). Bayes risk analysis is done to find which combination of prior and loss function contribute the minimum risk among the all considered cases in this analysis. 
The layout of the paper follows as in section II, Maximum likelihood estimate of the parameters are obtained. In section III, Bayesian estimator is obtained with non-informative and informative priors. Further, in section IV, obtained the Bayes risk of survival function using with different loss functions. The simulation study is carried out in section $\mathrm{V}$ and conclusion with future study is reported in section VI.

\section{MAXIMUM LIKELIHOOD ESTIMATION}

Let $t_{1}, t_{2}, \ldots, t_{n}$ be a random sample of size $\mathrm{n}$ from Weibull distribution with scale parameter $(\beta)$ and shape parameter $(\alpha)$. The probability density function is

$$
f(t ; \alpha, \beta)=\frac{\alpha}{\beta} t^{\alpha-1} \exp \left(\frac{-t^{\alpha}}{\beta}\right), t>0, \alpha>0, \beta>0
$$

The cumulative distribution function is

$$
F(t ; \alpha, \beta)=1-\exp \left(\frac{-t^{\alpha}}{\beta}\right), t>0, \alpha>0, \beta>0
$$

The survival function of $t$ is

$$
S(t ; \alpha, \beta)=\exp \left(\frac{-t^{\alpha}}{\beta}\right)
$$

The likelihood function of $\mathrm{t}$ is

$$
L(t ; \alpha, \beta)=\prod_{i=1}^{n}\left\{\frac{\alpha}{\beta} t_{i}^{\alpha-1} \exp \left(\frac{-t_{i}^{\alpha}}{\beta}\right)\right\}
$$

Using the principle of MLE, we get

$$
\hat{\beta}=\frac{1}{n} \sum_{i=1}^{n} t_{i}^{\widehat{\alpha}}
$$

where $\hat{\alpha}$ can be determined by using Newton-Raphson method and taking the initial value of $\alpha$ as $\alpha_{i}$ as per our convenience and iterating the process till it converges.

$$
\alpha_{i+1}=\alpha_{i}-\frac{\frac{n}{\alpha}+\sum_{i=1}^{n} \log t_{i}-\frac{\sum_{i=1}^{n} t_{i}{ }^{\alpha} \log t_{i}}{\frac{1}{n} \sum_{i=1}^{n} t_{i}{ }^{\alpha}}}{-\left\{\frac{n}{\alpha^{2}}+\frac{\sum_{i=1}^{n} t_{i}^{\alpha}\left(\log _{i}\right)^{2}}{\frac{1}{n} \sum_{i=1}^{n} t_{i}{ }^{\alpha}}\right\}}
$$

The estimate of survival function of Weibull distribution under the MLE is

$$
\hat{S}\left(t_{i}\right)=\exp \left[-\left(\frac{t_{i}^{\widehat{\alpha}}}{\widehat{\beta}}\right)\right]
$$

\section{BAYESIAN ESTIMATION}

The posterior distribution of the parameter can be obtained by multiplying likelihood function and prior of the parameters. Bayesian estimation of the parameter can be obtained by using posterior distribution instead of likelihood function. Bayesian estimation approach has received a lot of attention for analyzing failure time data. When prior knowledge about the parameter is not available, it is possible to make use of the non-informative prior. When we have knowledge on the parameter, the informative prior is preferred to use for the analysis.

\section{A. Jeffrey's prior}

The Jeffrey's prior for the Weibull distribution is

$$
v_{1}(\alpha, \beta) \propto\left(\frac{1}{\alpha \beta}\right)
$$

The posterior distribution of the parameters $\alpha$ and $\beta$ is obtained by multiplying the equations (4) and (8) as

$$
\pi_{1}(\alpha, \beta) \propto\left(\frac{1}{\alpha \beta}\right) \prod_{i=1}^{n}\left\{\frac{\alpha}{\beta} t^{\alpha-1} \exp \left(\frac{-t^{\alpha}}{\beta}\right)\right\}
$$

B.Extension of Jeffrey's prior

The Extension of Jeffrey's prior for the Weibull distribution is

$$
v_{2}(\alpha, \beta) \propto\left(\frac{1}{\alpha \beta}\right)^{2 p}, p \in R^{+}
$$

The posterior distribution of the parameters $\alpha$ and $\beta$ is obtained by multiplying the equations (4) and (10) as

$$
\pi_{2}(\alpha, \beta) \propto\left(\frac{1}{\alpha \beta}\right)^{2 p} \prod_{i=1}^{n}\left\{\frac{\alpha}{\beta} t^{\alpha-1} \exp \left(\frac{-t^{\alpha}}{\beta}\right)\right\}
$$

where $\alpha, \beta$ and $p$ are described in equations (4) and (10).Equations 


\section{C.Lognormal-Inverted Gamma prior}

The shape parameter $\alpha$ follows Lognormal distribution with hyperparameter $\mathrm{c}$ and scale parameter $\beta$ follows Inverted Gamma distribution with hyperparameters a and $b$. The joint prior distribution of $\alpha$ and $\beta$ is

$$
v_{3}(\alpha, \beta) \propto \frac{1}{\alpha} \exp \left\{\frac{-(\log \alpha)^{2}}{2 c^{2}}\right\}\left(\frac{1}{\beta}\right)^{a+1} \exp \left(\frac{-b}{\beta}\right), \quad \alpha, \beta>0 ; a, b, c>0
$$

The posterior distribution of the parameters $\alpha$ and $\beta$ is obtained by multiplying the equations (4) and (12) as

$$
\pi_{3}(\alpha, \beta) \propto\left(\frac{1}{\alpha}\right) \exp \left\{\frac{-(\log \alpha)^{2}}{2 c^{2}}\right\}\left(\frac{1}{\beta}\right)^{a+1} \exp \left(\frac{-b}{\beta}\right) \prod_{i=1}^{n}\left\{\frac{\alpha}{\beta} t^{\alpha-1} \exp \left(\frac{-t^{\alpha}}{\beta}\right)\right\}
$$

where $\alpha, \beta, a, b$ and $c$ are described in equations (4) and (12).

\section{D.Gamma-Gamma prior}

The shape parameter $\alpha$ follows Gamma distribution with hyperparameters $a_{1}$ and $b_{1}$ and scale parameter $\beta$ follows Gamma distribution with hyperparameter $c_{1}$ and $d_{1}$. The joint prior distribution of $\alpha$ and $\beta$ is

$$
v_{4}(\alpha, \beta) \propto \alpha^{a_{1}-1} \exp \left(-b_{1} \alpha\right) \beta^{d_{1}-1} \exp \left(-c_{1} \beta\right), \quad \alpha, \beta>0 ; a_{1}, b_{1}, c_{1}, d_{1}>0
$$

The posterior distribution of the parameters $\alpha$ and $\beta$ is obtained by multiplying the equation (4) and (14) is

$$
\pi_{4}(\alpha, \beta) \propto \alpha^{a_{1}-1} \exp \left(-b_{1} \alpha\right) \beta^{d_{1}-1} \exp \left(-c_{1} \beta\right) \prod_{i=1}^{n}\left\{\frac{\alpha}{\beta} t^{\alpha-1} \exp \left(\frac{-t^{\alpha}}{\beta}\right)\right\}
$$

where

$\begin{array}{cccc}\text { Loss Function } & \text { Expression } & \text { Bayes estimator } & \text { Bayes Risk } \\ \text { SELF } & L(\hat{\theta}, \theta)=(\hat{\theta}-\theta)^{2} & E(\theta) & E\left(\theta^{2}\right)-[E(\theta)]^{2} \\ \text { LLF } & L(\hat{\theta}, \theta)=e^{m(\hat{\theta}-\theta)}-m(\hat{\theta}-\theta)-1 & \frac{-1}{m} \log \left[E\left(e^{-m \theta}\right)\right] & \log \left[E\left(e^{-m \theta}\right)\right]+m E(\theta) \\ \text { GELF } & L(\theta, \hat{\theta})=\left(\frac{\hat{\theta}}{\theta}\right)^{k}-k \log \left(\frac{\hat{\theta}}{\theta}\right)-1 & {\left[E\left(\theta^{-k}\right)\right]^{-1} k} & k E(\log \theta)+\log \left[E\left(\theta^{-k}\right)\right] \\ \text { QLF } & L(\theta, \hat{\theta})=\left(1-\frac{\hat{\theta}}{\theta}\right)^{2} & \frac{E\left(\theta^{-1}\right)}{E\left(\theta^{-2}\right)} & 1-\frac{\left[E\left(\theta^{-1)}\right]^{2}\right.}{E\left(\theta^{-2}\right)} \\ \text { WLF } & L(\hat{\theta}, \theta)=\frac{(\hat{\theta}-\theta)^{2}}{\theta} & {\left[E\left(\theta^{-1}\right)\right]^{-1}} & E(\theta)-\left[E\left(\theta^{-1)}\right]^{-1}\right. \\ \text { PLF } & L(\hat{\theta}, \theta)=\frac{(\hat{\theta}-\theta)^{2}}{\hat{\theta}} & \sqrt{E\left(\theta^{2}\right)} & \left.2\left\{\sqrt{E\left(\theta^{2}\right.}\right)-E(\theta)\right\} \\ \text { SLLF } & L(\theta, \hat{\theta})=(\log \hat{\theta}-\log \theta)^{2} & \exp [E(\log \theta)] & E\left[(\log \theta)^{2}\right]-[E(\log \theta)]^{2}\end{array}$

$\alpha, \beta, a_{1}, b_{1}, c_{1}$ and $d_{1}$ are described in equations (4) and (14).

\section{BAYES RiSk OF SURVIVAl FUnCTION UNDER DIFFERENT LoSS FUNCTION}

We consider the Squared Error loss, Linex loss, General entropy loss, Quadratic loss, Weighted loss, Precautionary loss and Squared Logarithmic loss functions. The following table shows the expression of Bayesian estimator and Bayes risk with different loss functions for the parameter.

\section{A.Jeffrey's prior under Linex loss function}

The Bayes risk of survival function using Jeffrey's prior under Linex loss function is

$$
R\left[\hat{S}\left(t_{i}\right)\right]_{B L}=\log \left\{E\left(e^{-m\left\{\exp \left[-\left(\frac{t_{i}{ }^{\alpha}}{\beta}\right)\right]\right\}}\right)\right\}+m E\left\{\exp \left[-\left(\frac{t_{i}{ }^{\alpha}}{\beta}\right)\right]\right\}=\log \left\{\frac{\iint e^{-m\left\{\exp \left[-\left(\frac{t_{i}{ }^{\alpha}}{\beta}\right)\right]\right\}_{\pi_{1}(\alpha, \beta) d \beta d \alpha}}}{\iint \pi_{1}(\alpha, \beta) d \beta d \alpha}\right\}+m\left\{\frac{\iint \exp \left[-\left(\frac{t_{i}{ }^{\alpha}}{\beta}\right)\right] \pi_{1}(\alpha, \beta) d \beta d \alpha}{\iint \pi_{1}(\alpha, \beta) d \beta d \alpha}\right\}
$$

The above equation contains a ratio of two integrals which cannot be solved analytically, so we use Lindley's approximation procedure to estimate the survival function.

Using Lindley's approximation, the expansion of $\frac{\int u(\theta) v(\theta) \exp [L(\theta)] d \theta}{\int v(\theta) \exp [L(\theta)] d \theta}$ can be performed as

$$
\hat{\theta}=u+\frac{1}{2}\left[u_{11} \sigma_{11}+u_{22} \sigma_{22}\right]+u_{1} \rho_{1} \sigma_{11}+u_{2} \rho_{2} \sigma_{22}+\frac{1}{2}\left[L_{30} u_{1} \sigma^{2}{ }_{11}+L_{03} u_{2} \sigma_{22}^{2}\right]
$$

where $\mathrm{L}$ is the log likelihood function and $\rho$ is the logarithmic of prior distribution.

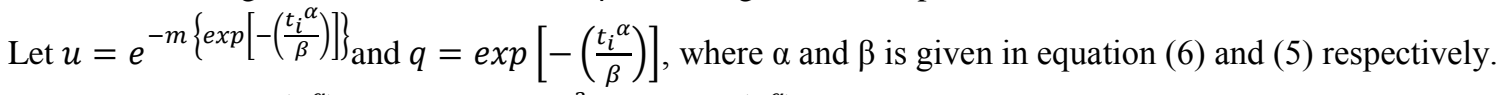

$u_{1}=\frac{d u}{d \beta}=-m q u\left(\frac{t_{i}{ }^{\alpha}}{\beta^{2}}\right) ; \quad u_{11}=\frac{d^{2} u}{d \beta^{2}}=m q u\left(\frac{t_{i}{ }^{\alpha}}{\beta^{4}}\right)\left(m q t_{i}{ }^{\alpha}-t_{i}{ }^{\alpha}+2 \beta\right) ;$

$u_{2}=\frac{d u}{d \alpha}=m q u\left(\frac{t_{i} \log _{i}}{\beta}\right) ; \quad u_{22}=\frac{d^{2} u}{d \alpha^{2}}=m q u\left(\frac{t_{i}\left(\log t_{i}\right)^{2}}{\beta}\right)\left(1+m q \frac{t_{i}^{\alpha}}{\beta}-\frac{t_{i}^{\alpha}}{\beta}\right)$ 
$\rho(\alpha, \beta)=-\log \alpha-\log \beta ; \rho_{1}=\frac{d u}{d \beta}=-\frac{1}{\beta} ; \quad \rho_{2}=\frac{d u}{d \alpha}=-\frac{1}{\alpha}$

$\sigma_{11}=\left(-L_{20}\right)^{-1} ; \sigma_{22}=\left(-L_{02}\right)^{-1} ; L_{02}=\frac{d^{2} L}{d \alpha^{2}}=-\frac{n}{\alpha^{2}}-\frac{1}{\beta} \sum_{i=1}^{n} t_{i}{ }^{\alpha}\left(\log t_{i}\right)^{2} ; L_{03}=\frac{d^{3} L}{d \alpha^{3}}=\frac{2 n}{\alpha^{3}}-\frac{1}{\beta} \sum_{i=1}^{n} t_{i}{ }^{\alpha}\left(\log t_{i}\right)^{3} L_{20}=$ $\frac{d^{2} L}{d \beta^{2}}=\frac{n}{\beta^{2}}-\frac{2}{\beta^{3}} \sum_{i=1}^{n} t_{i}^{\alpha} ; L_{30}=\frac{d^{3} L}{d \beta^{3}}=-\frac{2 n}{\beta^{3}}+\frac{6}{\beta^{4}} \sum_{i=1}^{n} t_{i}^{\alpha}$

Let $u=\exp \left[-\left(\frac{t_{i}^{\alpha}}{\beta}\right)\right]$ and $e=-\left(\frac{t_{i}^{\alpha}}{\beta}\right) ; u_{1}=\frac{d u}{d \beta}=\frac{-u e}{\beta} ; u_{11}=\frac{d^{2} u}{d \beta^{2}}=\frac{u e}{\beta^{2}}(e+2)$

$u_{2}=\frac{d u}{d \alpha}=u e\left(\log t_{i}\right) ; u_{22}=\frac{d^{2} u}{d \alpha^{2}}=2 u e\left(\log t_{i}\right)^{2}(1+e)$

The Bayes risk of survival function using Jeffrey's prior under Linex loss function $R\left[\hat{S}\left(t_{i}\right)\right]_{B L}$ is

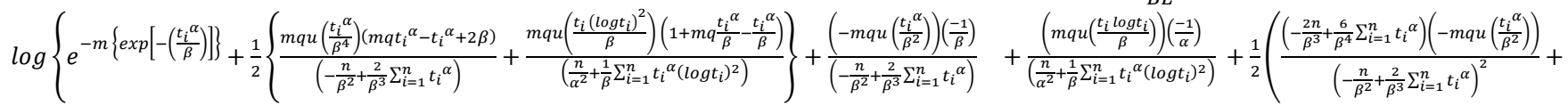

$\left.\left.\frac{\left[\frac{2 n}{\alpha^{3}}-\frac{1}{\beta} \sum_{i=1}^{n} t_{i}^{\alpha}\left(\log _{i}\right)^{3}\right]\left[\operatorname{mqu}\left(\frac{t_{i} \log t_{i}}{\beta}\right)\right]}{\left[\frac{n}{\alpha^{2}}+\frac{1}{\beta} \sum_{i=1}^{n} t_{i}{ }^{\alpha}\left(\log _{i}\right)^{2}\right]^{2}}\right)\right\}+$

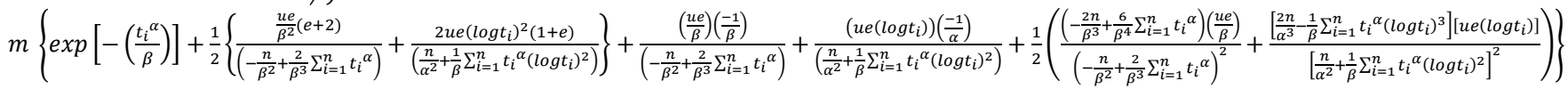

B.Jeffrey's prior under Precautionary loss function

The Bayes risk of survival function using Jeffrey's prior under Precautionary loss function is

$R\left[\hat{S}\left(t_{i}\right)\right]_{B P}=2\left\{\left(\sqrt{E\left[\left\{\exp \left[-\left(\frac{t_{i}^{\alpha}}{\beta}\right)\right]\right\}^{2}\right]}\right)-\left(E\left\{\exp \left[-\left(\frac{t_{i}^{\alpha}}{\beta}\right)\right]\right\}\right)\right\}=2\left\{\left\{\sqrt{\frac{\int S\left[\exp \left(-\left(\frac{t_{i}{ }^{\alpha}}{\beta}\right)\right)\right]^{2} \pi_{1}(\alpha, \beta) d \beta d \alpha}{\int \pi_{1}(\alpha, \beta) d \beta d \alpha}}\right\}-\left(\frac{\iint \exp \left[-\left(\frac{t_{i}{ }^{\alpha}}{\beta}\right)\right] \pi_{1}(\alpha, \beta) d \beta d \alpha}{\int \pi_{1}(\alpha, \beta) d \beta d \alpha}\right)\right\}$

Let $u=\left\{\exp \left[-\left(\frac{t_{i}{ }^{\alpha}}{\beta}\right)\right]\right\}^{2}$ and $e=-\left(\frac{t_{i}{ }^{\alpha}}{\beta}\right)$

$u_{1}=\frac{d u}{d \beta}=\frac{-2 u e}{\beta} ; u_{11}=\frac{d u}{d \beta^{2}}=\frac{4 u e}{\beta^{2}}(e+1) ; u_{2}=\frac{d u}{d \alpha}=2 u e\left(\log t_{i}\right) ; u_{22}=\frac{d u}{d \alpha^{2}}=2 u e\left(\log t_{i}\right)^{2}(1+2 e)$

The procedure of Lindley's approximation used in IV (A) is also used to obtain the Bayes risk of survival function using Jeffrey's prior under Precautionary loss function $R\left[\hat{S}\left(t_{i}\right)\right]_{B P}$ is

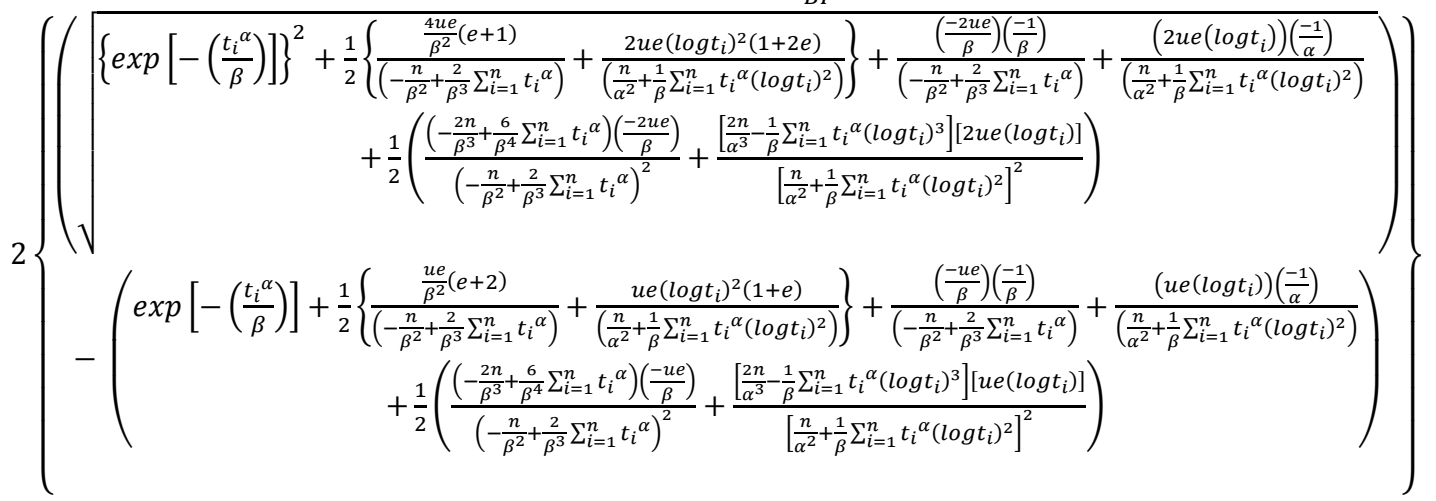

C.Extension of Jeffrey's prior under Linex loss function

The Bayes risk of survival function using extended Jeffrey's prior under Linex loss function is

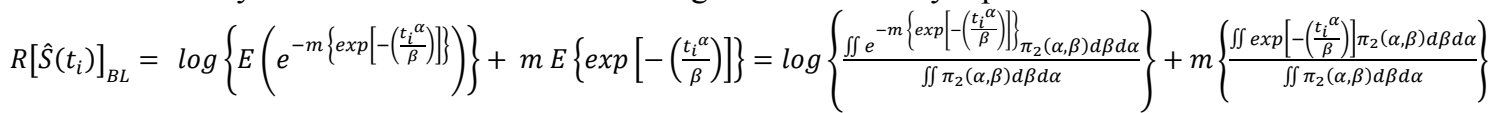

$\rho(\alpha, \beta)=-\log \left(\alpha^{2 p}\right)-\log \left(\beta^{2 p}\right) ; \rho_{1}=\frac{d u}{d \beta}=\frac{-1}{\beta^{2 p}} ; \quad \rho_{2}=\frac{d u}{d \alpha}=-\frac{1}{\alpha^{2 p}}$

The procedure of Lindley's approximation used in IV (A) is also used to obtain the Bayes risk of survival function using extended Jeffrey's prior under Linex loss function $R\left[\hat{S}\left(t_{i}\right)\right]_{B L}$ is 


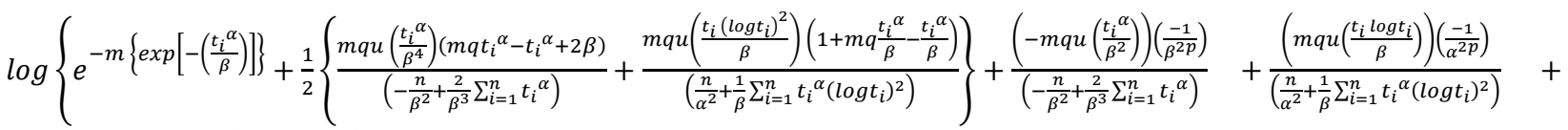

$$
\begin{aligned}
& \left.\frac{1}{2}\left(\frac{\left(-\frac{2 n}{\beta^{3}}+\frac{6}{\beta^{4}} \sum_{i=1}^{n} t_{i}{ }^{\alpha}\right)\left(-m q u\left(\frac{t_{i}{ }^{\alpha}}{\beta^{2}}\right)\right)}{\left(-\frac{n}{\beta^{2}}+\frac{2}{\beta^{3}} \sum_{i=1}^{n} t_{i}^{\alpha}\right)^{2}}+\frac{\left[\frac{2 n}{\alpha^{3}}-\frac{1}{\beta} \sum_{i=1}^{n} t_{i}^{\alpha}\left(\log t_{i}\right)^{3}\right]\left[m q u\left(\frac{t_{i} \log t_{i}}{\beta}\right)\right]}{\left[\frac{n}{\alpha^{2}}+\frac{1}{\beta} \sum_{i=1}^{n} t_{i}{ }^{\alpha}\left(\log t_{i}\right)^{2}\right]^{2}}\right)\right\}+m\left\{\exp \left[-\left(\frac{t_{i}{ }^{\alpha}}{\beta}\right)\right]+\frac{1}{2}\left\{\frac{\frac{u e}{\beta^{2}}(e+2)}{\left(-\frac{n}{\beta^{2}}+\frac{2}{\beta^{3}} \sum_{i=1}^{n} t_{i}^{\alpha}\right)}+\frac{2 u e\left(\log t_{i}\right)^{2}(1+e)}{\left(\frac{n}{\alpha^{2}}+\frac{1}{\beta} \sum_{i=1}^{n} t_{i}{ }^{\alpha}\left(\log t_{i}\right)^{2}\right)}\right\}+\right. \\
& \left.\frac{\left(\frac{u e}{\beta}\right)\left(\frac{-1}{\beta^{2 p}}\right)}{\left(-\frac{n}{\beta^{2}}+\frac{2}{\beta^{3}} \sum_{i=1}^{n} t_{i}^{\alpha}\right)}+\frac{\left(u e\left(\log t_{i}\right)\right)\left(\frac{-1}{\alpha^{2} p}\right)}{\left(\frac{n}{\alpha^{2}}+\frac{1}{\beta} \sum_{i=1}^{n} t_{i}{ }^{\alpha}\left(\log t_{i}\right)^{2}\right)}+\frac{1}{2}\left(\frac{\left(-\frac{2 n}{\beta^{3}}+\frac{6}{\beta^{4}} \sum_{i=1}^{n} t_{i}{ }^{\alpha}\right)\left(\frac{u e}{\beta}\right)}{\left(-\frac{n}{\beta^{2}}+\frac{2}{\beta^{3}} \sum_{i=1}^{n} t_{i}\right)^{2}}+\frac{\left[\frac{2 n}{\alpha^{3}}-\frac{1}{\beta} \sum_{i=1}^{n} t_{i}{ }^{\alpha}\left(\log t_{i}\right)^{3}\right]\left[u e\left(\log t_{i}\right)\right]}{\left[\frac{n}{\alpha^{2}}+\frac{1}{\beta} \sum_{i=1}^{n} t_{i}{ }^{\alpha}\left(\log t_{i}\right)^{2}\right]^{2}}\right)\right\}
\end{aligned}
$$

\section{Lognormal-Inverted Gamma prior under Linex loss function}

The Bayes risk of survival function using Lognormal-Inverted Gamma prior under Linex loss function is

$$
\begin{aligned}
& R\left[\hat{S}\left(t_{i}\right)\right]_{B L}=\log \left\{E\left(e^{-m\left\{\exp \left[-\left(\frac{t_{i}^{\alpha}}{\beta}\right)\right]\right\}}\right)\right\}+m E\left\{\exp \left[-\left(\frac{t_{i}^{\alpha}}{\beta}\right)\right]\right\}=\log \left\{\frac{\int S e^{-m\left\{\exp \left[-\left(\frac{t_{i}^{\alpha}}{\beta}\right)\right]\right\}_{\pi_{3}(\alpha, \beta) d \beta d \alpha}}}{\iint \pi_{3}(\alpha, \beta) d \beta d \alpha}\right\}+m\left\{\frac{\iint \exp \left[-\left(\frac{t_{i}^{\alpha}}{\beta}\right)\right] \pi_{3}(\alpha, \beta) d \beta d \alpha}{\iint \pi_{3}(\alpha, \beta) d \beta d \alpha}\right\} \\
& \rho(\alpha, \beta)=-\log \alpha-\frac{(\log \alpha)^{2}}{2 c^{2}}-(a+1) \log \beta-\frac{b}{\beta} ; \rho_{1}=\frac{d u}{d \beta}=\frac{-(a+1)}{\beta}+\frac{b}{\beta^{2}} ; \rho_{2}=\frac{d u}{d \alpha}=-\frac{1}{\alpha}-\frac{\log \alpha}{\alpha c^{2}}
\end{aligned}
$$

The procedure of Lindley's approximation used in IV (A) is also used to obtain the Bayes risk of survival function using Lognormal-Inverted Gamma prior under Linex loss function $R\left[\hat{S}\left(t_{i}\right)\right]_{B L}$ is

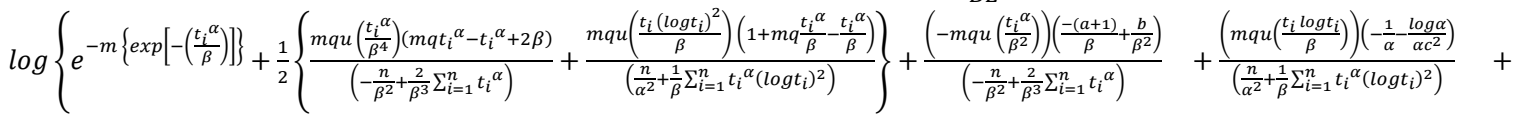

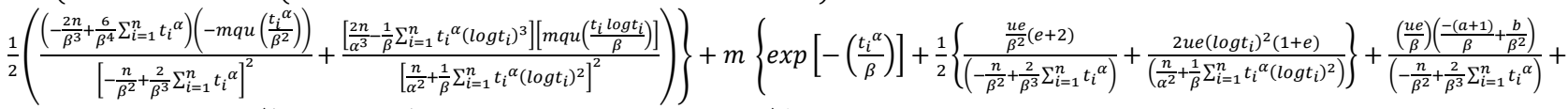

$$
\begin{aligned}
& \left.\frac{\left(u e\left(\log _{i}\right)\right)\left(-\frac{1}{\alpha}-\frac{\log \alpha}{\alpha c^{2}}\right)}{\left(\frac{n}{\alpha^{2}}+\frac{1}{\beta} \sum_{i=1}^{n} t_{i}^{\alpha}\left(\log _{i}\right)^{2}\right)}+\frac{1}{2}\left(\frac{\left(-\frac{2 n}{\beta^{3}}+\frac{6}{\beta^{4}} \sum_{i=1}^{n} t_{i}{ }^{\alpha}\right)\left(\frac{u e}{\beta}\right)}{\left[-\frac{n}{\beta^{2}}+\frac{2}{\beta^{3}} \sum_{i=1}^{n} t_{i}^{\alpha}\right]^{2}}+\frac{\left[\frac{2 n}{\alpha^{3}}-\frac{1}{\beta} \sum_{i=1}^{n} t_{i}^{\alpha}\left(\log _{i}\right)^{3}\right]\left[u e\left(\log t_{i}\right)\right]}{\left[\frac{n}{\alpha^{2}}+\frac{1}{\beta} \sum_{i=1}^{n} t_{i}{ }^{\alpha}\left(\log _{i}\right)^{2}\right]^{2}}\right)\right\}
\end{aligned}
$$

\section{E.Gamma-Gamma prior under Squared Error loss function}

The Bayes risk of survival function using Gamma-Gamma prior under Squared error loss function is

$R\left[\hat{S}\left(t_{i}\right)\right]_{B S}=E\left\{\left\{\exp \left[-\left(\frac{t_{i}{ }^{\alpha}}{\beta}\right)\right]\right\}^{2}\right\}-\left[E\left\{\exp \left[-\left(\frac{t_{i} \alpha}{\beta}\right)\right]\right\}\right]^{2}=\frac{\iint\left[\exp \left(-\left(\frac{t_{i}{ }^{\alpha}}{\beta}\right)\right)\right]^{2} \pi_{4}(\alpha, \beta) d \beta d \alpha}{\iint \pi_{4}(\alpha, \beta) d \beta d \alpha}-\left\{\frac{\iint \exp \left[-\left(\frac{t_{i}{ }^{\alpha}}{\beta}\right)\right] \pi_{4}(\alpha, \beta) d \beta d \alpha}{\iint \pi_{4}(\alpha, \beta) d \beta d \alpha}\right\}^{2}$

$\rho(\alpha, \beta)=\left(a_{1}-1\right) \log \alpha-b_{1} \alpha+\left(d_{1}-1\right) \log \beta-c_{1} \beta ; \rho_{1}=\frac{d u}{d \beta}=\frac{d_{1}-1}{\beta}-c_{1} ; \quad \rho_{2}=\frac{d u}{d \alpha}=\frac{a_{1}-1}{\alpha}-b_{1}$

The procedure of Lindley' approximation used in IV (A) is also used to obtain the Bayes risk of survival function using

Gamma-Gamma prior under squared error loss function $R\left[\hat{S}\left(t_{i}\right)\right]_{B S}$ is

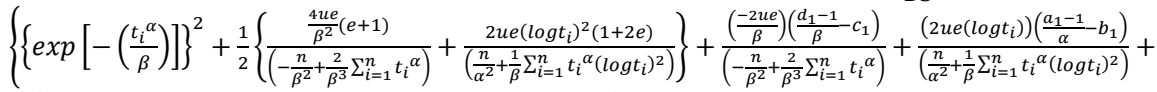

$\left.\frac{1}{2}\left(\frac{\left(-\frac{2 n}{\beta^{3}}+\frac{6}{\beta^{4}} \sum_{i=1}^{n} t_{i}^{\alpha}\right)\left(\frac{-2 u e}{\beta}\right)}{\left(-\frac{n}{\beta^{2}}+\frac{2}{\beta^{3}} \sum_{i=1}^{n} t_{i}^{\alpha}\right)^{2}}+\frac{\left[\frac{2 n}{\alpha^{3}} \frac{1}{\beta} \sum_{i=1}^{n} t_{i}{ }^{\alpha}\left(\log t_{i}\right)^{3}\right]\left[2 u e\left(\log t_{i}\right)\right]}{\left[\frac{n}{\alpha^{2}}+\frac{1}{\beta} \sum_{i=1}^{n} t_{i}^{\alpha}\left(\log _{i}\right)^{2}\right]^{2}}\right)\right\}-$

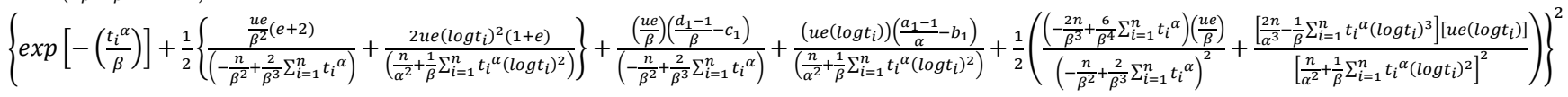

\section{F.Gamma-Gamma prior under Linex loss function}

The Bayes risk of survival function using Gamma-Gamma prior under Linex loss function is

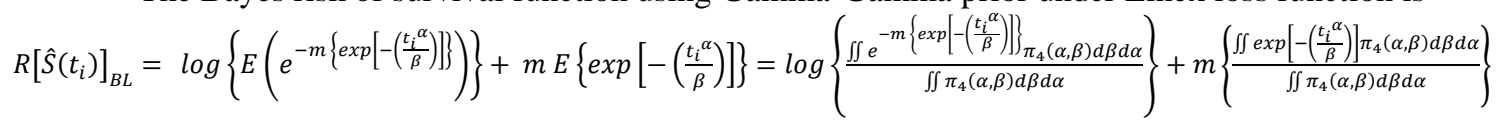

The procedure of Lindley's approximation used in IV (A) is also used to obtain the Bayes risk of survival function using Gamma-Gamma prior under Linex loss function $R\left[\hat{S}\left(t_{i}\right)\right]_{B L}$ is 


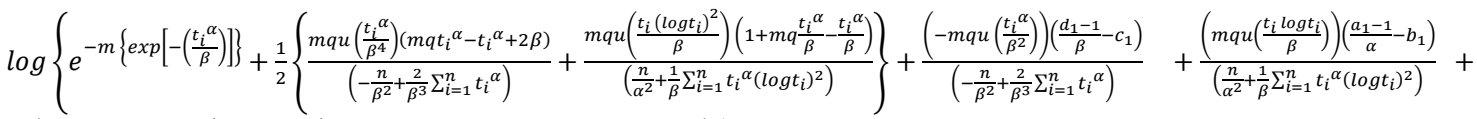

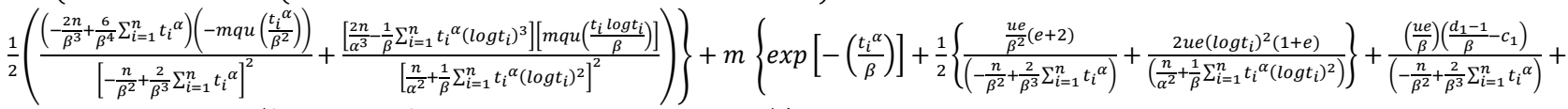

$$
\begin{aligned}
& \left.\frac{\left(u e\left(\log _{i}\right)\right)\left(\frac{a_{1}-1}{\alpha}-b_{1}\right)}{\left(\frac{n}{\alpha^{2}}+\frac{1}{\beta} \sum_{i=1}^{n} t_{i}^{\alpha}\left(\log _{i}\right)^{2}\right)}+\frac{1}{2}\left(\frac{\left(-\frac{2 n}{\beta^{3}}+\frac{6}{\beta^{4}} \sum_{i=1}^{n} t_{i}{ }^{\alpha}\right)\left(\frac{u e}{\beta}\right)}{\left[-\frac{n}{\beta^{2}}+\frac{2}{\beta^{3}} \sum_{i=1}^{n} t_{i}^{\alpha}\right]^{2}}+\frac{\left[\frac{2 n}{\alpha^{3}}-\frac{1}{\beta} \sum_{i=1}^{n} t_{i}^{\alpha}\left(\log _{i}\right)^{3}\right]\left[u e\left(\log t_{i}\right)\right]}{\left[\frac{n}{\alpha^{2}}+\frac{1}{\beta} \sum_{i=1}^{n} t_{i}^{\alpha}\left(\log _{i}\right)^{2}\right]^{2}}\right)\right\}
\end{aligned}
$$

\section{G.Gamma-Gamma prior under General Entropy loss function}

The Bayes risk of survival function using Gamma-Gamma prior under General entropy loss function is

$R\left[\hat{S}\left(t_{i}\right)\right]_{B G}=k\left(E\left\{\log \left\{\exp \left[-\left(\frac{t_{i}{ }^{\alpha}}{\beta}\right)\right]\right\}\right\}\right)+\log \left(E\left\{\left[\exp \left(-\left(\frac{t_{i}{ }^{\alpha}}{\beta}\right)\right)\right]^{-k}\right\}\right)=k\left[\frac{\int \log \left\{\exp \left[-\left(\frac{t_{i}^{\alpha}}{\beta}\right)\right] \pi_{\pi_{4}}(\alpha, \beta) d \beta d \alpha\right.}{\iint \pi_{4}(\alpha, \beta) d \beta d \alpha}\right]+\log \left\{\frac{\iint\left\{\exp \left[-\left(-\frac{t_{i}{ }^{\alpha}}{\beta}\right)\right]\right\}^{-k} \pi_{4}(\alpha, \beta) d \beta d \alpha}{\iint \pi_{4}(\alpha, \beta) d \beta d \alpha}\right\}$

Let $u=\left[-\left(\frac{t_{i}{ }^{\alpha}}{\beta}\right)\right] ; u_{1}=\frac{d u}{d \beta}=\frac{-u}{\beta} ; u_{11}=\frac{d u}{d \beta^{2}}=\frac{2 u}{\beta^{2}} ; u_{2}=\frac{d u}{d \alpha}=u\left(\log t_{i}\right) ; u_{22}=\frac{d u}{d \alpha^{2}}=u\left(\log t_{i}\right)^{2}$

Let $u=\left[\exp \left(-\left(\frac{t_{i}{ }^{\alpha}}{\beta}\right)\right)\right]^{-k} ; u_{1}=\frac{d u}{d \beta}=\frac{k u e}{\beta} ; u_{11}=\frac{d^{2} u}{d \beta^{2}}=\frac{k u e}{\beta^{2}}(k e-2) ; u_{2}=\frac{d u}{d \alpha}=-k u e\left(\log _{i}\right) ; u_{22}=\frac{d^{2} u}{d \alpha^{2}}=k u e\left(\log _{i}\right)^{2}(k e-1)$

The procedure of Lindley' approximation used in IV (A) is also used to obtain the Bayes risk of survival function using Gamma-Gamma prior under General entropy loss function $R\left[\hat{S}\left(t_{i}\right)\right]_{B G}$ is

$\left\{\left[-\left(\frac{t_{i}{ }^{\alpha}}{\beta}\right)\right]+\frac{1}{2}\left\{\frac{\frac{2 u}{\beta^{2}}}{\left(-\frac{n}{\beta^{2}}+\frac{2}{\beta^{3}} \sum_{i=1}^{n} t_{i}{ }^{\alpha}\right)}+\frac{u\left(\log _{t_{i}}{ }^{2}\right.}{\left(\frac{n}{\alpha^{2}}+\frac{1}{\beta} \sum_{i=1}^{n} t_{i}{ }^{\alpha}\left(\log _{i}\right)^{2}\right)}\right\}+\frac{\left(\frac{-u}{\beta}\right)\left(\frac{d_{1}-1}{\beta} c_{1}\right)}{\left(-\frac{n}{\beta^{2}}+\frac{2}{\beta^{3}} \sum_{i=1}^{n} t_{i}{ }^{\alpha}\right)}+\frac{u\left(\log t_{i}\right)\left(\frac{a_{1}-1}{\alpha}-b_{1}\right)}{\left(\frac{n}{\alpha^{2}}+\frac{1}{\beta} \sum_{i=1}^{n} t_{i}{ }^{\alpha}\left(\log _{i}\right)^{2}\right)}+\right.$ $\left.\frac{1}{2}\left(\frac{\left[-\frac{2 n}{\beta^{3}}+\frac{6}{\beta^{4}} \sum_{i=1}^{n} t_{i}{ }^{\alpha}\right]\left(\frac{-u}{\beta}\right)}{\left(-\frac{n}{\beta^{2}}+\frac{2}{\beta^{3}} \sum_{i=1}^{n} t_{i}\right)^{2}}+\frac{\left[\frac{2 n}{\alpha^{3}}-\frac{1}{\beta} \sum_{i=1}^{n} t_{i}{ }^{\alpha}\left(\log _{t}\right)^{3}\right]\left[u\left(\log t_{i}\right)\right]}{\left[\frac{n}{\alpha^{2}}+\frac{1}{\beta} \sum_{i=1}^{n} t_{i}{ }^{\alpha}\left(\log t_{i}\right)^{2}\right]^{2}}\right)\right\}+$

$\log \left\{\left\{\exp \left[-\left(\frac{t_{i}^{\alpha}}{\beta}\right)\right]\right\}^{-k}+\frac{1}{2}\left\{\frac{\frac{k u e}{\beta^{2}}(k e-2)}{\left(-\frac{n}{\beta^{2}}+\frac{2}{\beta^{3}} \sum_{i=1}^{n} t_{i}{ }^{\alpha}\right)}+\frac{k u e\left(\log t_{i}\right)^{2}(k e-1)}{\left(\frac{n}{\alpha^{2}}+\frac{1}{\beta} \sum_{i=1}^{n} t_{i}^{\alpha}\left(\log _{i}\right)^{2}\right)}\right\}+\frac{\left(\frac{k u e}{\beta}\right)\left(\frac{d_{1}-1}{\beta}-c_{1}\right)}{\left(-\frac{n}{\beta^{2}}+\frac{2}{\beta^{3}} \sum_{i=1}^{n} t_{i}{ }^{\alpha}\right)}+\frac{\left(-k u e\left(\log t_{i}\right)\right)\left(\frac{a_{1}-1}{\alpha}-b_{1}\right)}{\left(\frac{n}{\alpha^{2}}+\frac{1}{\beta} \sum_{i=1}^{n} t_{i}{ }^{\alpha}\left(\log t_{i}\right)^{2}\right)}+\frac{1}{2}\left(\frac{-\frac{2 n}{\beta^{3}}+\frac{6}{\beta^{4}} \sum_{i=1}^{n} t_{i}{ }^{\alpha}\left(\frac{k u e}{\beta}\right)}{\left(-\frac{n}{\beta^{2}}+\frac{2}{\beta^{3}} \sum_{i=1}^{n} t_{i}{ }^{\alpha}\right)^{2}}+\right.\right.$ $\left.\left.\frac{\left[\frac{2 n}{\alpha^{3}}-\frac{1}{\beta} \sum_{i=1}^{n} t_{i}{ }^{\alpha}\left(\log t_{i}\right)^{3}\right]\left[-k u e\left(\log t_{i}\right)\right]}{\left[\frac{n}{\alpha^{2}}+\frac{1}{\beta} \sum_{i=1}^{n} t_{i}{ }^{\alpha}\left(\log _{i}\right)^{2}\right]^{2}}\right)\right\}$

\section{H.Gamma-Gamma prior under Quadratic loss function}

The Bayes risk of survival function using Gamma-Gamma prior under Quadratic loss function is

$$
R\left[\hat{S}\left(t_{i}\right)\right]_{B Q}=1-\frac{\left\{E\left\{\left[\exp \left(-\left(\frac{t_{i}{ }^{\alpha}}{\beta}\right)\right)\right]^{-1}\right\}\right\}^{2}}{E\left\{\left[\exp \left(-\left(\frac{t_{i}{ }^{\alpha}}{\beta}\right)\right)\right]^{-2}\right\}}=1-\frac{\left\{\frac{\left\{\left[\exp \left(-\left(\frac{t_{i}{ }^{\alpha}}{\beta}\right)\right)\right]^{-1} \pi_{4}(\alpha, \beta) d \beta d \alpha\right.}{\iint \pi_{4}(\alpha, \beta) d \beta d \alpha}\right\}^{2}}{\left\{\int\left[\frac{\left.\exp \left(-\left(\frac{t_{i}{ }^{\alpha}}{\beta}\right)\right)\right]^{-2} \pi_{4}(\alpha, \beta) d \beta d \alpha}{\iint \pi_{4}(\alpha, \beta) d \beta d \alpha}\right\}\right.}
$$

Let $u=\left[\exp \left(-\left(\frac{t_{i}{ }^{\alpha}}{\beta}\right)\right)\right]^{-1}$ and $\mathrm{e}=-\left(\frac{t_{i}{ }^{\alpha}}{\beta}\right)$

$u_{1}=\frac{d u}{d \beta}=\frac{u e}{\beta} ; u_{11}=\frac{d^{2} u}{d \beta^{2}}=\frac{u e}{\beta^{2}}(e-2) ; u_{2}=\frac{d u}{d \alpha}=-u e\left(\log t_{i}\right) ; u_{22}=\frac{d^{2} u}{d \alpha^{2}}=-u e\left(\log _{i}\right)^{2}(1-e)$

Let $u=\left[\exp \left(-\left(\frac{t_{i} \alpha}{\beta}\right)\right)\right]^{-2} ; u_{1}=\frac{d u}{d \beta}=\frac{2 u e}{\beta} ; u_{11}=\frac{d^{2} u}{d \beta^{2}}=\frac{4 u e}{\beta^{2}}(e-1) ; u_{2}=\frac{d u}{d \alpha}=-2 u e\left(\log t_{i}\right) ; u_{22}=\frac{d^{2} u}{d \alpha^{2}}=2 u e\left(\log t_{i}\right)^{2}(2 e-1)$

The procedure of Lindley' approximation used in IV (A) is also used to obtain the Bayes risk of survival function using Gamma-Gamma prior under Quadratic loss function $R\left[\hat{S}\left(t_{i}\right)\right]_{B Q}$ is

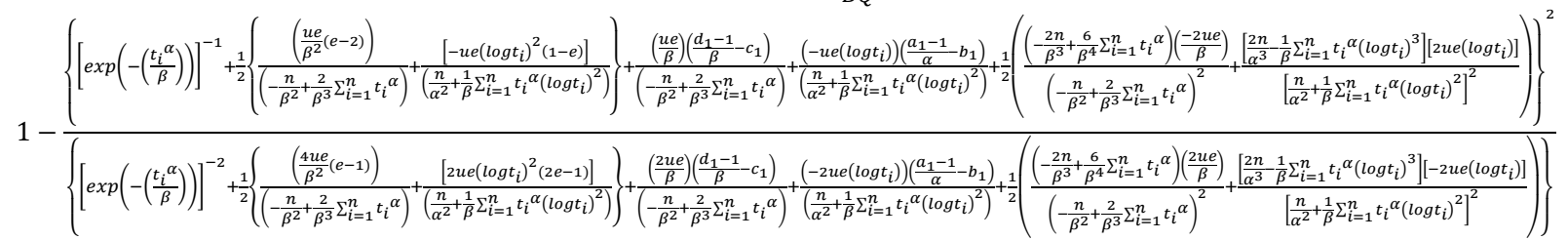




\section{I.Gamma-Gamma prior under Weighted loss function}

The Bayes risk of survival function using Gamma-Gamma prior under Weighted loss function is

$R\left[\hat{S}\left(t_{i}\right)\right]_{B W}=E\left\{\exp \left[-\left(\frac{t_{i}{ }^{\alpha}}{\beta}\right)\right]\right\}-\left[E\left\{\left[\exp \left(-\left(\frac{t_{i}{ }^{\alpha}}{\beta}\right)\right)\right]^{-1}\right\}\right]^{-1}=\frac{\iint \exp \left[-\left(\frac{t_{i}{ }^{\alpha}}{\beta}\right)\right] \pi_{4}(\alpha, \beta) d \beta d \alpha}{\iint \pi_{4}(\alpha, \beta) d \beta d \alpha}-\left[\frac{\iint\left[\exp \left(-\left(\frac{t_{i}{ }^{\alpha}}{\beta}\right)\right)\right]^{-1} \pi_{4}(\alpha, \beta) d \beta d \alpha}{\iint \pi_{4}(\alpha, \beta) d \beta d \alpha}\right]^{-1}$

The procedure of Lindley' approximation used in IV (A) is also used to obtain the Bayes risk of survival function using Gamma-Gamma prior under Weighted loss function $R\left[\hat{S}\left(t_{i}\right)\right]_{B W}$ is

$\left\{\exp \left[-\left(\frac{t_{i}{ }^{\alpha}}{\beta}\right)\right]+\frac{1}{2}\left\{\frac{\frac{u e}{\beta^{2}}(e+2)}{\left(-\frac{n}{\beta^{2}}+\frac{2}{\beta^{3}} \sum_{i=1}^{n} t_{i}{ }^{\alpha}\right)}+\frac{2 u e\left(\log t_{i}\right)^{2}(1+e)}{\left(\frac{n}{\alpha^{2}}+\frac{1}{\beta} \sum_{i=1}^{n} t_{i}{ }^{\alpha}\left(\log t_{i}\right)^{2}\right)}\right\}+\frac{\left(\frac{u e}{\beta}\right)\left(\frac{d_{1}-1}{\beta} c_{1}\right)}{\left(-\frac{n}{\beta^{2}}+\frac{2}{\beta^{3}} \sum_{i=1}^{n} t_{i}{ }^{\alpha}\right)}+\frac{\left(u e\left(\log t_{i}\right)\right)\left(\frac{a_{1}-1}{\alpha}-b_{1}\right)}{\left(\frac{n}{\alpha^{2}}+\frac{1}{\beta} \sum_{i=1}^{n} t_{i}{ }^{\alpha}\left(\log _{i}\right)^{2}\right)}+\frac{1}{2}\left(\frac{\left(-\frac{2 n}{\beta^{3}}+\frac{6}{\beta^{4}} \sum_{i=1}^{n} t_{i}{ }^{\alpha}\right)\left(\frac{u e}{\beta}\right)}{\left(-\frac{n}{\beta^{2}}+\frac{2}{\beta^{3}} \sum_{i=1}^{n} t_{i}\right)^{2}}+\frac{\left[\frac{2 n}{\alpha^{3}}-\frac{1}{\beta} \sum_{i=1}^{n} t_{i}^{\alpha}\left(\log t_{i}\right)^{3}\right]\left[u e\left(\log t_{i}\right)\right]}{\left(\frac{n}{\alpha^{2}}+\frac{1}{\beta} \sum_{i=1}^{n} t_{i}{ }^{\alpha}\left(\log t_{i}\right)^{2}\right)^{2}}\right)\right\}-$

$\left\{\left\{\exp \left[-\left(\frac{t_{i}^{\alpha}}{\beta}\right)\right]\right\}^{-1}+\frac{1}{2}\left\{\frac{\frac{u e}{\beta^{2}}(e-2)}{\left(-\frac{n}{\beta^{2}}+\frac{2}{\beta^{3}} \sum_{i=1}^{n} t_{i}{ }^{\alpha}\right)}+\frac{\left[-u e\left(\log t_{i}\right)^{2}(1-e)\right]}{\left(\frac{n}{\alpha^{2}}+\frac{1}{\beta} \sum_{i=1}^{n} t_{i}{ }^{\alpha}\left(\log t_{i}\right)^{2}\right)}\right\}+\frac{\left(\frac{u e}{\beta}\right)\left(\frac{d_{1}-1}{\beta}-c_{1}\right)}{\left(-\frac{n}{\beta^{2}}+\frac{2}{\beta^{3}} \sum_{i=1}^{n} t_{i}{ }^{\alpha}\right)}+\frac{\left(-u e\left(\log t_{i}\right)\right)\left(\frac{a_{1}-1}{\alpha}-b_{1}\right)}{\left(\frac{n}{\alpha^{2}}+\frac{1}{\beta} \sum_{i=1}^{n} t_{i}{ }^{\alpha}\left(\log _{i}\right)^{2}\right)}+\right.$

$\left.\frac{1}{2}\left(\frac{\left(-\frac{2 n}{\beta^{3}}+\frac{6}{\beta^{4}} \sum_{i=1}^{n} t_{i}{ }^{\alpha}\right)\left(\frac{u e}{\beta}\right)}{\left(-\frac{n}{\beta^{2}}+\frac{2}{\beta^{3}} \sum_{i=1}^{n} t_{i}^{\alpha}\right)^{2}}+\frac{\left[\frac{2 n}{\alpha^{3}}-\frac{1}{\beta} \sum_{i=1}^{n} t_{i}{ }^{\alpha}\left(\log t_{i}\right)^{3}\right]\left[-u e\left(\log t_{i}\right)\right]}{\left(\frac{n}{\alpha^{2}}+\frac{1}{\beta} \sum_{i=1}^{n} t_{i}{ }^{\alpha}\left(\log _{i}\right)^{2}\right)^{2}}\right)\right\}^{-1}$

\section{J.Gamma-Gamma prior under Precautionary loss function}

The Bayes risk of survival function using Gamma-Gamma prior under Precautionary loss function is

$R\left[\hat{S}\left(t_{i}\right)\right]_{B P}=2\left\{\left(\sqrt{E\left\{\left\{\exp \left[-\left(\frac{t_{i}{ }^{\alpha}}{\beta}\right)\right]\right\}^{2}\right\}}\right)-\left(E\left\{\exp \left[-\left(\frac{t_{i}{ }^{\alpha}}{\beta}\right)\right]\right\}\right)\right\}=2\left\{\left\{\sqrt{\frac{\iint\left[\exp \left(-\left(\frac{t_{i}{ }^{\alpha}}{\beta}\right)\right)\right]^{2} \pi_{1}(\alpha, \beta) d \beta d \alpha}{\iint \pi_{1}(\alpha, \beta) d \beta d \alpha}}\right\}-\left(\frac{\iint \exp \left[-\left(\frac{t_{i}{ }^{\alpha}}{\beta}\right)\right] \pi_{1}(\alpha, \beta) d \beta d \alpha}{\iint \pi_{1}(\alpha, \beta) d \beta d \alpha}\right)\right\}$

Let $u=\left\{\exp \left[-\left(\frac{t_{i}{ }^{\alpha}}{\beta}\right)\right]\right\}^{2}$ and $e=-\left(\frac{t_{i}{ }^{\alpha}}{\beta}\right)$

$$
u_{1}=\frac{d u}{d \beta}=\frac{-2 u e}{\beta} ; u_{11}=\frac{d u}{d \beta^{2}}=\frac{4 u e}{\beta^{2}}(e+1) ; u_{2}=\frac{d u}{d \alpha}=2 u e\left(\log t_{i}\right) ; u_{22}=\frac{d u}{d \alpha^{2}}=2 u e\left(\log t_{i}\right)^{2}(1+2 e)
$$

The procedure of Lindley's approximation used in IV (A) is also used to obtain the Bayes risk of survival function using Gamma-Gamma prior under Precautionary loss function $R\left[\hat{S}\left(t_{i}\right)\right]_{B P}$ is

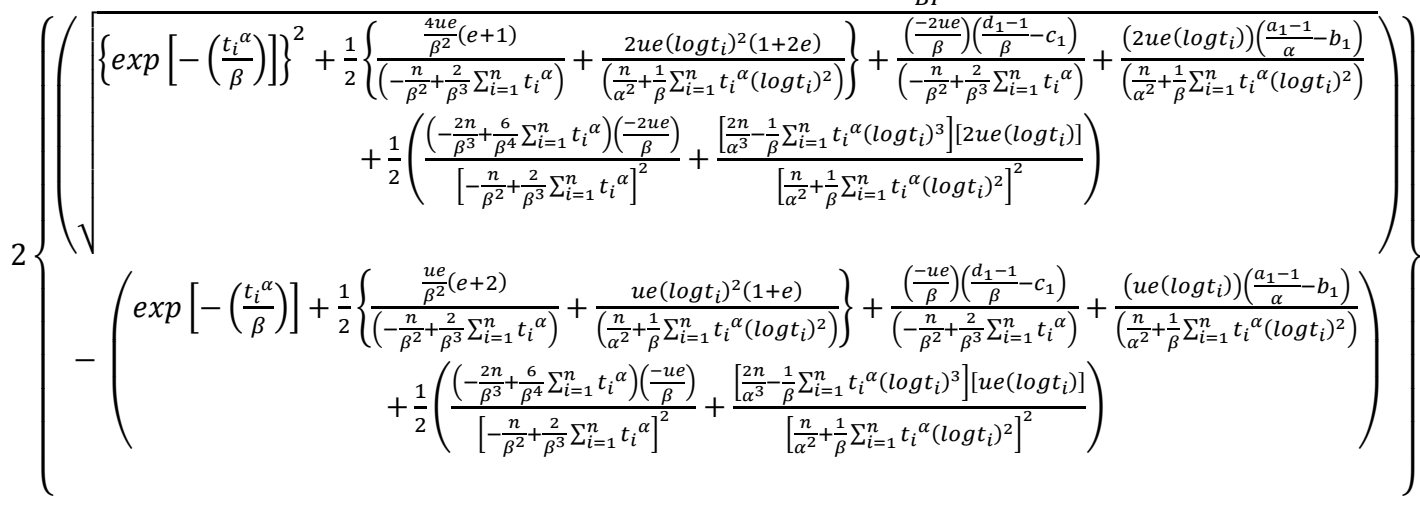

K.Gamma-Gamma prior under Squared logarithmic loss function

The Bayes risk of survival function using Exponential-Gamma prior under Squared logarithmic loss function is

$\left.R\left[\hat{S}\left(t_{i}\right)\right]_{B S L}=E\left\{\left\{\log \left\{\exp \left[-\left(\frac{t_{i}{ }^{\alpha}}{\beta}\right)\right]\right\}\right\}\right\}^{2}\right\}-\left\{E\left\{\log \left\{\exp \left[-\left(\frac{t_{i}{ }^{\alpha}}{\beta}\right)\right]\right\}\right\}\right\}^{2}=\frac{\iint\left\{\log \left[\exp \left(-\left(\frac{t_{i}{ }^{\alpha}}{\beta}\right)\right)\right]\right\}^{2} \pi_{4}(\alpha, \beta) d \beta d \alpha}{\iint \pi_{4}(\alpha, \beta) d \beta d \alpha}-\left\{\int \log \left\{\exp \left[-\left(\frac{t_{i}{ }^{\alpha}}{\beta}\right)\right]\right\}_{\pi_{4}(\alpha, \beta) d \beta d \alpha}\right\}^{2}$

Let $u=-\left(\frac{t_{i}^{\alpha}}{\beta}\right) ; u_{1}=\frac{d u}{d \beta}=\frac{-u}{\beta} \quad ; u_{11}=\frac{d u}{d \beta^{2}}=\frac{2 u}{\beta^{2}} ; u_{2}=\frac{d u}{d \alpha}=u\left(\log t_{i}\right) \quad ; u_{22}=\frac{d u}{d \alpha^{2}}=u\left(\log t_{i}\right)^{2}$

Let $u=\left[-\left(\frac{t_{i}{ }^{\alpha}}{\beta}\right)\right]^{2} ; u_{1}=\frac{d u}{d \beta}=\frac{-2 u}{\beta} ; u_{11}=\frac{d u}{d \beta^{2}}=\frac{6 u}{\beta^{2}} ; u_{2}=\frac{d u}{d \alpha}=2 u\left(\log t_{i}\right) \quad ; u_{22}=\frac{d u}{d \alpha^{2}}=4 u\left(\log t_{i}\right)^{2}$

The procedure of Lindley' approximation used in IV(A) is also used to obtain the Bayes risk of survival function using Gamma-Gamma prior under Squared logarithmic loss function $R\left[\hat{S}\left(t_{i}\right)\right]_{B S L}$ is

$\left\{\left[-\left(\frac{t_{i}{ }^{\alpha}}{\beta}\right)\right]^{2}+\frac{1}{2}\left\{\frac{\left(\frac{6 u}{\beta^{2}}\right)}{\left(\frac{-n}{\beta^{2}}+\frac{2}{\beta^{3}} \sum_{i=1}^{n} t_{i}^{\alpha}\right)}+\frac{4 u\left(\log t_{i}\right)^{2}}{\left(\frac{n}{\alpha^{2}}+\frac{1}{\beta} \sum_{i=1}^{n} t_{i}{ }^{\alpha}\left(\log _{i}\right)^{2}\right)}\right\}+\frac{\left(\frac{-2 u}{\beta}\right)\left(\frac{d_{1}-1}{\beta}-c_{1}\right)}{\left(\frac{-n}{\beta^{2}}+\frac{2}{\beta^{3}} \sum_{i=1}^{n} t_{i}{ }^{\alpha}\right)}+\frac{\left(2 u\left(\log t_{i}\right)\right)\left(\frac{a_{1}-1}{\alpha}-b_{1}\right)}{\left(\frac{n}{\alpha^{2}}+\frac{1}{\beta} \sum_{i=1}^{n} t_{i}{ }^{\alpha}\left(\log _{i}\right)^{2}\right)}+\right.$ $\left.\frac{1}{2}\left(\frac{\left(-\frac{2 n}{\beta^{3}}+\frac{6}{\beta^{4}} \sum_{i=1}^{n} t_{i}^{\alpha}\right)\left(\frac{-2 u}{\beta}\right)}{\left[\frac{-n}{\beta^{2}}+\frac{2}{\beta^{3}} \sum_{i=1}^{n} t_{i}^{\alpha}\right]^{2}}+\frac{\left[\frac{2 n}{\alpha^{3}}-\frac{1}{\beta} \sum_{i=1}^{n} t_{i}^{\alpha}\left(\log t_{i}\right)^{3}\right]\left[2 u\left(\log t_{i}\right)\right]}{\left[\frac{n}{\alpha^{2}}+\frac{1}{\beta} \sum_{i=1}^{n} t_{i}^{\alpha}\left(\log t_{i}\right)^{2}\right]^{2}}\right)\right\}-\left\{\left[-\left(\frac{t_{i}{ }^{\alpha}}{\beta}\right)\right]+\frac{1}{2}\left\{\frac{\left(\frac{2 u}{\beta^{2}}\right)}{\left(\frac{-n}{\beta^{2}}+\frac{2}{\beta^{3}} \sum_{i=1}^{n} t_{i}{ }^{\alpha}\right)}+\frac{u\left(\log t_{i}\right)^{2}}{\left(\frac{n}{\alpha^{2}}+\frac{1}{\beta} \sum_{i=1}^{n} t_{i}{ }^{\alpha}\left(\log t_{i}\right)^{2}\right)}\right\}+\frac{\left(\frac{-u}{\beta}\right)\left(\frac{d_{1}-1}{\beta}-c_{1}\right)}{\left(\frac{-n}{\beta^{2}}+\frac{2}{\beta^{3}} \sum_{i=1}^{n} t_{i}{ }^{\alpha}\right)}+\right.$ 
$\left.\frac{\left(u\left(\log t_{i}\right)\right)\left(\frac{a_{1}-1}{\alpha}-b_{1}\right)}{\left(\frac{n}{\alpha^{2}}+\frac{1}{\beta} \sum_{i=1}^{n} t_{i}^{\alpha}\left(\log _{i}\right)^{2}\right)}+\frac{1}{2}\left(\frac{\left(-\frac{2 n}{\beta^{3}}+\frac{6}{\beta^{4}} \sum_{i=1}^{n} t_{i}{ }^{\alpha}\right)\left(\frac{-u}{\beta}\right)}{\left[\frac{-n}{\beta^{2}}+\frac{2}{\beta^{3}} \sum_{i=1}^{n} t_{i}^{\alpha}\right]^{2}}+\frac{\left[\frac{2 n}{\alpha^{3}}-\frac{1}{\beta} \sum_{i=1}^{n} t_{i}^{\alpha}\left(\log t_{i}\right)^{3}\right]\left[u\left(\log t_{i}\right)\right]}{\left[\frac{n}{\alpha^{2}}+\frac{1}{\beta} \sum_{i=1}^{n} t_{i}^{\alpha}\left(\log _{i}\right)^{2}\right]^{2}}\right)\right\}^{2}$

\section{Simulation STUdY}

In this study, we chose a sample size of $n=25,50$ and 100 to represent small, medium and large dataset. The posterior risk of survival function is estimated for Weibull distribution with two parameter using non-informative and informative priors such as Jeffrey's, Extension of Jeffrey's, Lognormal-Inverted Gamma, Exponential-Gamma and Gamma-Gamma using with Squared error loss function (SELF), Linex loss function (LLF), General entropy loss function (GELF), Quadratic loss function (QLF), Weighted loss function (WLF), Squared logarithmic loss function (SLLF) and Precautionary loss function (PLF). The values of the parameters chosen as $\alpha=0.8,1.2,3$ and $\beta=0.5,1.5,5$. The hyperparameter values of Extension of Jeffrey's prior is $\mathrm{p}=0.4,1.4$ and for the informative priors are chosen as $\mathrm{a}=0.4,1.4 ; \mathrm{b}=0.6,1.6$ and $\mathrm{c}=0.9,1.9 ; \mathrm{a}_{1}=2,4 ; \mathrm{b}_{1}=0.2,0.5 ; \mathrm{c}_{1}=0.8,1.5$ and $\mathrm{d}_{1}=4,3$. The results of the simulation study are obtained, discussed and reported as follows:

The Bayes risk of survival function for the two parameter Weibull distribution using non-informative prior (Jeffrey's prior) under various loss functions is obtained and presented in Table-I.

Table I: Estimated Value of Bayes Risk of Survival Function Under Jeffrey's Prior

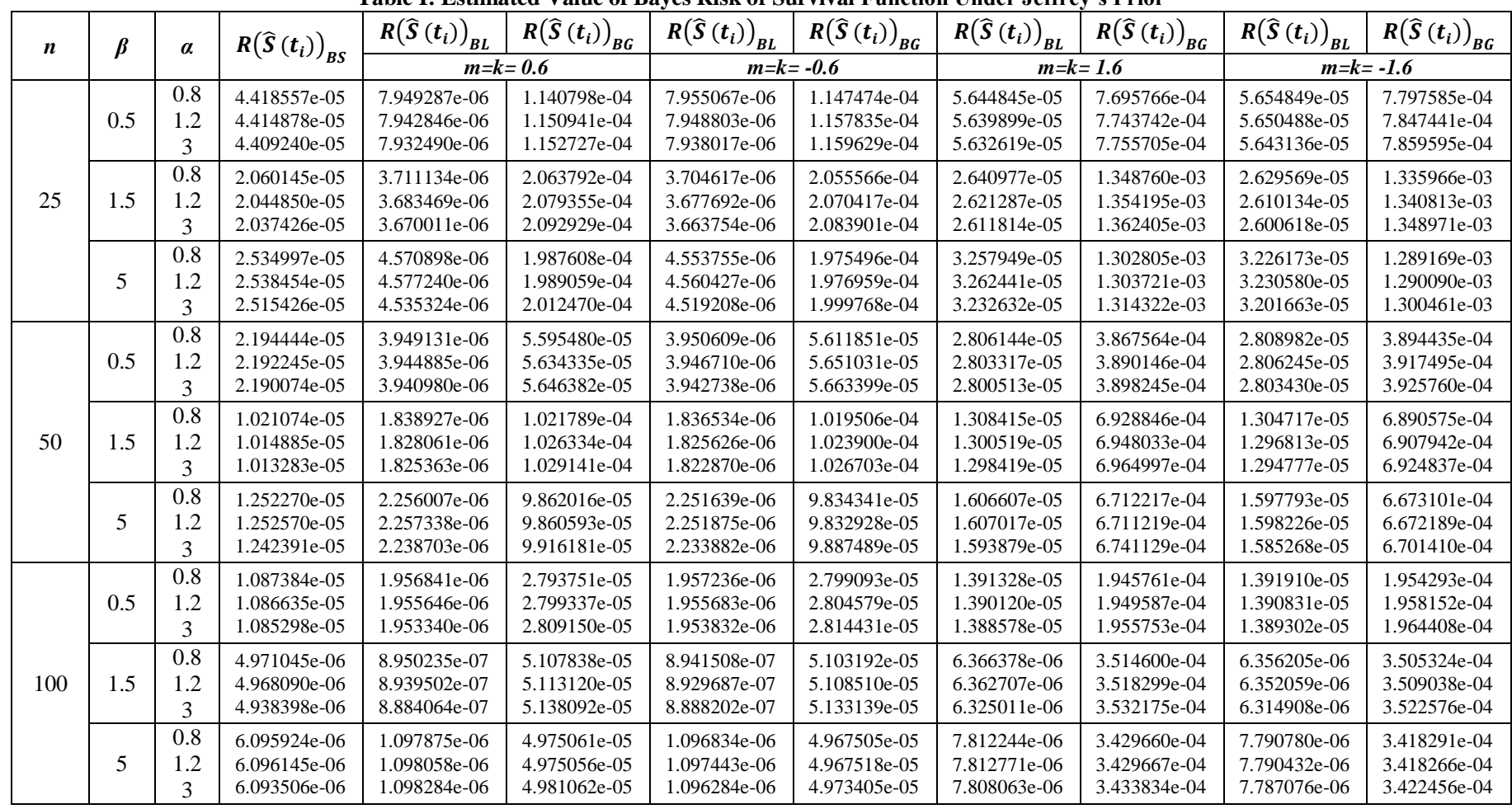

From table-I, the Bayes risk of survival function is minimum under the SELF, LLF, WLF and PLF when $\beta<1$ and $\alpha>1$ than $\beta<1$ and $\alpha<1$ and also minimum when $\beta>1$ and $\alpha>1$ than $\beta>1$ and $\alpha<1$ for $n=25$. The same behavior of the values of scale and shape parameters of the Bayes risk of survival function is reported when $n=50$ and $n=100$. The Bayes risk of survival function is maximum under the GELF, QLF and SLLF when $\beta<1$ and $\alpha>1$ than $\beta<1$ and $\alpha<1$ and also maximum when $\beta>1$ and $\alpha>1$ than $\beta>1$ and $\alpha<1$ for $n=25$. The same behavior of the values of scale and shape parameters of the Bayes risk of survival function is reported when $n=50$ and $n=100$. The Bayes risk of survival function for the Weibull distribution using noninformative prior (Jeffrey's) under LLF is better than other loss functions (SELF, GELF, PLF, QLF, WLF and SLLF) proposed in this study.

\begin{tabular}{|c|c|c|c|c|c|c|}
\hline \multicolumn{7}{|c|}{ Table-I (Continued) } \\
\hline$n$ & $\beta$ & $\alpha$ & $\boldsymbol{R}\left(\widehat{\boldsymbol{S}}\left(\boldsymbol{t}_{i}\right)\right)_{B Q}$ & $\boldsymbol{R}\left(\widehat{\boldsymbol{S}}\left(\boldsymbol{t}_{\boldsymbol{i}}\right)\right)_{B W}$ & $\boldsymbol{R}\left(\widehat{\boldsymbol{S}}\left(\boldsymbol{t}_{\boldsymbol{i}}\right)\right)_{B S L}$ & $R\left(\widehat{S}\left(t_{i}\right)\right)_{B P}$ \\
\hline 25 & 0.5 & $\begin{array}{l}0.8 \\
1.2 \\
\end{array}$ & $\begin{array}{l}5.430582 \mathrm{e}-04 \\
5.446594 \mathrm{e}-04 \\
\end{array}$ & $\begin{array}{l}1.002725 \mathrm{e}-04 \\
1.000657 \mathrm{e}-04\end{array}$ & $\begin{array}{l}6.431993 \mathrm{e}-04 \\
6.496146 \mathrm{e}-04 \\
\end{array}$ & $\begin{array}{l}9.998820 \mathrm{e}-05 \\
9.978865 \mathrm{e}-05 \\
\end{array}$ \\
\hline
\end{tabular}




\begin{tabular}{|c|c|c|c|c|c|c|}
\hline & & 3 & $5.454716 \mathrm{e}-04$ & $9.994241 \mathrm{e}-05$ & $6.506171 \mathrm{e}-04$ & $9.966426 \mathrm{e}-05$ \\
\hline & 1.5 & $\begin{array}{c}0.8 \\
1.2 \\
3\end{array}$ & $\begin{array}{l}9.018091 \mathrm{e}-04 \\
9.016163 \mathrm{e}-04 \\
9.060585 \mathrm{e}-04\end{array}$ & $\begin{array}{l}8.703055 \mathrm{e}-05 \\
8.647026 \mathrm{e}-05 \\
8.618781 \mathrm{e}-05\end{array}$ & $\begin{array}{l}1.174877 \mathrm{e}-03 \\
1.185809 \mathrm{e}-03 \\
1.193603 \mathrm{e}-03\end{array}$ & $\begin{array}{l}8.322924 \mathrm{e}-05 \\
8.268316 \mathrm{e}-05 \\
8.239871 \mathrm{e}-05\end{array}$ \\
\hline & 5 & $\begin{array}{c}0.8 \\
1.2 \\
3 \\
\end{array}$ & $\begin{array}{l}8.767137 \mathrm{e}-04 \\
8.772330 \mathrm{e}-04 \\
8.799160 \mathrm{e}-04\end{array}$ & $\begin{array}{l}1.046714 \mathrm{e}-04 \\
1.047858 \mathrm{e}-04 \\
1.038247 \mathrm{e}-04\end{array}$ & $\begin{array}{l}1.128818 \mathrm{e}-03 \\
1.129646 \mathrm{e}-03 \\
1.144923 \mathrm{e}-03\end{array}$ & $\begin{array}{l}9.965145 \mathrm{e}-05 \\
9.976102 \mathrm{e}-05 \\
9.886549 \mathrm{e}-05\end{array}$ \\
\hline \multirow{3}{*}{50} & 0.5 & $\begin{array}{c}0.8 \\
1.2 \\
3 \\
\end{array}$ & $\begin{array}{l}2.839965 \mathrm{e}-04 \\
2.851473 \mathrm{e}-04 \\
2.856845 \mathrm{e}-04\end{array}$ & $\begin{array}{l}5.024450 \mathrm{e}-05 \\
5.016683 \mathrm{e}-05 \\
5.011530 \mathrm{e}-05\end{array}$ & $\begin{array}{l}3.129930 \mathrm{e}-04 \\
3.152562 \mathrm{e}-04 \\
3.159438 \mathrm{e}-04\end{array}$ & $\begin{array}{l}5.013862 \mathrm{e}-05 \\
5.005998 \mathrm{e}-05 \\
5.000764 \mathrm{e}-05\end{array}$ \\
\hline & 1.5 & $\begin{array}{c}0.8 \\
1.2 \\
3 \\
\end{array}$ & $\begin{array}{l}4.897898 \mathrm{e}-04 \\
4.901040 \mathrm{e}-04 \\
4.910421 \mathrm{e}-04\end{array}$ & $\begin{array}{l}4.418979 \mathrm{e}-05 \\
4.398142 \mathrm{e}-05 \\
4.392888 \mathrm{e}-05\end{array}$ & $\begin{array}{l}5.735109 \mathrm{e}-04 \\
5.763870 \mathrm{e}-04 \\
5.780099 \mathrm{e}-04\end{array}$ & $\begin{array}{l}4.304629 \mathrm{e}-05 \\
4.283704 \mathrm{e}-05 \\
4.278338 \mathrm{e}-05\end{array}$ \\
\hline & 5 & $\begin{array}{c}0.8 \\
1.2 \\
3\end{array}$ & $\begin{array}{l}4.774553 \mathrm{e}-04 \\
4.773896 \mathrm{e}-04 \\
4.788424 \mathrm{e}-04\end{array}$ & $\begin{array}{l}5.282693 \mathrm{e}-05 \\
5.284461 \mathrm{e}-05 \\
5.245202 \mathrm{e}-05\end{array}$ & $\begin{array}{l}5.528001 \mathrm{e}-04 \\
5.527146 \mathrm{e}-04 \\
5.560573 \mathrm{e}-04\end{array}$ & $\begin{array}{l}5.136584 \mathrm{e}-05 \\
5.138345 \mathrm{e}-05 \\
5.100821 \mathrm{e}-05\end{array}$ \\
\hline \multirow{3}{*}{100} & 0.5 & $\begin{array}{c}0.8 \\
1.2 \\
3\end{array}$ & $\begin{array}{l}1.451893 \mathrm{e}-04 \\
1.454648 \mathrm{e}-04 \\
1.458374 \mathrm{e}-04\end{array}$ & $\begin{array}{l}2.468772 \mathrm{e}-05 \\
2.466553 \mathrm{e}-05 \\
2.463254 \mathrm{e}-05\end{array}$ & $\begin{array}{l}1.559766 \mathrm{e}-04 \\
1.562773 \mathrm{e}-04 \\
1.568464 \mathrm{e}-04\end{array}$ & $\begin{array}{l}2.465046 \mathrm{e}-05 \\
2.463037 \mathrm{e}-05 \\
2.459567 \mathrm{e}-05\end{array}$ \\
\hline & 1.5 & $\begin{array}{c}0.8 \\
1.2 \\
3 \\
\end{array}$ & $\begin{array}{l}2.562452 \mathrm{e}-04 \\
2.559646 \mathrm{e}-04 \\
2.568737 \mathrm{e}-04\end{array}$ & $\begin{array}{c}2.101833 \mathrm{e}-05 \\
2.132117 \mathrm{e}-052.122473 \mathrm{e}-05\end{array}$ & $\begin{array}{l}2.858378 \mathrm{e}-04 \\
2.861377 \mathrm{e}-04 \\
2.876036 \mathrm{e}-04\end{array}$ & $\begin{array}{l}2.100551 \mathrm{e}-05 \\
2.101833 \mathrm{e}-05 \\
2.090693 \mathrm{e}-05\end{array}$ \\
\hline & 5 & $\begin{array}{c}0.8 \\
1.2 \\
3\end{array}$ & $\begin{array}{l}2.503605 \mathrm{e}-04 \\
2.503600 \mathrm{e}-04 \\
2.506788 \mathrm{e}-04\end{array}$ & $\begin{array}{l}2.555391 \mathrm{e}-05 \\
2.555349 \mathrm{e}-05 \\
2.554422 \mathrm{e}-05\end{array}$ & $\begin{array}{l}3.374209 \mathrm{e}-04 \\
3.418266 \mathrm{e}-04 \\
3.422456 \mathrm{e}-04\end{array}$ & $\begin{array}{l}2.514899 \mathrm{e}-05 \\
2.514866 \mathrm{e}-05 \\
2.513914 \mathrm{e}-05\end{array}$ \\
\hline
\end{tabular}

The Bayes risk of survival function for the two parameter Weibull distribution using non-informative prior (Extension of Jeffrey's prior $\mathrm{p}=0.4$ ) under various loss functions is obtained and presented in Table-II.

Table-II: Estimated Value of Bayes Risk of Survival Function Under Extension of Jeffrey's Prior With p=0.4

\begin{tabular}{|c|c|c|c|c|c|c|c|c|c|c|c|}
\hline \multirow[t]{2}{*}{$n$} & \multirow[t]{2}{*}{$\beta$} & \multirow[t]{2}{*}{$\alpha$} & \multirow{2}{*}{$R\left(\widehat{S}\left(t_{i}\right)\right)_{B S}$} & $\boldsymbol{R}\left(\widehat{\boldsymbol{S}}\left(\boldsymbol{t}_{i}\right)\right)_{B L}$ & $R\left(\widehat{S}\left(t_{i}\right)\right)_{B G}$ & $R\left(\widehat{S}\left(t_{i}\right)\right)_{B L}$ & $\boldsymbol{R}\left(\widehat{\boldsymbol{S}}\left(\boldsymbol{t}_{i}\right)\right)_{B G}$ & $R\left(\widehat{S}\left(t_{i}\right)\right)_{B L}$ & $R\left(\widehat{S}\left(t_{i}\right)\right)_{B G}$ & $R\left(\widehat{S}\left(t_{i}\right)\right)_{B L}$ & $\boldsymbol{R}\left(\widehat{S}\left(\boldsymbol{t}_{i}\right)\right)_{B G}$ \\
\hline & & & & \multicolumn{2}{|c|}{$m=k=0.6$} & \multicolumn{2}{|c|}{$m=k=-0.6$} & \multicolumn{2}{|c|}{$m=k=1.6$} & \multicolumn{2}{|c|}{$m=k=-1.6$} \\
\hline \multirow{9}{*}{25} & & 0.8 & $4.418264 \mathrm{e}-05$ & $7.951277 \mathrm{e}-06$ & $1.143789 \mathrm{e}-04$ & $7.952369 \mathrm{e}-06$ & $1.145018 \mathrm{e}-04$ & $5.648547 \mathrm{e}-05$ & $7.730596 \mathrm{e}-04$ & $5.650439 \mathrm{e}-05$ & $7.766586 \mathrm{e}-04$ \\
\hline & 0.5 & 1.2 & $4.413772 \mathrm{e}-05$ & $7.941768 \mathrm{e}-06$ & $1.154511 \mathrm{e}-04$ & $7.945222 \mathrm{e}-06$ & $1.154639 \mathrm{e}-04$ & $5.640873 \mathrm{e}-05$ & $7.784868 \mathrm{e}-04$ & $5.646612 \mathrm{e}-05$ & $7.809085 \mathrm{e}-04$ \\
\hline & & 3 & $4.404854 \mathrm{e}-05$ & $7.923412 \mathrm{e}-06$ & $1.157142 \mathrm{e}-04$ & $7.931170 \mathrm{e}-06$ & $1.154994 \mathrm{e}-04$ & $5.625356 \mathrm{e}-05$ & $7.808143 \mathrm{e}-04$ & $5.639258 \mathrm{e}-05$ & $7.805746 \mathrm{e}-04$ \\
\hline & & 0.8 & $2.061202 \mathrm{e}-05$ & $3.712725 \mathrm{e}-06$ & $2.061290 \mathrm{e}-04$ & $3.707106 \mathrm{e}-06$ & $353 e-04$ & $2.641600 \mathrm{e}-05$ & $87 \mathrm{e}-03$ & $2.631630 \mathrm{e}-05$ & $1.338983 \mathrm{e}-03$ \\
\hline & 1.5 & 1.2 & $2.045094 \mathrm{e}-05$ & $3.683534 \mathrm{e}-06$ & $2.079124 \mathrm{e}-04$ & $3.678168 \mathrm{e}-06$ & $2.070656 \mathrm{e}-04$ & $2.620930 \mathrm{e}-05$ & $1.353787 \mathrm{e}-03$ & $2.611130 \mathrm{e}-05$ & $1.341220 \mathrm{e}-03$ \\
\hline & & 3 & $2.035465 \mathrm{e}-05$ & $3.666200 \mathrm{e}-06$ & $2.098520 \mathrm{e}-04$ & $3.660458 \mathrm{e}-06$ & $2.077133 \mathrm{e}-04$ & $2.608809 \mathrm{e}-05$ & $1.368405 \mathrm{e}-03$ & $2.598661 \mathrm{e}-05$ & $1.342412 \mathrm{e}-03$ \\
\hline & & 0.8 & $2.544246 \mathrm{e}-05$ & $4.585068 \mathrm{e}-06$ & $1.976968 \mathrm{e}-04$ & $4.573218 \mathrm{e}-06$ & $1.986604 \mathrm{e}-04$ & $3.264456 \mathrm{e}-05$ & $1.291498 \mathrm{e}-03$ & $3.243171 \mathrm{e}-05$ & $1.300715 \mathrm{e}-03$ \\
\hline & 5 & 1.2 & $2.546998 \mathrm{e}-05$ & $4.589944 \mathrm{e}-06$ & 1.9799 & $79 \mathrm{e}-06$ & 1.98 & $8 \mathrm{e}-05$ & $7 e-03$ & $2 \mathrm{e}-05$ & $1.300069 \mathrm{e}-03$ \\
\hline & & 3 & $2.522195 \mathrm{e}-05$ & $4.545363 \mathrm{e}-06$ & $2.006595 \mathrm{e}-04$ & $4.533379 \mathrm{e}-06$ & 2.006 & 3.2371 & $1.308150 \mathrm{e}-03$ & $3.214269 \mathrm{e}-05$ & $1.306909 \mathrm{e}-03$ \\
\hline \multirow{9}{*}{50} & & 0.8 & 2.1944 & $39 \mathrm{e}-06$ & 5.601 & $71 \mathrm{e}-06$ & $9 e-05$ & $2.806953 \mathrm{e}-05$ & $3.875531 \mathrm{e}-04$ & $2.808194 \mathrm{e}-05$ & $3.887384 \mathrm{e}-04$ \\
\hline & 0.5 & 1.2 & $2.191993 \mathrm{e}-05$ & $3.944592 \mathrm{e}-06$ & $2.801270 \mathrm{e}-05$ & $3.945811 \mathrm{e}-06$ & $5.645040 \mathrm{e}-05$ & $2.803355 \mathrm{e}-05$ & $3.899935 \mathrm{e}-04$ & $2.805498 \mathrm{e}-05$ & $3.908353 \mathrm{e}-04$ \\
\hline & & 3 & $2.188831 \mathrm{e}-05$ & $3.938181 \mathrm{e}-06$ & $5.655610 \mathrm{e}-05$ & $3.940725 \mathrm{e}-06$ & $5.653416 \mathrm{e}-05$ & $2.798321 \mathrm{e}-05$ & $3.911784 \mathrm{e}-04$ & $2.802438 \mathrm{e}-05$ & $3.911662 \mathrm{e}-04$ \\
\hline & & 0.8 & $1.021490 \mathrm{e}-05$ & $1.839696 \mathrm{e}-06$ & $1.021063 \mathrm{e}-04$ & $1.837510 \mathrm{e}-06$ & $1.020324 \mathrm{e}-04$ & $1.308679 \mathrm{e}-05$ & $6.918898 \mathrm{e}-04$ & $1.305477 \mathrm{e}-05$ & $6.900994 \mathrm{e}-04$ \\
\hline & 1.5 & 1.2 & $1.015071 \mathrm{e}-05$ & $1.828326 \mathrm{e}-06$ & $1.026109 \mathrm{e}-04$ & $1.826219 \mathrm{e}-06$ & $1.024135 \mathrm{e}-04$ & $1.300507 \mathrm{e}-05$ & $6.945226 \mathrm{e}-04$ & $1.297272 \mathrm{e}-05$ & $6.910896 \mathrm{e}-04$ \\
\hline & & 3 & $1.012834 \mathrm{e}-05$ & $1.824340 \mathrm{e}-06$ & $1.030257 \mathrm{e}-04$ & $1.822272 \mathrm{e}-06$ & $1.025282 \mathrm{e}-04$ & $1.297696 \mathrm{e}-05$ & $6.980871 \mathrm{e}-04$ & $1.294305 \mathrm{e}-05$ & $6.907315 \mathrm{e}-04$ \\
\hline & & 0.8 & $1.254886 \mathrm{e}-05$ & $2.259786 \mathrm{e}-06$ & $9.836419 \mathrm{e}-05$ & $2.257115 \mathrm{e}-06$ & $9.860942 \mathrm{e}-05$ & $1.608474 \mathrm{e}-05$ & $6.678004 \mathrm{e}-04$ & $1.602512 \mathrm{e}-05$ & $6.708030 \mathrm{e}-04$ \\
\hline & 5 & 1.2 & $1.254976 \mathrm{e}-05$ & $2.260657 \mathrm{e}-06$ & $9.838481 \mathrm{e}-05$ & $2.257093 \mathrm{e}-06$ & $9.856324 \mathrm{e}-05$ & $1.608768 \mathrm{e}-05$ & $6.681581 \mathrm{e}-04$ & $1.602590 \mathrm{e}-05$ & $6.702659 \mathrm{e}-04$ \\
\hline & & 3 & $1.244297 \mathrm{e}-05$ & $2.241480 \mathrm{e}-06$ & $9.901626 \mathrm{e}-05$ & $2.237819 \mathrm{e}-06$ & $9.903827 \mathrm{e}-05$ & $1.595187 \mathrm{e}-05$ & $6.721873 e-04$ & $1.588752 \mathrm{e}-05$ & $6.721568 \mathrm{e}-04$ \\
\hline \multirow{9}{*}{100} & & 0.8 & $1.087368 \mathrm{e}-05$ & $1.957148 \mathrm{e}-06$ & $2.795259 \mathrm{e}-05$ & $1.957043 \mathrm{e}-06$ & $2.797934 \mathrm{e}-05$ & $1.391498 \mathrm{e}-05$ & $1.947980 \mathrm{e}-04$ & $1.391742 \mathrm{e}-05$ & $1.952309 \mathrm{e}-04$ \\
\hline & 0.5 & 1.2 & $1.086559 \mathrm{e}-05$ & $1.955652 \mathrm{e}-06$ & $2.801270 \mathrm{e}-05$ & $1.955555 \mathrm{e}-06$ & $2.802917 \mathrm{e}-05$ & $1.390128 \mathrm{e}-05$ & $1.952413 \mathrm{e}-04$ & $1.390622 \mathrm{e}-05$ & $1.955478 \mathrm{e}-04$ \\
\hline & & 3 & $1.084968 \mathrm{e}-05$ & $1.952816 \mathrm{e}-06$ & $2.811993 \mathrm{e}-05$ & $1.953198 \mathrm{e}-06$ & $2.811482 \mathrm{e}-05$ & $1.388063 \mathrm{e}-05$ & $1.960143 \mathrm{e}-04$ & $1.388969 \mathrm{e}-05$ & $1.959930 \mathrm{e}-04$ \\
\hline & & 0.8 & $4.972178 \mathrm{e}-06$ & $8.951433 \mathrm{e}-07$ & $5.105587 \mathrm{e}-05$ & $8.945977 \mathrm{e}-07$ & $5.105608 \mathrm{e}-05$ & $6.367354 \mathrm{e}-06$ & $3.511366 \mathrm{e}-04$ & $6.358526 \mathrm{e}-06$ & $3.508723 \mathrm{e}-04$ \\
\hline & 1.5 & 1.2 & $4.968658 \mathrm{e}-06$ & $8.938724 \mathrm{e}-07$ & $5.112445 \mathrm{e}-05$ & $8.931692 \mathrm{e}-07$ & $5.109271 \mathrm{e}-05$ & $6.362863 \mathrm{e}-06$ & $3.517258 \mathrm{e}-04$ & $6.353202 \mathrm{e}-06$ & $3.510111 \mathrm{e}-04$ \\
\hline & & 3 & $4.937347 \mathrm{e}-06$ & $8.880986 \mathrm{e}-07$ & $5.141310 \mathrm{e}-05$ & $8.886763 \mathrm{e}-07$ & $5.129320 \mathrm{e}-05$ & $6.323291 \mathrm{e}-06$ & $3.536857 \mathrm{e}-04$ & $6.314049 \mathrm{e}-06$ & $3.517495 \mathrm{e}-04$ \\
\hline & & 0.8 & $6.102473 \mathrm{e}-06$ & $1.098843 \mathrm{e}-06$ & $4.967347 \mathrm{e}-05$ & $1.098290 \mathrm{e}-06$ & $4.975447 \mathrm{e}-05$ & $7.816730 \mathrm{e}-06$ & $3.418538 \mathrm{e}-04$ & $7.802339 \mathrm{e}-06$ & $3.429597 \mathrm{e}-04$ \\
\hline & 5 & 1.2 & $6.102067 \mathrm{e}-06$ & $1.098855 \mathrm{e}-06$ & $4.968270 \mathrm{e}-05$ & $1.098775 \mathrm{e}-06$ & $4.974532 \mathrm{e}-05$ & $7.816968 \mathrm{e}-06$ & $3.420005 \mathrm{e}-04$ & $7.801363 \mathrm{e}-06$ & $3.428157 \mathrm{e}-04$ \\
\hline & & 3 & $6.098443 \mathrm{e}-06$ & $1.098966 \mathrm{e}-06$ & $4.976913 \mathrm{e}-05$ & $1.097453 \mathrm{e}-06$ & $4.977976 \mathrm{e}-05$ & $7.811144 \mathrm{e}-06$ & $3.427795 \mathrm{e}-04$ & $7.795996 e-06$ & $3.428744 \mathrm{e}-04$ \\
\hline
\end{tabular}

From table-II, the Bayes risk of survival function is minimum under the SELF, LLF, WLF and PLF when $\beta<1$ and $\alpha>1$ than $\beta<1$ and $\alpha<1$ and also minimum when $\beta>1$ and $\alpha>1$ than $\beta>1$ and $\alpha<1$ for $n=25$. The same behavior of the values of scale and shape parameters of the Bayes risk of survival function is reported when $n=50$ and $n=100$. The Bayes risk of survival function 
is maximum under the GELF, QLF and SLLF when $\beta<1$ and $\alpha>1$ than $\beta<1$ and $\alpha<1$ and also maximum when $\beta>1$ and $\alpha>1$ than $\beta>1$ and $\alpha<1$ for $n=25$. The same behavior of the values of scale and shape parameters of the Bayes risk of survival function is reported when $n=50$ and $n=100$. The Bayes risk of survival function for the Weibull distribution using non-informative prior (Extension of Jeffrey's prior $\mathrm{p}=0.4$ ) under LLF is better than other loss functions (SELF, GELF, PLF, QLF, WLF and SLLF) proposed in this study.

\begin{tabular}{|c|c|c|c|c|c|c|}
\hline \multicolumn{7}{|c|}{ Table-II (Continued) } \\
\hline$n$ & $\beta$ & $\alpha$ & $R\left(\widehat{S}\left(t_{i}\right)\right)_{B Q}$ & $R\left(\widehat{S}\left(t_{i}\right)\right)_{B W}$ & $R\left(\widehat{S}\left(t_{i}\right)\right)_{B S L}$ & $R\left(\widehat{S}\left(t_{i}\right)\right)_{B P}$ \\
\hline \multirow{9}{*}{25} & \multirow{3}{*}{0.5} & 0.8 & $5.459610 \mathrm{e}-04$ & $1.004911 \mathrm{e}-04$ & $6.433418 \mathrm{e}-04$ & $9.978789 \mathrm{e}-05$ \\
\hline & & 1.2 & $5.479817 \mathrm{e}-04$ & $1.002235 \mathrm{e}-04$ & $6.497061 \mathrm{e}-04$ & $9.960089 \mathrm{e}-05$ \\
\hline & & 3 & $5.496685 \mathrm{e}-04$ & $9.993274 \mathrm{e}-05$ & $6.505372 \mathrm{e}-04$ & $9.948781 \mathrm{e}-05$ \\
\hline & \multirow{3}{*}{1.5} & 0.8 & $8.994598 \mathrm{e}-04$ & $8.695274 \mathrm{e}-05$ & $1.174953 \mathrm{e}-03$ & $8.337316 \mathrm{e}-05$ \\
\hline & & 1.2 & $9.012026 \mathrm{e}-04$ & $8.643445 \mathrm{e}-05$ & $1.185813 \mathrm{e}-03$ & $8.273105 \mathrm{e}-05$ \\
\hline & & 3 & $9.106624 \mathrm{e}-04$ & $8.625505 \mathrm{e}-05$ & $1.193217 \mathrm{e}-03$ & $8.219517 \mathrm{e}-05$ \\
\hline & \multirow{3}{*}{5} & 0.8 & $8.680712 \mathrm{e}-04$ & $1.042696 \mathrm{e}-04$ & $1.128951 \mathrm{e}-03$ & $1.004257 \mathrm{e}-04$ \\
\hline & & 1.2 & $8.698079 \mathrm{e}-04$ & $1.044325 \mathrm{e}-04$ & $1.129829 \mathrm{e}-03$ & $1.004520 \mathrm{e}-04$ \\
\hline & & 3 & $8.752621 \mathrm{e}-04$ & $1.035854 \mathrm{e}-04$ & $1.145146 \mathrm{e}-03$ & $9.935577 \mathrm{e}-05$ \\
\hline \multirow{9}{*}{50} & \multirow{3}{*}{0.5} & 0.8 & $2.848060 \mathrm{e}-04$ & $5.029510 \mathrm{e}-05$ & $3.130333 \mathrm{e}-04$ & $5.009032 \mathrm{e}-05$ \\
\hline & & 1.2 & $2.861169 \mathrm{e}-04$ & 5.020191e-05 & $1.562863 \mathrm{e}-04$ & $5.001266 \mathrm{e}-05$ \\
\hline & & 3 & $2.870143 \mathrm{e}-04$ & $5.010758 \mathrm{e}-05$ & $3.159232 \mathrm{e}-04$ & $4.996011 \mathrm{e}-05$ \\
\hline & \multirow{3}{*}{1.5} & 0.8 & $4.888604 \mathrm{e}-04$ & $4.416229 \mathrm{e}-05$ & $7.105373 \mathrm{e}-04$ & $4.310061 \mathrm{e}-05$ \\
\hline & & 1.2 & $4.898454 \mathrm{e}-04$ & $4.396701 \mathrm{e}-05$ & $5.763926 \mathrm{e}-04$ & $4.286149 \mathrm{e}-05$ \\
\hline & & 3 & $4.925372 \mathrm{e}-04$ & $4.394636 \mathrm{e}-05$ & $5.779224 \mathrm{e}-04$ & $4.272835 \mathrm{e}-05$ \\
\hline & \multirow{3}{*}{5} & 0.8 & $4.742839 \mathrm{e}-04$ & $5.270112 \mathrm{e}-05$ & $5.528242 \mathrm{e}-04$ & $5.161245 \mathrm{e}-05$ \\
\hline & & 1.2 & $4.746465 \mathrm{e}-04$ & $5.273378 \mathrm{e}-05$ & $5.527550 \mathrm{e}-04$ & $5.160582 \mathrm{e}-05$ \\
\hline & & 3 & $4.771079 \mathrm{e}-04$ & $5.237518 \mathrm{e}-05$ & $5.561083 \mathrm{e}-04$ & $5.116975 \mathrm{e}-05$ \\
\hline \multirow{9}{*}{100} & \multirow{3}{*}{0.5} & 0.8 & $1.454291 \mathrm{e}-04$ & $2.469975 \mathrm{e}-05$ & $1.559865 \mathrm{e}-04$ & $2.463759 \mathrm{e}-05$ \\
\hline & & 1.2 & $1.457675 \mathrm{e}-04$ & $2.467443 \mathrm{e}-05$ & $1.562863 \mathrm{e}-04$ & $2.461759 \mathrm{e}-05$ \\
\hline & & 3 & $1.463068 \mathrm{e}-04$ & $2.463167 \mathrm{e}-05$ & $1.568424 \mathrm{e}-04$ & $2.458112 \mathrm{e}-05$ \\
\hline & \multirow{3}{*}{1.5} & 0.8 & $2.556297 \mathrm{e}-04$ & $2.132688 \mathrm{e}-05$ & $2.858452 \mathrm{e}-04$ & $2.103411 \mathrm{e}-05$ \\
\hline & & 1.2 & $2.561402 \mathrm{e}-04$ & $2.131703 e-05$ & $2.861406 \mathrm{e}-04$ & $2.101306 \mathrm{e}-05$ \\
\hline & & 3 & $2.573529 \mathrm{e}-04$ & $2.122902 \mathrm{e}-05$ & $2.875835 \mathrm{e}-04$ & $2.089274 \mathrm{e}-05$ \\
\hline & \multirow{3}{*}{5} & 0.8 & $2.492257 \mathrm{e}-04$ & $2.552004 \mathrm{e}-05$ & $2.781617 \mathrm{e}-04$ & $2.521779 \mathrm{e}-05$ \\
\hline & & 1.2 & $2.493717 \mathrm{e}-04$ & $2.552407 \mathrm{e}-05$ & $2.781684 \mathrm{e}-04$ & $2.521088 \mathrm{e}-05$ \\
\hline & & 3 & $2.500606 \mathrm{e}-04$ & $2.552329 \mathrm{e}-05$ & $2.785034 \mathrm{e}-04$ & $2.518405 \mathrm{e}-05$ \\
\hline
\end{tabular}

The Bayes risk of survival function for the two parameter Weibull distribution using non-informative prior (Extension of Jeffrey's prior $\mathrm{p}=1.4$ ) under various loss functions is obtained and presented in Table-III.

Table-III: Estimated Value of Bayes Risk of Survival Function Under Extension of Jeffrey's Prior With p=1.4

\begin{tabular}{|c|c|c|c|c|c|c|c|c|c|c|c|}
\hline \multirow{2}{*}{$n$} & \multirow{2}{*}{$\beta$} & \multirow{2}{*}{$\alpha$} & \multirow{2}{*}{$\boldsymbol{R}\left(\widehat{S}\left(t_{i}\right)\right)_{B S}$} & $R\left(\widehat{S}\left(t_{i}\right)\right)_{B L}$ & $\boldsymbol{R}\left(\widehat{\boldsymbol{S}}\left(\boldsymbol{t}_{i}\right)\right)_{B G}$ & $R\left(\widehat{S}\left(t_{i}\right)\right)_{B L}$ & $R\left(\widehat{S}\left(t_{i}\right)\right)_{B G}$ & $R\left(\widehat{S}\left(t_{i}\right)\right)_{B L}$ & $R\left(\widehat{S}\left(t_{i}\right)\right)_{B G}$ & $\boldsymbol{R}\left(\widehat{\boldsymbol{S}}\left(\boldsymbol{t}_{i}\right)\right)_{B L}$ & $\boldsymbol{R}\left(\widehat{\boldsymbol{S}}\left(\boldsymbol{t}_{i}\right)\right)_{B G}$ \\
\hline & & & & \multicolumn{2}{|c|}{$m=k=0.6$} & \multicolumn{2}{|c|}{$m=k=-0.6$} & \multicolumn{2}{|c|}{$m=k=1.6$} & \multicolumn{2}{|c|}{$m=k=-1.6$} \\
\hline \multirow{9}{*}{25} & & 0.8 & 4.019 & $7.195666 \mathrm{e}-06$ & $9.254668 \mathrm{e}-05$ & $7.274419 \mathrm{e}-06$ & $1.003502 \mathrm{e}-04$ & $5.071197 \mathrm{e}-05$ & $6.265133 \mathrm{e}-04$ & $5.220453 \mathrm{e}-05$ & $7.631473 \mathrm{e}-04$ \\
\hline & 0.5 & 1.2 & $4.081042 \mathrm{e}-05$ & $7.309812 \mathrm{e}-06$ & $9.156056 \mathrm{e}-05$ & $7.381765 \mathrm{e}-06$ & $9.960794 \mathrm{e}-05$ & $5.155797 \mathrm{e}-05$ & $6.205571 \mathrm{e}-04$ & $5.292127 \mathrm{e}-05$ & $7.722313 \mathrm{e}-04$ \\
\hline & & 3 & $4.124825 \mathrm{e}-05$ & $7.391420 \mathrm{e}-06$ & $9.139340 \mathrm{e}-05$ & $7.457997 \mathrm{e}-06$ & $9.952742 \mathrm{e}-05$ & $5.216591 \mathrm{e}-05$ & $6.190843 e-04$ & $5.342138 \mathrm{e}-05$ & $7.774705 \mathrm{e}-04$ \\
\hline & & 0.8 & $2.048468 \mathrm{e}-05$ & $3.692286 \mathrm{e}-06$ & $2.080346 \mathrm{e}-04$ & $3.681450 \mathrm{e}-06$ & $2.030701 \mathrm{e}-04$ & $2.630710 \mathrm{e}-05$ & $38 \mathrm{e}-03$ & $2.610174 \mathrm{e}-05$ & $1.311294 \mathrm{e}-03$ \\
\hline & 1.5 & 1.2 & $2.040530 \mathrm{e}-05$ & $3.677989 \mathrm{e}-06$ & $2.080015 \mathrm{e}-04$ & $3.667303 \mathrm{e}-06$ & $2.069221 \mathrm{e}-04$ & $2.620071 \mathrm{e}-05$ & $1.355560 \mathrm{e}-03$ & $2.600505 \mathrm{e}-05$ & $1.339200 \mathrm{e}-03$ \\
\hline & & 3 & $2.037141 \mathrm{e}-05$ & $3.671594 \mathrm{e}-06$ & $2.078796 \mathrm{e}-04$ & $3.661380 \mathrm{e}-06$ & $2.097931 \mathrm{e}-04$ & $2.615425 \mathrm{e}-05$ & $1.348037 \mathrm{e}-03$ & $2.596479 \mathrm{e}-05$ & $1.363586 \mathrm{e}-03$ \\
\hline & & 0.8 & $2.483246 \mathrm{e}-05$ & $4.485718 \mathrm{e}-06$ & $2.011125 \mathrm{e}-04$ & $4.453528 \mathrm{e}-06$ & $1.930701 \mathrm{e}-04$ & $3.206701 \mathrm{e}-05$ & $1.334665 \mathrm{e}-03$ & $3.146427 \mathrm{e}-05$ & $1.249242 \mathrm{e}-03$ \\
\hline & 5 & 1.2 & $2.501656 \mathrm{e}-05$ & $4.516753 \mathrm{e}-06$ & $2.004579 \mathrm{e}-04$ & $4.488492 \mathrm{e}-06$ & $1.950698 \mathrm{e}-04$ & $3.227242 \mathrm{e}-05$ & $1.323134 \mathrm{e}-03$ & $3.172733 \mathrm{e}-05$ & $1.266420 \mathrm{e}-03$ \\
\hline & & 3 & $2.488726 \mathrm{e}-05$ & $4.492594 \mathrm{e}-06$ & $2.021528 \mathrm{e}-04$ & $4.466160 \mathrm{e}-06$ & $1.984954 \mathrm{e}-04$ & $3.207803 \mathrm{e}-05$ & $1.324674 \mathrm{e}-03$ & $3.158856 \mathrm{e}-05$ & $1.287721 \mathrm{e}-03$ \\
\hline \multirow{9}{*}{50} & & 0.8 & $2.145693 \mathrm{e}-05$ & $3.853244 \mathrm{e}-06$ & $5.279069 \mathrm{e}-05$ & $3.871080 \mathrm{e}-06$ & $5.505754 \mathrm{e}-05$ & 2.728 & $3 e-04$ & $2.762689 \mathrm{e}-05$ & $54 \mathrm{e}-04$ \\
\hline & 0.5 & 1.2 & $2.158468 \mathrm{e}-05$ & $3.877482 \mathrm{e}-06$ & $5.292139 \mathrm{e}-05$ & $3.892687 \mathrm{e}-06$ & $5.547203 \mathrm{e}-05$ & $2.747568 \mathrm{e}-05$ & $3.591671 \mathrm{e}-04$ & $2.776170 \mathrm{e}-05$ & $3.982879 \mathrm{e}-04$ \\
\hline & & 3 & $2.165222 \mathrm{e}-05$ & $3.890605 \mathrm{e}-06$ & $5.291033 \mathrm{e}-05$ & $3.903521 \mathrm{e}-06$ & $5.559756 \mathrm{e}-05$ & $2.758394 \mathrm{e}-05$ & $3.587828 \mathrm{e}-04$ & $2.782471 \mathrm{e}-05$ & $4.001062 \mathrm{e}-04$ \\
\hline & & 0.8 & $1.016711 \mathrm{e}-05$ & $1.831780 \mathrm{e}-06$ & $1.026142 \mathrm{e}-04$ & $1.827907 \mathrm{e}-06$ & $1.012338 \mathrm{e}-04$ & $1.304347 \mathrm{e}-05$ & $6.998261 \mathrm{e}-04$ & $1.297625 \mathrm{e}-05$ & $6.806801 \mathrm{e}-04$ \\
\hline & 1.5 & 1.2 & $1.012906 \mathrm{e}-05$ & $1.825454 \mathrm{e}-06$ & $1.027138 \mathrm{e}-04$ & $1.821627 \mathrm{e}-06$ & $1.022670 \mathrm{e}-04$ & $1.299208 \mathrm{e}-05$ & $6.959424 \mathrm{e}-04$ & $1.293038 \mathrm{e}-05$ & $6.894344 \mathrm{e}-04$ \\
\hline & & 3 & $1.012676 \mathrm{e}-05$ & $1.824822 \mathrm{e}-06$ & $1.026475 \mathrm{e}-04$ & $1.821435 \mathrm{e}-06$ & $1.029266 \mathrm{e}-04$ & $1.298649 \mathrm{e}-05$ & $6.929179 \mathrm{e}-04$ & $1.293009 \mathrm{e}-05$ & $6.960509 \mathrm{e}-04$ \\
\hline & & 0.8 & $1.238927 \mathrm{e}-05$ & $2.233799 \mathrm{e}-06$ & $9.911295 \mathrm{e}-05$ & $2.225638 \mathrm{e}-06$ & $9.727624 \mathrm{e}-05$ & $1.593319 \mathrm{e}-05$ & $6.802886 \mathrm{e}-04$ & $1.577052 \mathrm{e}-05$ & $6.555262 \mathrm{e}-04$ \\
\hline & 5 & 1.2 & $1.243190 \mathrm{e}-05$ & $2.241706 \mathrm{e}-06$ & $9.893832 \mathrm{e}-05$ & $2.233691 \mathrm{e}-06$ & $9.769929 \mathrm{e}-05$ & $1.597987 \mathrm{e}-05$ & $6.767546 \mathrm{e}-04$ & $1.583372 \mathrm{e}-05$ & $6.601491 \mathrm{e}-04$ \\
\hline & & 3 & $1.235701 \mathrm{e}-05$ & $2.227848 \mathrm{e}-06$ & $9.935501 \mathrm{e}-05$ & $2.220625 \mathrm{e}-06$ & $9.852013 \mathrm{e}-05$ & $1.587565 \mathrm{e}-05$ & $6.771665 \mathrm{e}-04$ & $1.574501 \mathrm{e}-05$ & $6.662905 \mathrm{e}-04$ \\
\hline
\end{tabular}




\begin{tabular}{|c|c|c|c|c|c|c|c|c|c|c|c|}
\hline \multirow{3}{*}{100} & 0.5 & $\begin{array}{c}0.8 \\
1.2 \\
3\end{array}$ & $\begin{array}{l}1.077262 \mathrm{e}-05 \\
1.080121 \mathrm{e}-05 \\
1.080757 \mathrm{e}-05\end{array}$ & $\begin{array}{l}1.936826 \mathrm{e}-06 \\
1.942580 \mathrm{e}-06 \\
1.944256 \mathrm{e}-06\end{array}$ & $\begin{array}{l}2.720014 \mathrm{e}-05 \\
2.719992 \mathrm{e}-05 \\
2.723254 \mathrm{e}-05\end{array}$ & $\begin{array}{l}1.941105 \mathrm{e}-06 \\
1.945391 \mathrm{e}-06 \\
1.946902 \mathrm{e}-06\end{array}$ & $\begin{array}{l}2.783694 \mathrm{e}-05 \\
2.790893 \mathrm{e}-05 \\
2.800863 \mathrm{e}-05\end{array}$ & $\begin{array}{l}1.374935 \mathrm{e}-05 \\
1.379012 \mathrm{e}-05 \\
1.380591 \mathrm{e}-05\end{array}$ & $\begin{array}{l}1.874392 \mathrm{e}-04 \\
1.872126 \mathrm{e}-04 \\
1.872015 \mathrm{e}-04\end{array}$ & $\begin{array}{l}1.382584 \mathrm{e}-05 \\
1.385280 \mathrm{e}-05 \\
1.385690 \mathrm{e}-05\end{array}$ & $\begin{array}{l}1.973618 \mathrm{e}-04 \\
1.982822 \mathrm{e}-04 \\
1.992950 \mathrm{e}-04\end{array}$ \\
\hline & 1.5 & $\begin{array}{c}0.8 \\
1.2 \\
3\end{array}$ & $\begin{array}{l}4.959605 \mathrm{e}-06 \\
4.963100 \mathrm{e}-06 \\
4.936933 \mathrm{e}-06\end{array}$ & $\begin{array}{l}8.930254 \mathrm{e}-07 \\
8.931556 \mathrm{e}-07 \\
8.882351 \mathrm{e}-07\end{array}$ & $\begin{array}{l}5.121740 \mathrm{e}-05 \\
5.116042 \mathrm{e}-05 \\
5.130655 \mathrm{e}-05\end{array}$ & $\begin{array}{l}8.919462 \mathrm{e}-07 \\
8.921013 \mathrm{e}-07 \\
8.884567 \mathrm{e}-07\end{array}$ & $\begin{array}{l}5.081238 \mathrm{e}-05 \\
5.104559 \mathrm{e}-05 \\
5.140322 \mathrm{e}-05\end{array}$ & $\begin{array}{l}6.355990 \mathrm{e}-06 \\
6.359545 \mathrm{e}-06 \\
6.325311 \mathrm{e}-06\end{array}$ & $\begin{array}{l}3.537782 \mathrm{e}-04 \\
3.522811 \mathrm{e}-04 \\
3.521742 \mathrm{e}-04\end{array}$ & $\begin{array}{l}6.338019 \mathrm{e}-06 \\
6.342392 \mathrm{e}-06 \\
6.310529 \mathrm{e}-06\end{array}$ & $\begin{array}{l}3.477657 \mathrm{e}-04 \\
3.503815 \mathrm{e}-04 \\
3.533005 \mathrm{e}-04\end{array}$ \\
\hline & 5 & $\begin{array}{c}0.8 \\
1.2 \\
3\end{array}$ & $\begin{array}{l}6.062397 \mathrm{e}-06 \\
6.072538 \mathrm{e}-06 \\
6.076675 \mathrm{e}-06\end{array}$ & $\begin{array}{l}1.092020 \mathrm{e}-06 \\
1.094172 \mathrm{e}-06 \\
1.095228 \mathrm{e}-06\end{array}$ & $\begin{array}{l}4.990905 \mathrm{e}-05 \\
4.985733 \mathrm{e}-05 \\
4.986881 \mathrm{e}-05\end{array}$ & $\begin{array}{l}1.090380 \mathrm{e}-06 \\
1.092852 \mathrm{e}-06 \\
1.093182 \mathrm{e}-06\end{array}$ & $\begin{array}{l}4.935539 \mathrm{e}-05 \\
4.948509 \mathrm{e}-05 \\
4.963245 \mathrm{e}-05\end{array}$ & $\begin{array}{l}7.779106 \mathrm{e}-06 \\
7.789939 \mathrm{e}-06 \\
7.792230 \mathrm{e}-06\end{array}$ & $\begin{array}{l}3.459455 \mathrm{e}-04 \\
3.448452 \mathrm{e}-04 \\
3.443835 \mathrm{e}-04\end{array}$ & $\begin{array}{l}7.738283 \mathrm{e}-06 \\
7.752663 \mathrm{e}-06 \\
7.759888 \mathrm{e}-06\end{array}$ & $\begin{array}{l}3.379859 \mathrm{e}-04 \\
3.394944 \mathrm{e}-04 \\
3.410023 \mathrm{e}-04\end{array}$ \\
\hline
\end{tabular}

From table-III, the Bayes risk of survival function is minimum under the SELF, LLF, WLF and PLF when $\beta<1$ and $\alpha<1$ than $\beta<1$ and $\alpha>1$ and also minimum when $\beta>1$ and $\alpha<1$ than $\beta>1$ and $\alpha>1$ for $n=25$. The same behavior of the values of scale and shape parameters of the Bayes risk of survival function is reported when $n=50$ and $n=100$. The Bayes risk of survival function is maximum under the GELF, QLF and SLLF when $\beta<1$ and $\alpha>1$ than $\beta<1$ and $\alpha<1$ and also maximum when $\beta>1$ and $\alpha>1$ than $\beta>1$ and $\alpha<1$ for $n=25$. The same behavior of the values of scale and shape parameters of the Bayes risk of survival function is reported when $n=50$ and $n=100$. The Bayes risk of survival function for the Weibull distribution using non-informative prior (Extension of Jeffrey's prior $\mathrm{p}=1.4$ ) under LLF is better than other loss functions (SELF, GELF, PLF, QLF, WLF and SLLF) proposed in this study.

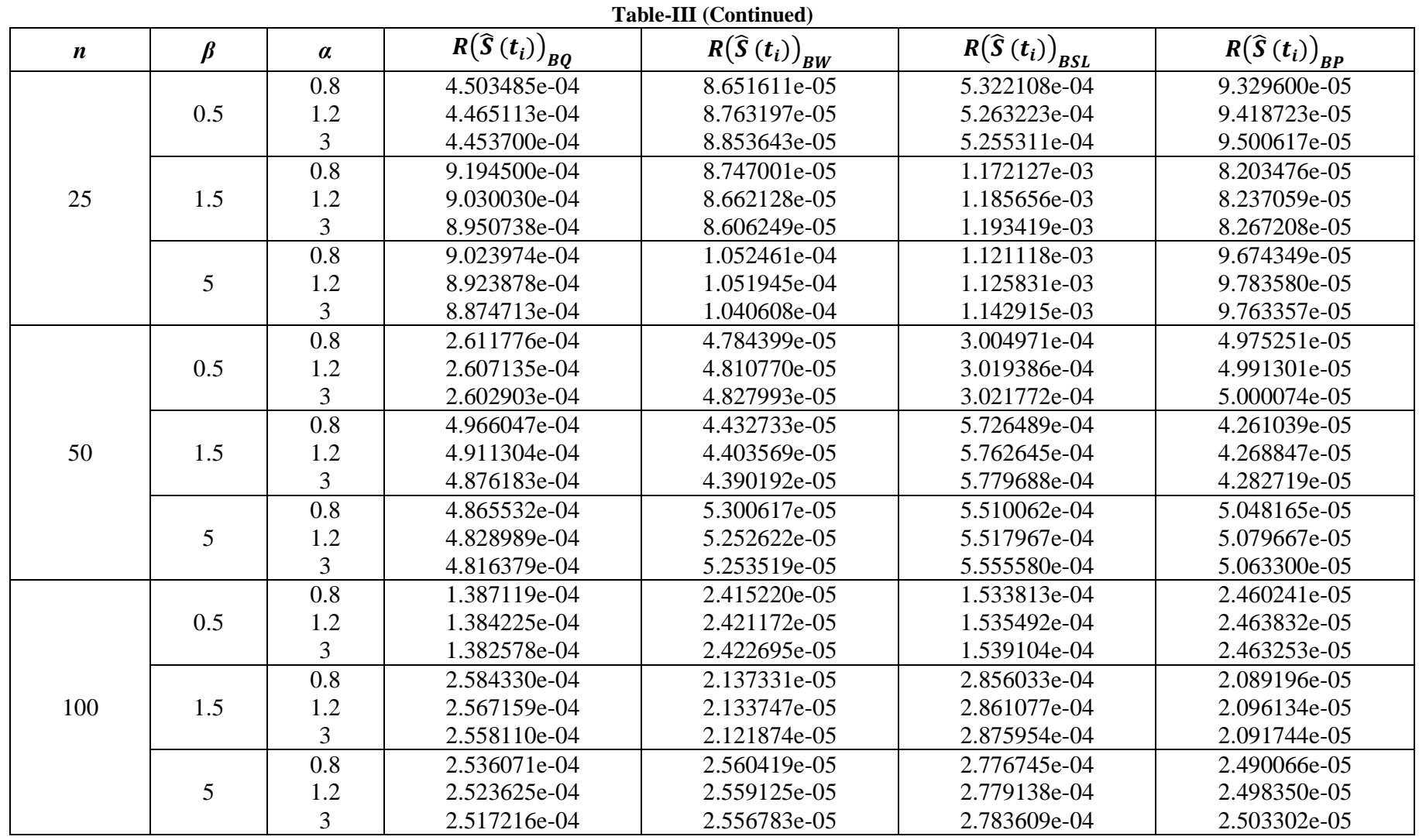

The Bayes risk of survival function for the two parameter Weibull distribution using informative prior (Lognormal-Inverted Gamma prior) under various loss functions is obtained and presented in Table-IV.

Table-IV: Estimated Value of Bayes Risk of Survival Function Under Lognormal-Inverted Gamma Prior With Hyperparameters a =0.4, $b=0.6$ and $\mathbf{c}=\mathbf{0 . 9}$.

\begin{tabular}{|c|c|c|c|c|c|c|c|c|c|c|c|}
\hline \multirow[t]{2}{*}{$n$} & \multirow[t]{2}{*}{$\beta$} & \multirow[t]{2}{*}{$\alpha$} & \multirow{2}{*}{$R\left(\widehat{S}\left(t_{i}\right)\right)_{B S}$} & $R\left(\widehat{S}\left(t_{i}\right)\right)_{B L}$ & $R\left(\widehat{S}\left(t_{i}\right)\right)_{B G}$ & $R\left(\widehat{S}\left(t_{i}\right)\right)_{B L}$ & $R\left(\widehat{S}\left(t_{i}\right)\right)_{B G}$ & $R\left(\widehat{S}\left(t_{i}\right)\right)_{B L}$ & $R\left(\widehat{S}\left(t_{i}\right)\right)_{B G}$ & $R\left(\widehat{S}\left(t_{i}\right)\right)_{B L}$ & \multirow{2}{*}{$\begin{array}{l}\boldsymbol{R}\left(\widehat{S}\left(t_{i}\right)\right)_{B G} \\
-1.6\end{array}$} \\
\hline & & & & \multicolumn{2}{|c|}{$m=k=0.6$} & \multicolumn{2}{|c|}{$m=k=-0.6$} & \multicolumn{2}{|c|}{$m=k=1.6$} & $m=k=-1.6$ & \\
\hline 25 & 0.5 & $\begin{array}{c}0.8 \\
1.2 \\
3\end{array}$ & $\begin{array}{l}4.392844 \mathrm{e}-05 \\
4.394993 \mathrm{e}-05 \\
4.373916 \mathrm{e}-05\end{array}$ & $\begin{array}{l}7.917331 \mathrm{e}-06 \\
7.916009 \mathrm{e}-06 \\
7.866446 \mathrm{e}-06\end{array}$ & $\begin{array}{l}1.152535 \mathrm{e}-04 \\
1.165691 \mathrm{e}-04 \\
1.168095 \mathrm{e}-04\end{array}$ & $\begin{array}{l}7.894742 \mathrm{e}-06 \\
7.903719 \mathrm{e}-06 \\
7.876993 \mathrm{e}-06\end{array}$ & $\begin{array}{l}1.123090 \mathrm{e}-04 \\
1.128129 \mathrm{e}-04 \\
1.119028 \mathrm{e}-04\end{array}$ & $\begin{array}{l}5.639152 \mathrm{e}-05 \\
5.631801 \mathrm{e}-05 \\
5.582904 \mathrm{e}-05\end{array}$ & $\begin{array}{l}7.894993 \mathrm{e}-04 \\
7.985373 \mathrm{e}-04 \\
8.046728 \mathrm{e}-04\end{array}$ & $\begin{array}{l}5.596060 \mathrm{e}-05 \\
5.608506 \mathrm{e}-05 \\
5.602908 \mathrm{e}-05\end{array}$ & $\begin{array}{l}7.557525 \mathrm{e}-04 \\
7.561645 \mathrm{e}-04 \\
7.480643 \mathrm{e}-04\end{array}$ \\
\hline
\end{tabular}




\begin{tabular}{|c|c|c|c|c|c|c|c|c|c|c|c|}
\hline & 1.5 & $\begin{array}{c}0.8 \\
1.2 \\
3\end{array}$ & $\begin{array}{l}2.061261 \mathrm{e}-05 \\
2.040927 \mathrm{e}-05 \\
2.018367 \mathrm{e}-05\end{array}$ & $\begin{array}{l}3.713256 \mathrm{e}-06 \\
3.676872 \mathrm{e}-06 \\
3.636344 \mathrm{e}-06\end{array}$ & $\begin{array}{l}2.060233 \mathrm{e}-04 \\
2.089804 \mathrm{e}-04 \\
2.126291 \mathrm{e}-04\end{array}$ & $\begin{array}{l}3.706871 \mathrm{e}-06 \\
3.669893 \mathrm{e}-06 \\
3.629005 \mathrm{e}-06\end{array}$ & $\begin{array}{l}2.059430 \mathrm{e}-04 \\
2.057394 \mathrm{e}-04 \\
2.025831 \mathrm{e}-04\end{array}$ & $\begin{array}{l}2.642838 \mathrm{e}-05 \\
2.617052 \mathrm{e}-05 \\
2.588875 \mathrm{e}-05\end{array}$ & $\begin{array}{l}1.344131 \mathrm{e}-03 \\
1.364980 \mathrm{e}-03 \\
1.404728 \mathrm{e}-03\end{array}$ & $\begin{array}{l}2.630542 \mathrm{e}-05 \\
2.604458 \mathrm{e}-05 \\
2.575019 \mathrm{e}-05\end{array}$ & $\begin{array}{l}1.340828 \mathrm{e}-03 \\
1.329003 \mathrm{e}-03 \\
1.297368 \mathrm{e}-03\end{array}$ \\
\hline & 5 & $\begin{array}{c}0.8 \\
1.2 \\
3\end{array}$ & $\begin{array}{l}2.544257 \mathrm{e}-05 \\
2.542853 \mathrm{e}-05 \\
2.506356 \mathrm{e}-05\end{array}$ & $\begin{array}{l}4.585117 \mathrm{e}-06 \\
4.583613 \mathrm{e}-06 \\
4.520212 \mathrm{e}-06\end{array}$ & $\begin{array}{l}1.975747 \mathrm{e}-04 \\
1.986550 \mathrm{e}-04 \\
2.028159 \mathrm{e}-04\end{array}$ & $\begin{array}{l}4.572884 \mathrm{e}-06 \\
4.569443 \mathrm{e}-06 \\
4.501472 \mathrm{e}-06\end{array}$ & $\begin{array}{l}1.987713 \mathrm{e}-04 \\
1.979938 \mathrm{e}-04 \\
2.025831 \mathrm{e}-04\end{array}$ & $\begin{array}{l}3.264800 \mathrm{e}-05 \\
3.265449 \mathrm{e}-05 \\
3.223369 \mathrm{e}-05\end{array}$ & $\begin{array}{l}1.290198 \mathrm{e}-03 \\
1.300979 \mathrm{e}-03 \\
1.332909 \mathrm{e}-03\end{array}$ & $\begin{array}{l}3.242831 \mathrm{e}-05 \\
3.238798 \mathrm{e}-05 \\
3.187756 \mathrm{e}-05\end{array}$ & $\begin{array}{l}1.301986 \mathrm{e}-03 \\
1.293037 \mathrm{e}-03 \\
1.279723 \mathrm{e}-03\end{array}$ \\
\hline \multirow{3}{*}{50} & 0.5 & $\begin{array}{c}0.8 \\
1.2 \\
3\end{array}$ & $\begin{array}{l}2.190594 \mathrm{e}-05 \\
2.188865 \mathrm{e}-05 \\
2.180402 \mathrm{e}-05\end{array}$ & $\begin{array}{l}3.945011 \mathrm{e}-06 \\
3.940208 \mathrm{e}-06 \\
3.922342 \mathrm{e}-06\end{array}$ & $\begin{array}{l}5.619432 \mathrm{e}-05 \\
5.664369 \mathrm{e}-05 \\
5.677703 \mathrm{e}-05\end{array}$ & $\begin{array}{l}3.941056 \mathrm{e}-06 \\
3.939030 \mathrm{e}-06 \\
3.926707 \mathrm{e}-06\end{array}$ & $\begin{array}{l}5.571378 \mathrm{e}-05 \\
5.599055 \mathrm{e}-05 \\
5.580299 \mathrm{e}-05\end{array}$ & $\begin{array}{l}2.806457 \mathrm{e}-05 \\
2.801748 \mathrm{e}-05 \\
2.785678 \mathrm{e}-05\end{array}$ & $\begin{array}{l}3.911557 \mathrm{e}-04 \\
3.945221 \mathrm{e}-04 \\
3.968745 \mathrm{e}-04\end{array}$ & $\begin{array}{l}2.798915 \mathrm{e}-05 \\
2.799171 \mathrm{e}-05 \\
2.793525 \mathrm{e}-05\end{array}$ & $\begin{array}{l}3.842820 \mathrm{e}-04 \\
3.852879 \mathrm{e}-04 \\
3.830106 \mathrm{e}-04\end{array}$ \\
\hline & 1.5 & $\begin{array}{c}0.8 \\
1.2 \\
3\end{array}$ & $\begin{array}{l}1.021647 \mathrm{e}-05 \\
1.013936 \mathrm{e}-05 \\
1.007830 \mathrm{e}-05\end{array}$ & $\begin{array}{l}1.839912 \mathrm{e}-06 \\
1.826681 \mathrm{e}-06 \\
1.816025 \mathrm{e}-06\end{array}$ & $\begin{array}{l}1.020488 \mathrm{e}-04 \\
1.028249 \mathrm{e}-04 \\
1.036135 \mathrm{e}-04\end{array}$ & $\begin{array}{l}1.837664 \mathrm{e}-06 \\
1.824142 \mathrm{e}-06 \\
1.812857 \mathrm{e}-06\end{array}$ & $\begin{array}{l}1.020963 \mathrm{e}-04 \\
1.021440 \mathrm{e}-04 \\
1.013372 \mathrm{e}-04\end{array}$ & $\begin{array}{l}1.309096 \mathrm{e}-05 \\
1.299452 \mathrm{e}-05 \\
1.292019 \mathrm{e}-05\end{array}$ & $\begin{array}{l}6.910311 \mathrm{e}-04 \\
6.974452 \mathrm{e}-04 \\
7.082533 \mathrm{e}-04\end{array}$ & $\begin{array}{l}1.305517 \mathrm{e}-05 \\
1.295514 \mathrm{e}-05 \\
1.287233 \mathrm{e}-05\end{array}$ & $\begin{array}{l}6.910104 \mathrm{e}-04 \\
6.878782 \mathrm{e}-04 \\
6.777248 \mathrm{e}-04\end{array}$ \\
\hline & 5 & $\begin{array}{c}0.8 \\
1.2 \\
3 \\
\end{array}$ & $\begin{array}{l}1.254817 \mathrm{e}-05 \\
1.253844 \mathrm{e}-05 \\
1.240020 \mathrm{e}-05\end{array}$ & $\begin{array}{l}2.259857 \mathrm{e}-06 \\
2.258839 \mathrm{e}-06 \\
2.234872 \mathrm{e}-06\end{array}$ & $\begin{array}{l}9.834443 \mathrm{e}-05 \\
9.853752 \mathrm{e}-05 \\
9.947852 \mathrm{e}-05\end{array}$ & $\begin{array}{l}2.256976 \mathrm{e}-06 \\
2.255126 \mathrm{e}-06 \\
2.229040 \mathrm{e}-06\end{array}$ & $\begin{array}{l}9.862793 \mathrm{e}-05 \\
9.841166 \mathrm{e}-05 \\
9.841283 \mathrm{e}-05\end{array}$ & $\begin{array}{l}1.608551 \mathrm{e}-05 \\
1.607926 \mathrm{e}-05 \\
1.591435 \mathrm{e}-05\end{array}$ & $\begin{array}{l}6.675013 \mathrm{e}-04 \\
6.701761 \mathrm{e}-04 \\
6.790319 \mathrm{e}-04\end{array}$ & $\begin{array}{l}1.602323 \mathrm{e}-05 \\
1.600500 \mathrm{e}-05 \\
1.581577 \mathrm{e}-05\end{array}$ & $\begin{array}{l}6.710974 \mathrm{e}-04 \\
6.682370 \mathrm{e}-04 \\
6.645339 \mathrm{e}-04\end{array}$ \\
\hline \multirow{3}{*}{100} & 0.5 & $\begin{array}{c}0.8 \\
1.2 \\
3 \\
\end{array}$ & $\begin{array}{l}1.086470 \mathrm{e}-05 \\
1.085740 \mathrm{e}-05 \\
1.082804 \mathrm{e}-05\end{array}$ & $\begin{array}{l}1.955930 \mathrm{e}-06 \\
1.954564 \mathrm{e}-06 \\
1.948627 \mathrm{e}-06\end{array}$ & $\begin{array}{l}2.800417 \mathrm{e}-05 \\
2.807958 \mathrm{e}-05 \\
2.819771 \mathrm{e}-05\end{array}$ & $\begin{array}{l}1.954938 \mathrm{e}-06 \\
1.953656 \mathrm{e}-06 \\
1.949811 \mathrm{e}-06\end{array}$ & $\begin{array}{l}2.788697 \mathrm{e}-05 \\
2.790925 \mathrm{e}-05 \\
2.790458 \mathrm{e}-05\end{array}$ & $\begin{array}{l}1.391383 \mathrm{e}-05 \\
1.389679 \mathrm{e}-05 \\
1.384806 \mathrm{e}-05\end{array}$ & $\begin{array}{l}1.957953 \mathrm{e}-04 \\
1.965380 \mathrm{e}-04 \\
1.978411 \mathrm{e}-04\end{array}$ & $\begin{array}{l}1.389559 \mathrm{e}-05 \\
1.389022 \mathrm{e}-05 \\
1.386628 \mathrm{e}-05\end{array}$ & $\begin{array}{l}1.940039 \mathrm{e}-04 \\
1.939673 \mathrm{e}-04 \\
1.934434 \mathrm{e}-04\end{array}$ \\
\hline & 1.5 & $\begin{array}{c}0.8 \\
1.2 \\
3\end{array}$ & $\begin{array}{l}4.972686 \mathrm{e}-06 \\
4.965874 \mathrm{e}-06 \\
4.925159 \mathrm{e}-06\end{array}$ & $\begin{array}{l}8.951313 \mathrm{e}-07 \\
8.935173 \mathrm{e}-07 \\
8.862058 \mathrm{e}-07\end{array}$ & $\begin{array}{l}5.103487 \mathrm{e}-05 \\
5.117931 \mathrm{e}-05 \\
5.158670 \mathrm{e}-05\end{array}$ & $\begin{array}{l}8.945140 \mathrm{e}-07 \\
8.926581 \mathrm{e}-07 \\
8.865111 \mathrm{e}-07\end{array}$ & $\begin{array}{l}5.107912 \mathrm{e}-05 \\
5.102645 \mathrm{e}-05 \\
5.096984 \mathrm{e}-05\end{array}$ & $\begin{array}{l}6.368128 \mathrm{e}-06 \\
6.360514 \mathrm{e}-06 \\
6.309925 \mathrm{e}-06\end{array}$ & $\begin{array}{l}3.508177 \mathrm{e}-04 \\
3.525401 \mathrm{e}-04 \\
3.567755 \mathrm{e}-04\end{array}$ & $\begin{array}{l}6.358611 \mathrm{e}-06 \\
6.348829 \mathrm{e}-06 \\
6.296464 \mathrm{e}-06\end{array}$ & $\begin{array}{l}3.512041 \mathrm{e}-04 \\
3.501259 \mathrm{e}-04 \\
3.478553 \mathrm{e}-04\end{array}$ \\
\hline & 5 & $\begin{array}{c}0.8 \\
1.2 \\
3\end{array}$ & $\begin{array}{l}6.102402 \mathrm{e}-06 \\
6.099390 \mathrm{e}-06 \\
6.087484 \mathrm{e}-06\end{array}$ & $\begin{array}{l}1.099102 \mathrm{e}-06 \\
1.098540 \mathrm{e}-06 \\
1.097488 \mathrm{e}-06\end{array}$ & $\begin{array}{l}4.966588 \mathrm{e}-05 \\
4.972746 \mathrm{e}-05 \\
4.990454 \mathrm{e}-05\end{array}$ & $\begin{array}{l}1.098281 \mathrm{e}-06 \\
1.098182 \mathrm{e}-06 \\
1.095179 \mathrm{e}-06\end{array}$ & $\begin{array}{l}4.976110 \mathrm{e}-05 \\
4.970260 \mathrm{e}-05 \\
4.960341 \mathrm{e}-05\end{array}$ & $\begin{array}{l}7.817211 \mathrm{e}-06 \\
7.814861 \mathrm{e}-06 \\
7.802162 \mathrm{e}-06\end{array}$ & $\begin{array}{l}3.417496 \mathrm{e}-04 \\
3.426243 \mathrm{e}-04 \\
3.448915 \mathrm{e}-04\end{array}$ & $\begin{array}{l}7.802258 \mathrm{e}-06 \\
7.796143 \mathrm{e}-06 \\
7.777991 \mathrm{e}-06\end{array}$ & $\begin{array}{l}3.430621 \mathrm{e}-04 \\
3.421922 \mathrm{e}-04 \\
3.405352 \mathrm{e}-04\end{array}$ \\
\hline
\end{tabular}

From table-IV, the Bayes risk of survival function is minimum under the SELF, LLF, WLF and PLF when $\beta<1$ and $\alpha>1$ than $\beta<1$ and $\alpha<1$ and also minimum when $\beta>1$ and $\alpha>1$ than $\beta>1$ and $\alpha<1$ for $n=25$. The same behavior of the values of scale and shape parameters of the Bayes risk of survival function is reported when $n=50$ and $n=100$. The Bayes risk of survival function is maximum under the GELF, QLF and SLLF when $\beta<1$ and $\alpha>1$ than $\beta<1$ and $\alpha<1$ and also maximum when $\beta>1$ and $\alpha>1$ than $\beta>1$ and $\alpha<1$ for $n=25$. The same behavior of the values of scale and shape parameters of the Bayes risk of survival function is reported when $n=50$ and $n=100$. The Bayes risk of survival function for the Weibull distribution using non-informative prior (Lognormal-Inverted Gamma prior) under LLF is better than other loss functions (SELF, GELF, PLF, QLF, WLF and SLLF) proposed in this study.

\begin{tabular}{|c|c|c|c|c|c|c|}
\hline & & & & & & \\
\hline$n$ & $\boldsymbol{\beta}$ & $\alpha$ & $R\left(S\left(t_{i}\right)\right)_{B Q}$ & $\boldsymbol{R}\left(\hat{\boldsymbol{S}}\left(\boldsymbol{t}_{i}\right)\right)_{B W}$ & $R\left(S\left(t_{i}\right)\right)_{B S L}$ & $\boldsymbol{R}\left(\widehat{\boldsymbol{S}}\left(\boldsymbol{t}_{i}\right)\right)_{B P}$ \\
\hline \multirow{9}{*}{25} & \multirow{3}{*}{0.5} & 0.8 & $5.611738 \mathrm{e}-04$ & $1.011509 \mathrm{e}-04$ & $6.388028 \mathrm{e}-04$ & $9.813104 \mathrm{e}-05$ \\
\hline & & 1.2 & $5.658009 \mathrm{e}-04$ & $1.007844 \mathrm{e}-04$ & $6.442237 \mathrm{e}-04$ & $9.819706 \mathrm{e}-05$ \\
\hline & & 3 & $5.710787 \mathrm{e}-04$ & $9.982486 \mathrm{e}-05$ & $6.417695 \mathrm{e}-04$ & $9.817495 \mathrm{e}-05$ \\
\hline & \multirow{3}{*}{1.5} & 0.8 & $8.976792 \mathrm{e}-04$ & $8.696211 \mathrm{e}-05$ & $1.174960 \mathrm{e}-03$ & $8.339607 e-05$ \\
\hline & & 1.2 & $9.095413 \mathrm{e}-04$ & $8.665000 \mathrm{e}-05$ & $1.184880 \mathrm{e}-03$ & $8.224876 \mathrm{e}-05$ \\
\hline & & 3 & $9.400054 \mathrm{e}-04$ & $8.673119 \mathrm{e}-05$ & $1.184434 \mathrm{e}-03$ & $8.047301 \mathrm{e}-05$ \\
\hline & \multirow{3}{*}{5} & 0.8 & $8.670335 \mathrm{e}-04$ & $1.042523 \mathrm{e}-04$ & $1.128910 \mathrm{e}-03$ & $1.004590 \mathrm{e}-04$ \\
\hline & & 1.2 & $8.751758 \mathrm{e}-04$ & $1.046617 \mathrm{e}-04$ & $1.114484 \mathrm{e}-03$ & $1.000389 \mathrm{e}-04$ \\
\hline & & 3 & $8.947433 \mathrm{e}-04$ & $1.042348 \mathrm{e}-04$ & $1.142675 \mathrm{e}-03$ & $9.787965 \mathrm{e}-05$ \\
\hline \multirow{9}{*}{50} & \multirow{3}{*}{0.5} & 0.8 & $2.888067 \mathrm{e}-04$ & $5.046323 \mathrm{e}-05$ & $3.124875 \mathrm{e}-04$ & $4.972868 \mathrm{e}-05$ \\
\hline & & 1.2 & $2.910105 \mathrm{e}-04$ & $5.033160 \mathrm{e}-05$ & $3.145780 \mathrm{e}-04$ & $4.969859 \mathrm{e}-05$ \\
\hline & & 3 & $2.933315 \mathrm{e}-04$ & $5.003618 \mathrm{e}-05$ & $3.143590 \mathrm{e}-04$ & $4.962553 \mathrm{e}-05$ \\
\hline & \multirow{3}{*}{1.5} & 0.8 & $4.879646 \mathrm{e}-04$ & $4.415692 \mathrm{e}-05$ & $5.735493 \mathrm{e}-04$ & $4.312268 \mathrm{e}-05$ \\
\hline & & 1.2 & $4.925029 \mathrm{e}-04$ & $4.402615 \mathrm{e}-05$ & $5.762170 \mathrm{e}-04$ & $4.272299 \mathrm{e}-05$ \\
\hline & & 3 & $5.024980 \mathrm{e}-04$ & $4.408501 \mathrm{e}-05$ & $5.760153 \mathrm{e}-04$ & $4.219234 \mathrm{e}-05$ \\
\hline & \multirow{3}{*}{5} & 0.8 & $4.739767 \mathrm{e}-04$ & $5.269982 \mathrm{e}-05$ & $5.528196 \mathrm{e}-04$ & $5.161720 \mathrm{e}-05$ \\
\hline & & 1.2 & $4.765257 \mathrm{e}-04$ & $5.280273 \mathrm{e}-05$ & $5.527561 \mathrm{e}-04$ & $5.147835 \mathrm{e}-05$ \\
\hline & & 3 & $4.836878 \mathrm{e}-04$ & $5.256112 \mathrm{e}-05$ & $5.556023 \mathrm{e}-04$ & $5.072663 \mathrm{e}-05$ \\
\hline \multirow{9}{*}{100} & \multirow{3}{*}{0.5} & 0.8 & $1.465937 \mathrm{e}-04$ & $2.473902 \mathrm{e}-05$ & $1.558669 \mathrm{e}-04$ & $2.454776 \mathrm{e}-05$ \\
\hline & & 1.2 & $1.472660 \mathrm{e}-04$ & $2.470494 \mathrm{e}-05$ & $1.561278 \mathrm{e}-04$ & $2.453537 \mathrm{e}-05$ \\
\hline & & 3 & $1.484581 \mathrm{e}-04$ & $2.461561 \mathrm{e}-05$ & $1.564479 \mathrm{e}-04$ & $2.448829 \mathrm{e}-05$ \\
\hline & \multirow{3}{*}{1.5} & 0.8 & $2.552897 \mathrm{e}-04$ & $2.132421 \mathrm{e}-05$ & $2.858466 \mathrm{e}-04$ & $2.104122 \mathrm{e}-05$ \\
\hline & & 1.2 & $2.569689 \mathrm{e}-04$ & $2.133196 \mathrm{e}-05$ & $2.861026 \mathrm{e}-04$ & $2.097705 \mathrm{e}-05$ \\
\hline & & 3 & $2.606638 \mathrm{e}-04$ & $2.126742 \mathrm{e}-05$ & $2.871319 \mathrm{e}-04$ & $2.074814 \mathrm{e}-05$ \\
\hline & \multirow{3}{*}{5} & 0.8 & $2.491189 \mathrm{e}-04$ & $2.551923 \mathrm{e}-05$ & $2.781605 \mathrm{e}-04$ & $2.521949 \mathrm{e}-05$ \\
\hline & & 1.2 & $2.500095 \mathrm{e}-04$ & $2.554171 \mathrm{e}-05$ & $2.781725 \mathrm{e}-04$ & $2.517685 \mathrm{e}-05$ \\
\hline & & 3 & $2.522876 \mathrm{e}-04$ & $2.557297 \mathrm{e}-05$ & $2.783801 \mathrm{e}-04$ & $2.506225 \mathrm{e}-05$ \\
\hline
\end{tabular}


The Bayes risk of survival function for the two parameter Weibull distribution using informative prior (Lognormal-Inverted Gamma prior) under various loss functions is obtained and presented in Table-V.

Table-V: Estimated Value of Bayes Risk of Survival Function Under Lognormal-Inverted Gamma Prior With Hyperparameters a =1.4, b =1.6 and c =1.9.

\begin{tabular}{|c|c|c|c|c|c|c|c|c|c|c|c|}
\hline \multirow[t]{2}{*}{$n$} & \multirow[t]{2}{*}{$\beta$} & \multirow[t]{2}{*}{$\alpha$} & \multirow{2}{*}{$R\left(\widehat{S}\left(t_{i}\right)\right)_{B S}$} & $R\left(\widehat{S}\left(t_{i}\right)\right)_{B L}$ & $R\left(\widehat{S}\left(t_{i}\right)\right)_{B G}$ & $R\left(\widehat{S}\left(t_{i}\right)\right)_{B L}$ & $R\left(\widehat{S}\left(t_{i}\right)\right)_{B G}$ & $R\left(\widehat{S}\left(t_{i}\right)\right)_{B L}$ & $R\left(\widehat{S}\left(t_{i}\right)\right)_{B G}$ & $R\left(\widehat{S}\left(t_{i}\right)\right)_{B L}$ & $\boldsymbol{R}\left(\widehat{\boldsymbol{S}}\left(\boldsymbol{t}_{\boldsymbol{i}}\right)\right)_{B G}$ \\
\hline & & & & \multicolumn{2}{|c|}{$m=k=0.6$} & \multicolumn{2}{|c|}{$m=k=-0.6$} & \multicolumn{2}{|c|}{$m=k=1.6$} & \multicolumn{2}{|c|}{$m=k=-1.6$} \\
\hline \multirow{9}{*}{25} & \multirow{3}{*}{0.5} & 0.8 & $4.294867 \mathrm{e}-05$ & $7.735872 \mathrm{e}-06$ & $1.132772 \mathrm{e}-04$ & $7.688254 \mathrm{e}-06$ & $1.060245 \mathrm{e}-04$ & $5.526294 \mathrm{e}-05$ & $8.032003 \mathrm{e}-04$ & $5.436846 \mathrm{e}-05$ & $7.100278 \mathrm{e}-04$ \\
\hline & & 1.2 & $4.284928 \mathrm{e}-05$ & $7.734880 \mathrm{e}-06$ & $1.142933 \mathrm{e}-04$ & $7.689679 \mathrm{e}-06$ & $1.063396 \mathrm{e}-04$ & $5.524605 \mathrm{e}-05$ & $8.108921 \mathrm{e}-04$ & $5.438548 \mathrm{e}-05$ & $7.107788 \mathrm{e}-04$ \\
\hline & & 3 & $4.290116 \mathrm{e}-05$ & $7.742260 \mathrm{e}-06$ & $1.145093 \mathrm{e}-04$ & $7.700603 \mathrm{e}-06$ & $1.063368 \mathrm{e}-04$ & $5.526929 \mathrm{e}-05$ & $8.138351 \mathrm{e}-04$ & $5.448960 \mathrm{e}-05$ & $7.104321 \mathrm{e}-04$ \\
\hline & \multirow{3}{*}{1.5} & 0.8 & 2.062 & 3.7140 & $0 e-04$ & $4 \mathrm{e}-06$ & $2 \mathrm{e}-04$ & $2.641711 \mathrm{e}-05$ & $1.340778 \mathrm{e}-03$ & $2.634559 \mathrm{e}-05$ & $1.344096 \mathrm{e}-03$ \\
\hline & & 1.2 & $2.046124 \mathrm{e}-05$ & $3.684826 \mathrm{e}-06$ & $2.075347 \mathrm{e}-04$ & $3.680737 \mathrm{e}-06$ & $2.074598 \mathrm{e}-04$ & $2.620736 \mathrm{e}-05$ & $1.349267 \mathrm{e}-03$ & $2.613938 \mathrm{e}-05$ & $1.345824 \mathrm{e}-03$ \\
\hline & & 3 & $2.036163 \mathrm{e}-05$ & $3.666566 \mathrm{e}-06$ & $2.096105 \mathrm{e}-04$ & $3.662554 \mathrm{e}-06$ & $2.080051 \mathrm{e}-04$ & $2.608217 \mathrm{e}-05$ & $1.365168 \mathrm{e}-03$ & $2.601009 \mathrm{e}-05$ & $1.345850 \mathrm{e}-03$ \\
\hline & \multirow{3}{*}{5} & 0.8 & $2.549949 \mathrm{e}-05$ & $4.589824 \mathrm{e}-06$ & $1.953013 \mathrm{e}-04$ & $4.589233 \mathrm{e}-06$ & $2.003055 \mathrm{e}-04$ & $3.261373 \mathrm{e}-05$ & $1.269284 \mathrm{e}-03$ & $3.260648 \mathrm{e}-05$ & $1.320266 \mathrm{e}-03$ \\
\hline & & 1.2 & $2.553117 \mathrm{e}-05$ & $4.595450 \mathrm{e}-06$ & $1.957048 \mathrm{e}-04$ & $4.595065 \mathrm{e}-06$ & $2.003004 \mathrm{e}-04$ & $3.265991 \mathrm{e}-05$ & $1.272546 \mathrm{e}-03$ & $3.264210 \mathrm{e}-05$ & $1.319207 \mathrm{e}-03$ \\
\hline & & 3 & $2.528938 \mathrm{e}-05$ & $4.552643 \mathrm{e}-06$ & $1.984237 \mathrm{e}-04$ & $4.550317 \mathrm{e}-06$ & $2.023849 \mathrm{e}-04$ & $3.235903 e-05$ & $1.287185 \mathrm{e}-03$ & $3.232522 \mathrm{e}-05$ & $1.326157 \mathrm{e}-03$ \\
\hline \multirow{9}{*}{50} & \multirow{3}{*}{0.5} & 0.8 & $2.175141 \mathrm{e}-05$ & $3.919602 \mathrm{e}-06$ & $5.606629 \mathrm{e}-05$ & $3.911033 \mathrm{e}-06$ & $5.467127 \mathrm{e}-05$ & $2.791363 \mathrm{e}-05$ & $5 e-04$ & $2.774727 \mathrm{e}-05$ & $3.743673 \mathrm{e}-04$ \\
\hline & & 1.2 & $2.173794 \mathrm{e}-05$ & $3.916655 \mathrm{e}-06$ & $5.647436 \mathrm{e}-05$ & $3.908508 \mathrm{e}-06$ & $5.497586 \mathrm{e}-05$ & $2.789112 \mathrm{e}-05$ & $3.979072 \mathrm{e}-04$ & $2.773517 \mathrm{e}-05$ & $3.759125 \mathrm{e}-04$ \\
\hline & & 3 & $2.173213 \mathrm{e}-05$ & $3.914964 \mathrm{e}-06$ & $5.660834 \mathrm{e}-05$ & $3.907973 \mathrm{e}-06$ & $5.503956 \mathrm{e}-05$ & $2.787186 \mathrm{e}-05$ & $3.991979 \mathrm{e}-04$ & $2.773882 \mathrm{e}-05$ & $3.761264 \mathrm{e}-04$ \\
\hline & \multirow{3}{*}{1.5} & 0.8 & $1.022158 \mathrm{e}-05$ & e-06 & 1.0193 & 1.8392 & 1.0219 & 1.308 & 6.8972 & $e-05$ & $6.922397 \mathrm{e}-04$ \\
\hline & & 1.2 & $1.015702 \mathrm{e}-05$ & $1.829244 \mathrm{e}-06$ & $1.024518 \mathrm{e}-04$ & $1.827561 \mathrm{e}-06$ & $1.025710 \mathrm{e}-04$ & $1.300677 \mathrm{e}-05$ & $6.925171 \mathrm{e}-04$ & $1.298724 \mathrm{e}-05$ & $6.931041 \mathrm{e}-04$ \\
\hline & & 3 & $1.013366 \mathrm{e}-05$ & $1.825119 \mathrm{e}-06$ & $1.029028 \mathrm{e}-04$ & $1.823468 \mathrm{e}-06$ & $1.026661 \mathrm{e}-04$ & $1.297746 \mathrm{e}-05$ & $6.964856 \mathrm{e}-04$ & $1.295651 \mathrm{e}-05$ & $6.924043 \mathrm{e}-04$ \\
\hline & \multirow{3}{*}{5} & 0.8 & $1.256598 \mathrm{e}-05$ & $2.261557 \mathrm{e}-06$ & $9.780056 \mathrm{e}-05$ & $2.261652 \mathrm{e}-06$ & $9.896071 \mathrm{e}-05$ & $1.608122 \mathrm{e}-05$ & 6.612 & $1.607254 \mathrm{e}-05$ & $6.763906 \mathrm{e}-04$ \\
\hline & & 1.2 & $1.256812 \mathrm{e}-05$ & $2.262636 \mathrm{e}-06$ & $9.784596 \mathrm{e}-05$ & $2.261974 \mathrm{e}-06$ & $9.891410 \mathrm{e}-05$ & $1.608558 \mathrm{e}-05$ & $6.618527 \mathrm{e}-04$ & $1.607430 \mathrm{e}-05$ & $6.757369 \mathrm{e}-04$ \\
\hline & & 3 & $1.246286 \mathrm{e}-05$ & $2.243887 \mathrm{e}-06$ & $9.850292 \mathrm{e}-05$ & $2.242619 \mathrm{e}-06$ & $9.941230 \mathrm{e}-05$ & $1.595331 \mathrm{e}-05$ & $6.661124 \mathrm{e}-04$ & $1.593660 \mathrm{e}-05$ & $6.776161 \mathrm{e}-04$ \\
\hline \multirow{9}{*}{100} & \multirow{3}{*}{0.5} & 0.8 & $1.083093 \mathrm{e}-05$ & $1.950430 \mathrm{e}-06$ & $2.800387 \mathrm{e}-05$ & $1.948333 \mathrm{e}-06$ & $2.761951 \mathrm{e}-05$ & $1.388080 \mathrm{e}-05$ & $1.970978 \mathrm{e}-04$ & $1.384231 \mathrm{e}-05$ & $1.911800 \mathrm{e}-04$ \\
\hline & & 1.2 & $1.082530 \mathrm{e}-05$ & $1.949242 \mathrm{e}-06$ & $2.806236 \mathrm{e}-05$ & $1.947419 \mathrm{e}-06$ & $2.766568 \mathrm{e}-05$ & $1.386976 \mathrm{e}-05$ & $1.975604 \mathrm{e}-04$ & $1.383481 \mathrm{e}-05$ & $1.914614 \mathrm{e}-04$ \\
\hline & & 3 & $1.081483 \mathrm{e}-05$ & $1.947642 \mathrm{e}-06$ & $2.816821 \mathrm{e}-05$ & $1.946281 \mathrm{e}-06$ & $2.773736 \mathrm{e}-05$ & $1.385571 \mathrm{e}-05$ & $1.983826 \mathrm{e}-04$ & $1.382588 \mathrm{e}-05$ & $1.918012 \mathrm{e}-04$ \\
\hline & \multirow{3}{*}{1.5} & 0.8 & $4.973736 \mathrm{e}-06$ & $8.954240 \mathrm{e}-07$ & $5.100676 \mathrm{e}-05$ & $8.948464 \mathrm{e}-07$ & $5.110315 \mathrm{e}-05$ & $6.367743 \mathrm{e}-06$ & $3.504399 \mathrm{e}-04$ & $6.362196 \mathrm{e}-06$ & $3.515595 \mathrm{e}-04$ \\
\hline & & 1.2 & $4.970180 \mathrm{e}-06$ & $8.941793 \mathrm{e}-07$ & $5.108258 \mathrm{e}-05$ & $8.935392 \mathrm{e}-07$ & $5.113508 \mathrm{e}-05$ & $6.363279 \mathrm{e}-06$ & $3.511241 \mathrm{e}-04$ & $6.356882 \mathrm{e}-06$ & $3.516177 \mathrm{e}-04$ \\
\hline & & 3 & $4.938645 \mathrm{e}-06$ & $8.882994 \mathrm{e}-07$ & $5.137892 \mathrm{e}-05$ & $8.890277 \mathrm{e}-07$ & $5.133142 \mathrm{e}-05$ & $6.323308 \mathrm{e}-06$ & $3.531873 \mathrm{e}-04$ & $6.317309 \mathrm{e}-06$ & $3.522757 \mathrm{e}-04$ \\
\hline & \multirow{3}{*}{5} & 0.8 & $6.106719 \mathrm{e}-06$ & $1.099295 \mathrm{e}-06$ & $4.950407 \mathrm{e}-05$ & $1.099338 \mathrm{e}-06$ & $4.986329 \mathrm{e}-05$ & $7.815825 \mathrm{e}-06$ & $3.397277 \mathrm{e}-04$ & $7.814544 \mathrm{e}-06$ & $3.447729 \mathrm{e}-04$ \\
\hline & & 1.2 & $6.106668 \mathrm{e}-06$ & $1.099300 \mathrm{e}-06$ & $4.952132 \mathrm{e}-05$ & $1.099906 \mathrm{e}-06$ & $4.985326 \mathrm{e}-05$ & $7.816686 \mathrm{e}-06$ & $3.399450 \mathrm{e}-04$ & $7.813417 \mathrm{e}-06$ & $3.445950 \mathrm{e}-04$ \\
\hline & & 3 & $6.103357 \mathrm{e}-06$ & $1.099527 \mathrm{e}-06$ & $4.962063 \mathrm{e}-05$ & $1.098603 \mathrm{e}-06$ & $4.989007 \mathrm{e}-05$ & $7.811910 \mathrm{e}-06$ & $3.408419 \mathrm{e}-04$ & $7.808576 \mathrm{e}-06$ & $3.446167 \mathrm{e}-04$ \\
\hline
\end{tabular}

From table-V,the Bayes risk of survival function is minimum under the SELF, LLF, WLF and PLF when $\beta<1$ and $\alpha>1$ than $\beta<1$ and $\alpha<1$ and also minimum when $\beta>1$ and $\alpha>1$ than $\beta>1$ and $\alpha<1$ for $\mathrm{n}=25$. The same behavior of the values of scale and shape parameters of the Bayes risk of survival function is reported when $n=50$ and $n=100$. The Bayes risk of survival function is maximum under the GELF, QLF and SLLF when $\beta<1$ and $\alpha>1$ than $\beta<1$ and $\alpha<1$ and also maximum when $\beta>1$ and $\alpha>1$ than $\beta>1$ and $\alpha<1$ for $\mathrm{n}=25$. The same behavior of the values of scale and shape parameters of the Bayes risk of survival function is reported when $n=50$ and $n=100$. The Bayes risk of survival function for the Weibull distribution using non-informative prior (Lognormal-Inverted Gamma prior) under LLF is better than other loss functions (SELF, GELF, PLF, QLF, WLF and SLLF) proposed in this study.

Table-V (Continued)

\begin{tabular}{|c|c|c|c|c|c|c|}
\hline$n$ & $\boldsymbol{\beta}$ & $\alpha$ & $R\left(\widehat{S}\left(t_{i}\right)\right)_{B O}$ & $\boldsymbol{R}\left(\widehat{\boldsymbol{S}}\left(\boldsymbol{t}_{i}\right)\right)_{B W}$ & $R\left(\widehat{S}\left(t_{i}\right)\right)_{B S L}$ & $\boldsymbol{R}\left(\widehat{\boldsymbol{S}}\left(\boldsymbol{t}_{i}\right)\right)_{B P}$ \\
\hline \multirow{3}{*}{25} & 0.5 & $\begin{array}{l}0.8 \\
1.2 \\
3\end{array}$ & $\begin{array}{l}5.822385 \mathrm{e}-04 \\
5.858339 \mathrm{e}-04 \\
5.882179 \mathrm{e}-04\end{array}$ & $\begin{array}{l}9.995195 \mathrm{e}-05 \\
9.974393 \mathrm{e}-05 \\
9.971415 \mathrm{e}-05\end{array}$ & $\begin{array}{l}6.115756 \mathrm{e}-04 \\
6.150719 \mathrm{e}-04 \\
6.154634 \mathrm{e}-04\end{array}$ & $\begin{array}{l}9.419329 \mathrm{e}-05 \\
9.407921 \mathrm{e}-05 \\
9.421474 \mathrm{e}-05\end{array}$ \\
\hline & 1.5 & $\begin{array}{l}0.8 \\
1.2 \\
3\end{array}$ & $\begin{array}{l}8.951005 \mathrm{e}-04 \\
8.973514 \mathrm{e}-04 \\
9.077615 \mathrm{e}-04\end{array}$ & $\begin{array}{l}8.678783 \mathrm{e}-05 \\
8.628051 \mathrm{e}-05 \\
8.612902 \mathrm{e}-05\end{array}$ & $\begin{array}{l}1.174913 \mathrm{e}-03 \\
1.185865 \mathrm{e}-03 \\
1.193381 \mathrm{e}-03\end{array}$ & $\begin{array}{l}8.362525 \mathrm{e}-05 \\
8.296456 \mathrm{e}-05 \\
8.238772 \mathrm{e}-05\end{array}$ \\
\hline & 5 & $\begin{array}{l}0.8 \\
1.2 \\
3\end{array}$ & $\begin{array}{l}8.519704 \mathrm{e}-04 \\
8.542085 \mathrm{e}-04 \\
8.601286 \mathrm{e}-04\end{array}$ & $\begin{array}{l}1.032314 \mathrm{e}-04 \\
1.034347 \mathrm{e}-04 \\
1.026644 \mathrm{e}-04\end{array}$ & $\begin{array}{l}1.126134 \mathrm{e}-03 \\
1.127371 \mathrm{e}-03 \\
1.143324 \mathrm{e}-03\end{array}$ & $\begin{array}{l}1.015897 \mathrm{e}-04 \\
1.016047 \mathrm{e}-04 \\
1.004840 \mathrm{e}-04\end{array}$ \\
\hline 50 & 0.5 & $\begin{array}{l}0.8 \\
1.2 \\
3\end{array}$ & $\begin{array}{l}2.946904 \mathrm{e}-04 \\
2.963490 \mathrm{e}-04 \\
2.974231 \mathrm{e}-04\end{array}$ & $\begin{array}{l}5.036961 \mathrm{e}-05 \\
5.029301 \mathrm{e}-05 \\
5.024259 \mathrm{e}-05\end{array}$ & $\begin{array}{l}3.089724 \mathrm{e}-04 \\
3.110136 \mathrm{e}-04 \\
3.763481 \mathrm{e}-04\end{array}$ & $\begin{array}{l}4.894118 \mathrm{e}-05 \\
4.888234 \mathrm{e}-05 \\
4.888059 \mathrm{e}-05\end{array}$ \\
\hline
\end{tabular}




\begin{tabular}{|c|c|c|c|c|c|c|}
\hline & 1.5 & $\begin{array}{l}0.8 \\
1.2 \\
3\end{array}$ & $\begin{array}{l}4.868932 \mathrm{e}-04 \\
4.880620 \mathrm{e}-04 \\
4.911061 \mathrm{e}-04\end{array}$ & $\begin{array}{l}4.408857 \mathrm{e}-05 \\
4.389761 \mathrm{e}-05 \\
4.388620 \mathrm{e}-05\end{array}$ & $\begin{array}{l}5.734934 \mathrm{e}-04 \\
5.763831 \mathrm{e}-04 \\
5.779676 \mathrm{e}-04\end{array}$ & $\begin{array}{l}4.321696 \mathrm{e}-05 \\
4.297189 \mathrm{e}-05 \\
4.282685 \mathrm{e}-05\end{array}$ \\
\hline & 5 & $\begin{array}{l}0.8 \\
1.2 \\
3\end{array}$ & $\begin{array}{l}4.685229 \mathrm{e}-04 \\
4.690721 \mathrm{e}-04 \\
4.717669 \mathrm{e}-04\end{array}$ & $\begin{array}{l}5.239513 \mathrm{e}-05 \\
5.243924 \mathrm{e}-05 \\
5.210451 \mathrm{e}-05\end{array}$ & $\begin{array}{l}5.521475 \mathrm{e}-04 \\
5.521594 \mathrm{e}-04 \\
5.556563 \mathrm{e}-04\end{array}$ & $\begin{array}{l}5.197276 \mathrm{e}-05 \\
5.196115 \mathrm{e}-05 \\
5.151847 \mathrm{e}-05\end{array}$ \\
\hline \multirow{2}{*}{100} & 0.5 & $\begin{array}{l}0.8 \\
1.2 \\
3\end{array}$ & $\begin{array}{l}1.484520 \mathrm{e}-04 \\
1.488216 \mathrm{e}-04 \\
1.494309 \mathrm{e}-04\end{array}$ & $\begin{array}{l}2.472081 \mathrm{e}-05 \\
2.469916 \mathrm{e}-05 \\
2.466587 \mathrm{e}-05\end{array}$ & $\begin{array}{l}1.550801 \mathrm{e}-04 \\
1.553624 \mathrm{e}-04 \\
1.558719 \mathrm{e}-04\end{array}$ & $\begin{array}{l}2.435638 \mathrm{e}-05 \\
2.434111 \mathrm{e}-05 \\
2.431485 \mathrm{e}-05\end{array}$ \\
\hline & 5 & $\begin{array}{l}0.8 \\
1.2 \\
3 \\
\end{array}$ & $\begin{array}{l}2.471615 \mathrm{e}-04 \\
2.473705 \mathrm{e}-04 \\
2.481564 \mathrm{e}-04\end{array}$ & $\begin{array}{l}2.543697 \mathrm{e}-05 \\
2.544381 \mathrm{e}-05 \\
2.544943 \mathrm{e}-05\end{array}$ & $\begin{array}{l}2.779824 \mathrm{e}-04 \\
2.780049 \mathrm{e}-04 \\
2.783849 \mathrm{e}-04\end{array}$ & $\begin{array}{l}2.531849 \mathrm{e}-05 \\
2.531043 \mathrm{e}-05 \\
2.528283 \mathrm{e}-05\end{array}$ \\
\hline
\end{tabular}

The Bayes risk of survival function for the two parameter Weibull distribution using informative prior (Gamma-Gamma prior) under various loss functions is obtained and presented in Table-VI.

Table-VI: Estimated Value of Bayes Risk of Survival Function Under Gamma-Gamma Prior With Hyperparameters $\mathbf{a}_{1}=2, b_{1}=0.2, c_{1}=0.8$ and $d_{1}=4$.

\begin{tabular}{|c|c|c|c|c|c|c|c|c|c|c|c|}
\hline \multirow{2}{*}{$n$} & \multirow{2}{*}{$\boldsymbol{\beta}$} & \multirow{2}{*}{$\alpha$} & \multirow{2}{*}{$R\left(\widehat{S}\left(t_{i}\right)\right)_{B S}$} & $\boldsymbol{R}\left(\widehat{\boldsymbol{S}}\left(\boldsymbol{t}_{i}\right)\right)_{B L}$ & $\boldsymbol{R}\left(\widehat{\boldsymbol{S}}\left(\boldsymbol{t}_{\boldsymbol{i}}\right)\right)_{B G}$ & $\boldsymbol{R}\left(\widehat{\boldsymbol{S}}\left(\boldsymbol{t}_{i}\right)\right)_{B L}$ & $\boldsymbol{R}\left(\widehat{\boldsymbol{S}}\left(\boldsymbol{t}_{\boldsymbol{i}}\right)\right)_{B G}$ & $\boldsymbol{R}\left(\widehat{\boldsymbol{S}}\left(\boldsymbol{t}_{\boldsymbol{i}}\right)\right)_{B L}$ & $\boldsymbol{R}\left(\widehat{\boldsymbol{S}}\left(\boldsymbol{t}_{\boldsymbol{i}}\right)\right)_{B G}$ & $\boldsymbol{R}\left(\widehat{\boldsymbol{S}}\left(\boldsymbol{t}_{\boldsymbol{i}}\right)\right)_{B L}$ & $\boldsymbol{R}\left(\widehat{\boldsymbol{S}}\left(\boldsymbol{t}_{\boldsymbol{i}}\right)\right)_{B G}$ \\
\hline & & & & \multicolumn{2}{|c|}{$m=k=0.6$} & \multicolumn{2}{|c|}{$m=k=-0.6$} & \multicolumn{2}{|c|}{$m=k=1.6$} & \multicolumn{2}{|c|}{$m=k=-1.6$} \\
\hline \multirow{9}{*}{25} & & 0.8 & $4.014512 \mathrm{e}-05$ & $7.267551 \mathrm{e}-06$ & $1.099770 \mathrm{e}-04$ & $7.185055 \mathrm{e}-06$ & $1.019410 \mathrm{e}-04$ & $5.217474 \mathrm{e}-05$ & $7.895477 \mathrm{e}-04$ & $5.061035 \mathrm{e}-05$ & $6.809573 \mathrm{e}-04$ \\
\hline & 0.5 & 1.2 & $4.027534 \mathrm{e}-05$ & $7.290443 \mathrm{e}-06$ & $1.112081 \mathrm{e}-04$ & $7.208658 \mathrm{e}-06$ & $1.025703 \mathrm{e}-04$ & $5.233294 \mathrm{e}-05$ & $7.976922 \mathrm{e}-04$ & $5.078137 \mathrm{e}-05$ & $6.837316 \mathrm{e}-04$ \\
\hline & & 3 & $4.069351 \mathrm{e}-05$ & $7.363594 \mathrm{e}-06$ & $1.116340 \mathrm{e}-04$ & $7.285919 \mathrm{e}-06$ & $1.026574 \mathrm{e}-04$ & $5.282508 \mathrm{e}-05$ & $8.030611 \mathrm{e}-04$ & $5.134678 \mathrm{e}-05$ & $6.839006 \mathrm{e}-04$ \\
\hline & & 0.8 & 1.896 & $3.428806 \mathrm{e}-06$ & $2.071465 \mathrm{e}-04$ & $3.397341 \mathrm{e}-06$ & $1.998796 \mathrm{e}-04$ & $2.456866 \mathrm{e}-05$ & $1.365762 \mathrm{e}-03$ & $2.397245 \mathrm{e}-05$ & $1.296525 \mathrm{e}-03$ \\
\hline & 1.5 & 1.2 & $1.885366 \mathrm{e}-05$ & $3.409099 \mathrm{e}-06$ & $2.090607 \mathrm{e}-04$ & $3.378113 \mathrm{e}-06$ & $2.008262 \mathrm{e}-04$ & $2.388645 \mathrm{e}-05$ & $1.374773 \mathrm{e}-03$ & $2.384107 \mathrm{e}-05$ & $1.297732 \mathrm{e}-03$ \\
\hline & & 3 & $1.876542 \mathrm{e}-05$ & $3.393111 \mathrm{e}-06$ & $2.110690 \mathrm{e}-04$ & $3.362494 \mathrm{e}-06$ & $2.005352 \mathrm{e}-04$ & $2.431437 \mathrm{e}-05$ & $1.393712 \mathrm{e}-03$ & $2.372522 \mathrm{e}-05$ & $1.292179 \mathrm{e}-03$ \\
\hline & & 0.8 & $2.538035 \mathrm{e}-05$ & $4.574981 \mathrm{e}-06$ & $1.960506 \mathrm{e}-04$ & $4.561282 \mathrm{e}-06$ & $1.994919 \mathrm{e}-04$ & $3.258980 \mathrm{e}-05$ & $1.273065 \mathrm{e}-03$ & $3.232913 \mathrm{e}-05$ & $1.315436 \mathrm{e}-03$ \\
\hline & 5 & 1.2 & $2.540639 \mathrm{e}-05$ & $4.579818 \mathrm{e}-06$ & $1.963520 \mathrm{e}-04$ & $4.565893 \mathrm{e}-06$ & $1.995159 \mathrm{e}-04$ & $3.262703 \mathrm{e}-05$ & $1.275417 \mathrm{e}-03$ & $3.236029 \mathrm{e}-05$ & $1.315022 \mathrm{e}-03$ \\
\hline & & 3 & $2.518159 \mathrm{e}-05$ & $4.538505 \mathrm{e}-06$ & $1.988430 \mathrm{e}-04$ & $4.525768 \mathrm{e}-06$ & $2.016555 \mathrm{e}-04$ & $3.232420 \mathrm{e}-05$ & $1.287282 \mathrm{e}-03$ & $3.208758 \mathrm{e}-05$ & $1.323838 \mathrm{e}-03$ \\
\hline \multirow{9}{*}{50} & & 0.8 & $2.102237 \mathrm{e}-05$ & $3.794868 \mathrm{e}-06$ & $+2 \mathrm{e}-05$ & $3.772967 \mathrm{e}-06$ & $5.316186 \mathrm{e}-05$ & $2.710846 \mathrm{e}-05$ & $3.915078 \mathrm{e}-04$ & $2.669731 \mathrm{e}-05$ & $3.628468 \mathrm{e}-04$ \\
\hline & 0.5 & 1.2 & $2.103552 \mathrm{e}-05$ & $3.796853 \mathrm{e}-06$ & $5.546040 \mathrm{e}-05$ & $3.775593 \mathrm{e}-06$ & $5.349458 \mathrm{e}-05$ & $2.712162 \mathrm{e}-05$ & $3.944182 \mathrm{e}-04$ & $2.671834 \mathrm{e}-05$ & $3.646557 \mathrm{e}-04$ \\
\hline & & 3 & $2.112290 \mathrm{e}-05$ & $3.811691 \mathrm{e}-06$ & $5.564703 \mathrm{e}-05$ & $3.792058 \mathrm{e}-06$ & $5.358071 \mathrm{e}-05$ & $2.721808 \mathrm{e}-05$ & $3.963086 \mathrm{e}-04$ & $2.671834 \mathrm{e}-05$ & $3.649819 \mathrm{e}-04$ \\
\hline & & 0.8 & $9.870592 \mathrm{e}-06$ & $1.781341 \mathrm{e}-06$ & 1.022 & 1.772 & e-04 & 1.271 & $8 \mathrm{e}-04$ & $9 e-05$ & $6.791504 \mathrm{e}-04$ \\
\hline & 1.5 & 1.2 & $9.817194 \mathrm{e}-06$ & $1.771760 \mathrm{e}-06$ & $1.027643 \mathrm{e}-04$ & $1.762880 \mathrm{e}-06$ & $1.011384 \mathrm{e}-04$ & $1.264388 \mathrm{e}-05$ & $6.999355 \mathrm{e}-04$ & $1.248478 \mathrm{e}-05$ & $6.797878 \mathrm{e}-04$ \\
\hline & & 3 & $9.789390 \mathrm{e}-06$ & $1.766910 \mathrm{e}-06$ & $1.031923 \mathrm{e}-04$ & $1.757944 \mathrm{e}-06$ & $1.010278 \mathrm{e}-04$ & $1.260862 \mathrm{e}-05$ & $7.046683 \mathrm{e}-04$ & $1.244786 \mathrm{e}-05$ & $6.774036 \mathrm{e}-04$ \\
\hline & & 0.8 & $1.256182 \mathrm{e}-05$ & $2.261842 \mathrm{e}-06$ & 9.778 & 2.259 & $20 \mathrm{e}-05$ & $1.609493 \mathrm{e}-05$ & $6.606536 \mathrm{e}-04$ & $56 e-05$ & $6.768761 \mathrm{e}-04$ \\
\hline & 5 & 1.2 & $1.256331 \mathrm{e}-05$ & $2.262739 \mathrm{e}-06$ & $9.781208 \mathrm{e}-05$ & $2.259938 \mathrm{e}-06$ & $9.893377 \mathrm{e}-05$ & $1.609812 \mathrm{e}-05$ & $6.610568 \mathrm{e}-04$ & $1.605026 \mathrm{e}-05$ & $6.763769 \mathrm{e}-04$ \\
\hline & & 3 & $1.246152 \mathrm{e}-05$ & $2.244410 \mathrm{e}-06$ & $9.844368 \mathrm{e}-05$ & $2.241529 \mathrm{e}-06$ & $9.945515 \mathrm{e}-05$ & $1.596548 \mathrm{e}-05$ & $6.650693 \mathrm{e}-04$ & $1.592078 \mathrm{e}-05$ & $6.785324 \mathrm{e}-04$ \\
\hline \multirow{9}{*}{100} & & 0.8 & $1.065015 \mathrm{e}-05$ & $1.919665 \mathrm{e}-06$ & $2.774873 \mathrm{e}-05$ & $1.914198 \mathrm{e}-06$ & $2.721320 \mathrm{e}-05$ & $1.368377 \mathrm{e}-05$ & $1.962578 \mathrm{e}-04$ & $1.357824 \mathrm{e}-05$ & $1.878128 \mathrm{e}-04$ \\
\hline & 0.5 & 1.2 & $1.064880 \mathrm{e}-05$ & $1.919424 \mathrm{e}-06$ & $2.780955 \mathrm{e}-05$ & $1.913719 \mathrm{e}-06$ & $2.726418 \mathrm{e}-05$ & $1.367870 \mathrm{e}-05$ & $1.967178 \mathrm{e}-04$ & $1.357567 \mathrm{e}-05$ & $1.881439 \mathrm{e}-04$ \\
\hline & & 3 & $1.066076 \mathrm{e}-05$ & $1.921407 \mathrm{e}-06$ & $2.792810 \mathrm{e}-05$ & $1.916599 \mathrm{e}-06$ & $2.733840 \mathrm{e}-05$ & $1.369197 \mathrm{e}-05$ & $1.977017 \mathrm{e}-04$ & $1.359626 \mathrm{e}-05$ & $1.884710 \mathrm{e}-04$ \\
\hline & & 0.8 & $4.884211 \mathrm{e}-06$ & $8.801920 \mathrm{e}-07$ & $5.111645 \mathrm{e}-05$ & $8.777760 \mathrm{e}-07$ & $5.070324 \mathrm{e}-05$ & $6.272585 \mathrm{e}-06$ & $3.530239 \mathrm{e}-04$ & $6.228581 \mathrm{e}-06$ & $3.472352 \mathrm{e}-04$ \\
\hline & 1.5 & 1.2 & $4.880604 \mathrm{e}-06$ & $8.792664 \mathrm{e}-07$ & $5.117982 \mathrm{e}-05$ & $8.764255 \mathrm{e}-07$ & $5.073470 \mathrm{e}-05$ & $6.268356 \mathrm{e}-06$ & $3.535903 \mathrm{e}-04$ & $6.223300 \mathrm{e}-06$ & $3.473457 \mathrm{e}-04$ \\
\hline & & 3 & $4.848956 \mathrm{e}-06$ & $8.733570 \mathrm{e}-07$ & $5.147899 \mathrm{e}-05$ & $8.718754 \mathrm{e}-07$ & $5.086536 \mathrm{e}-05$ & $6.228071 \mathrm{e}-06$ & $3.559498 \mathrm{e}-04$ & $6.183799 \mathrm{e}-06$ & $3.474042 \mathrm{e}-04$ \\
\hline & & 0.8 & $6.104964 \mathrm{e}-06$ & $1.099159 \mathrm{e}-06$ & $4.951060 \mathrm{e}-05$ & $1.098681 \mathrm{e}-06$ & $4.985595 \mathrm{e}-05$ & $7.818506 \mathrm{e}-06$ & $3.396869 \mathrm{e}-04$ & $7.807270 \mathrm{e}-06$ & $3.447843 \mathrm{e}-04$ \\
\hline & 5 & 1.2 & $6.104720 \mathrm{e}-06$ & $1.099334 \mathrm{e}-06$ & $4.952242 \mathrm{e}-05$ & $1.099172 \mathrm{e}-06$ & $4.984939 \mathrm{e}-05$ & $7.818775 \mathrm{e}-06$ & $3.398446 \mathrm{e}-04$ & $7.806359 \mathrm{e}-06$ & $3.446528 \mathrm{e}-04$ \\
\hline & & 3 & $6.100523 \mathrm{e}-06$ & $1.099450 \mathrm{e}-06$ & $4.963756 \mathrm{e}-05$ & $1.097833 \mathrm{e}-06$ & $4.987484 \mathrm{e}-05$ & $7.813176 \mathrm{e}-06$ & $3.409476 \mathrm{e}-04$ & $7.799654 \mathrm{e}-06$ & $3.444989 \mathrm{e}-04$ \\
\hline
\end{tabular}

From table-VI, the Bayes risk of survival function is minimum under the SELF, LLF, WLF and PLF when $\beta<1$ and $\alpha<1$ than $\beta<1$ and $\alpha>1$ and maximum when $\beta>1$ and $\alpha<1$ than $\beta>1$ and $\alpha>1$ for $\mathrm{n}=25$. The same behavior of the values of scale and shape parameters of the Bayes risk of survival function is reported when $n=50$ and $n=100$. The Bayes risk of survival function is maximum under the GELF, QLF and SLLF when $\beta<1$ and $\alpha>1$ than $\beta<1$ and $\alpha<1$ and also maximum when $\beta>1$ and $\alpha>1$ than $\beta>1$ and $\alpha<1$ for $n=25$. The same behavior of the values of scale and shape parameters of the Bayes risk of survival function is reported when $n=50$ and $n=100$. The Bayes risk of survival function for the Weibull distribution using non-informative prior (Gamma-Gamma prior) under LLF is better than other loss functions (SELF, GELF, PLF, QLF, WLF and SLLF) proposed in this study.

\begin{tabular}{|c|c|c|c|c|c|c|}
\hline $\boldsymbol{n}$ & $\boldsymbol{\beta}$ & $\boldsymbol{\alpha}$ & $\boldsymbol{R}\left(\widehat{\boldsymbol{S}}\left(\boldsymbol{t}_{\boldsymbol{i}}\right)\right)_{\boldsymbol{B} \boldsymbol{Q}}$ & $\boldsymbol{R}\left(\widehat{\boldsymbol{S}}\left(\boldsymbol{t}_{\boldsymbol{i}}\right)\right)_{\boldsymbol{B} \boldsymbol{W}}$ & $\boldsymbol{R}\left(\widehat{\boldsymbol{S}}\left(\boldsymbol{t}_{\boldsymbol{i}}\right)\right)_{\boldsymbol{B S L}}$ & $\boldsymbol{R}\left(\widehat{\boldsymbol{S}}\left(\boldsymbol{t}_{\boldsymbol{i}}\right)\right)_{\boldsymbol{B} \boldsymbol{P}}$ \\
\hline \multirow{2}{*}{25} & \multirow{2}{*}{0.5} & 0.8 & $5.808840 \mathrm{e}-04$ & $9.561650 \mathrm{e}-05$ & $5.790195 \mathrm{e}-03$ & $8.750687 \mathrm{e}-05$ \\
& & 1.2 & $5.844166 \mathrm{e}-04$ & $9.570288 \mathrm{e}-05$ & $5.795317 \mathrm{e}-03$ & $8.767782 \mathrm{e}-05$ \\
\hline
\end{tabular}




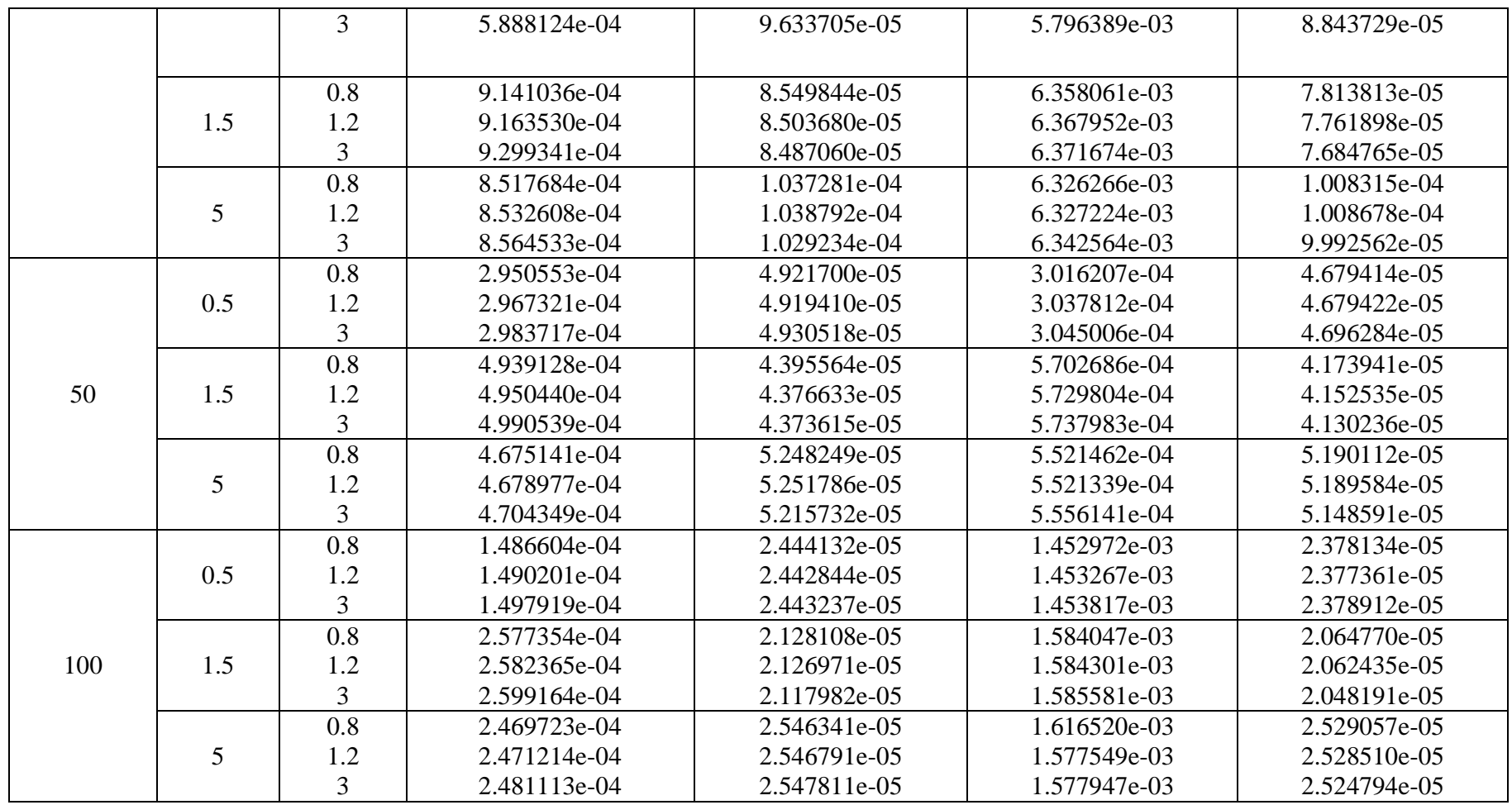

The Bayes risk of survival function for the two parameter Weibull distribution using informative prior (Gamma-Gamma prior) under various loss functions is obtained and presented in Table-VII.

Table-VII: Estimated Value of Bayes Risk of Survival Function Under Gamma-Gamma Prior With Hyperparameters $\mathbf{a}_{\mathbf{1}}=\mathbf{4}, \mathbf{b}_{\mathbf{1}}=\mathbf{0 . 5}, \mathrm{c}_{\mathbf{1}}=\mathbf{1 . 5}$ and $\boldsymbol{d}_{\mathbf{1}}=\mathbf{3}$.

\begin{tabular}{|c|c|c|c|c|c|c|c|c|c|c|c|}
\hline \multirow{2}{*}{$n$} & \multirow{2}{*}{$\boldsymbol{\beta}$} & \multirow{2}{*}{$\alpha$} & \multirow{2}{*}{$\boldsymbol{R}\left(\widehat{\boldsymbol{S}}\left(\boldsymbol{t}_{i}\right)\right)_{B S}$} & $\boldsymbol{R}\left(\widehat{\boldsymbol{S}}\left(\boldsymbol{t}_{i}\right)\right)_{B L}$ & $R\left(\widehat{S}\left(t_{i}\right)\right)_{B G}$ & $\boldsymbol{R}\left(\widehat{\boldsymbol{S}}\left(\boldsymbol{t}_{i}\right)\right)_{B L}$ & $\boldsymbol{R}\left(\widehat{\boldsymbol{S}}\left(\boldsymbol{t}_{i}\right)\right)_{B G}$ & $\boldsymbol{R}\left(\widehat{\boldsymbol{S}}\left(\boldsymbol{t}_{i}\right)\right)_{B L}$ & $\boldsymbol{R}\left(\widehat{\boldsymbol{S}}\left(\boldsymbol{t}_{i}\right)\right)_{B G}$ & $\boldsymbol{R}\left(\widehat{\boldsymbol{S}}\left(\boldsymbol{t}_{i}\right)\right)_{B L}$ & $R\left(\widehat{S}\left(t_{i}\right)\right)_{B G}$ \\
\hline & & & & \multicolumn{2}{|c|}{$m=k=0.6$} & \multicolumn{2}{|c|}{$m=k=-0.6$} & \multicolumn{2}{|c|}{$m=k=1.6$} & \multicolumn{2}{|c|}{$m=k=-1.6$} \\
\hline \multirow{3}{*}{25} & 0.5 & $\begin{array}{c}0.8 \\
1.2 \\
3\end{array}$ & $\begin{array}{l}3.984762 \mathrm{e}-05 \\
4.016202 \mathrm{e}-05 \\
4.135576 \mathrm{e}-05\end{array}$ & $\begin{array}{l}7.218465 \mathrm{e}-06 \\
7.274280 \mathrm{e}-06 \\
7.483770 \mathrm{e}-06\end{array}$ & $\begin{array}{l}1.111968 \mathrm{e}-04 \\
1.126692 \mathrm{e}-04 \\
1.141935 \mathrm{e}-04\end{array}$ & $\begin{array}{l}7.126914 \mathrm{e}-06 \\
7.184321 \mathrm{e}-06 \\
7.403464 \mathrm{e}-06\end{array}$ & $\begin{array}{l}1.077668 \mathrm{e}-04 \\
1.087596 \mathrm{e}-04 \\
1.092491 \mathrm{e}-04\end{array}$ & $\begin{array}{l}5.188595 \mathrm{e}-05 \\
5.226731 \mathrm{e}-05 \\
5.367860 \mathrm{e}-05\end{array}$ & $\begin{array}{l}7.685475 \mathrm{e}-04 \\
7.775298 \mathrm{e}-04 \\
7.914433 \mathrm{e}-04\end{array}$ & $\begin{array}{l}5.014110 \mathrm{e}-05 \\
5.055673 \mathrm{e}-05 \\
5.216857 \mathrm{e}-05\end{array}$ & $\begin{array}{l}7.263740 \mathrm{e}-04 \\
7.307375 \mathrm{e}-04 \\
7.316969 \mathrm{e}-04\end{array}$ \\
\hline & 1.5 & $\begin{array}{c}0.8 \\
1.2 \\
3 \\
\end{array}$ & $\begin{array}{l}2.034348 \mathrm{e}-05 \\
2.022698 \mathrm{e}-05 \\
2.018416 \mathrm{e}-05\end{array}$ & $\begin{array}{l}3.668997 \mathrm{e}-06 \\
3.648003 \mathrm{e}-06 \\
3.640861 \mathrm{e}-06\end{array}$ & $\begin{array}{l}1.960208 \mathrm{e}-04 \\
1.984333 \mathrm{e}-04 \\
2.041161 \mathrm{e}-04\end{array}$ & $\begin{array}{l}3.654649 \mathrm{e}-06 \\
3.633242 \mathrm{e}-06 \\
3.625009 \mathrm{e}-06\end{array}$ & $\begin{array}{l}2.099267 \mathrm{e}-04 \\
2.114879 \mathrm{e}-04 \\
2.114363 \mathrm{e}-04\end{array}$ & $\begin{array}{l}2.616645 \mathrm{e}-05 \\
2.601391 \mathrm{e}-05 \\
2.596526 \mathrm{e}-05\end{array}$ & $\begin{array}{l}1.254468 \mathrm{e}-03 \\
1.267148 \mathrm{e}-03 \\
1.310691 \mathrm{e}-03\end{array}$ & $\begin{array}{l}2.588626 \mathrm{e}-05 \\
2.574204 \mathrm{e}-05 \\
2.567999 \mathrm{e}-05\end{array}$ & $\begin{array}{l}1.407696 \mathrm{e}-03 \\
1.408835 \mathrm{e}-03 \\
1.392225 \mathrm{e}-03\end{array}$ \\
\hline & 5 & $\begin{array}{c}0.8 \\
1.2 \\
3\end{array}$ & $\begin{array}{l}2.318130 \mathrm{e}-05 \\
2.326838 \mathrm{e}-05 \\
2.292033 \mathrm{e}-05\end{array}$ & $\begin{array}{l}4.147782 \mathrm{e}-06 \\
4.163624 \mathrm{e}-06 \\
4.101280 \mathrm{e}-06\end{array}$ & $\begin{array}{l}1.653558 \mathrm{e}-04 \\
1.663707 \mathrm{e}-04 \\
1.695601 \mathrm{e}-04\end{array}$ & $\begin{array}{l}4.197408 \mathrm{e}-06 \\
4.213157 \mathrm{e}-06 \\
4.150441 \mathrm{e}-06\end{array}$ & $\begin{array}{l}1.944005 \mathrm{e}-04 \\
1.950508 \mathrm{e}-04 \\
1.986187 \mathrm{e}-04\end{array}$ & $\begin{array}{l}2.919715 \mathrm{e}-05 \\
2.931158 \mathrm{e}-05 \\
2.887436 \mathrm{e}-05\end{array}$ & $\begin{array}{l}1.055630 \mathrm{e}-03 \\
1.061603 \mathrm{e}-03 \\
1.077417 \mathrm{e}-03\end{array}$ & $\begin{array}{l}3.013978 \mathrm{e}-05 \\
3.024725 \mathrm{e}-05 \\
2.979475 \mathrm{e}-05\end{array}$ & $\begin{array}{l}1.397420 \mathrm{e}-03 \\
1.397841 \mathrm{e}-03 \\
1.408626 \mathrm{e}-03\end{array}$ \\
\hline \multirow{3}{*}{50} & 0.5 & $\begin{array}{c}0.8 \\
1.2 \\
3\end{array}$ & $\begin{array}{l}2.094366 \mathrm{e}-05 \\
2.100421 \mathrm{e}-05 \\
2.127878 \mathrm{e}-05\end{array}$ & $\begin{array}{l}3.781943 \mathrm{e}-06 \\
3.792489 \mathrm{e}-06 \\
3.839743 \mathrm{e}-06\end{array}$ & $\begin{array}{l}5.518391 \mathrm{e}-05 \\
5.567123 \mathrm{e}-05 \\
5.613668 \mathrm{e}-05\end{array}$ & $\begin{array}{l}3.757746 \mathrm{e}-06 \\
3.768925 \mathrm{e}-06 \\
3.820322 \mathrm{e}-06\end{array}$ & $\begin{array}{l}5.462398 \mathrm{e}-05 \\
5.503193 \mathrm{e}-05 \\
5.522641 \mathrm{e}-05\end{array}$ & $\begin{array}{l}2.703305 \mathrm{e}-05 \\
2.710145 \mathrm{e}-05 \\
2.741483 \mathrm{e}-05\end{array}$ & $\begin{array}{l}3.852753 \mathrm{e}-04 \\
3.884963 \mathrm{e}-04 \\
3.928289 \mathrm{e}-04\end{array}$ & $\begin{array}{l}2.657143 \mathrm{e}-05 \\
2.665721 \mathrm{e}-05 \\
2.704245 \mathrm{e}-05\end{array}$ & $\begin{array}{l}3.766294 \mathrm{e}-04 \\
3.788316 \mathrm{e}-04 \\
3.792483 \mathrm{e}-04\end{array}$ \\
\hline & 1.5 & $\begin{array}{c}0.8 \\
1.2 \\
3\end{array}$ & $\begin{array}{l}1.020149 \mathrm{e}-05 \\
1.014552 \mathrm{e}-05 \\
1.012969 \mathrm{e}-05\end{array}$ & $\begin{array}{l}1.837821 \mathrm{e}-06 \\
1.828047 \mathrm{e}-06 \\
1.825493 \mathrm{e}-06\end{array}$ & $\begin{array}{l}9.943227 \mathrm{e}-05 \\
1.001045 \mathrm{e}-04 \\
1.014657 \mathrm{e}-04\end{array}$ & $\begin{array}{l}1.834753 \mathrm{e}-06 \\
1.824656 \mathrm{e}-06 \\
1.821811 \mathrm{e}-06\end{array}$ & $\begin{array}{l}1.030500 \mathrm{e}-04 \\
1.035228 \mathrm{e}-04 \\
1.035671 \mathrm{e}-04\end{array}$ & $\begin{array}{l}1.307831 \mathrm{e}-05 \\
1.300673 \mathrm{e}-05 \\
1.298993 \mathrm{e}-05\end{array}$ & $\begin{array}{l}6.630762 \mathrm{e}-04 \\
6.671633 \mathrm{e}-04 \\
6.794194 \mathrm{e}-04\end{array}$ & $\begin{array}{l}1.302944 \mathrm{e}-05 \\
1.295834 \mathrm{e}-05 \\
1.293414 \mathrm{e}-05\end{array}$ & $\begin{array}{l}7.115028 \mathrm{e}-04 \\
7.122516 \mathrm{e}-04 \\
7.070836 \mathrm{e}-04\end{array}$ \\
\hline & 5 & $\begin{array}{c}0.8 \\
1.2 \\
3 \\
\end{array}$ & $\begin{array}{l}1.182385 \mathrm{e}-05 \\
1.184733 \mathrm{e}-05 \\
1.175207 \mathrm{e}-05\end{array}$ & $\begin{array}{l}2.120236 \mathrm{e}-06 \\
2.125342 \mathrm{e}-06 \\
2.108920 \mathrm{e}-06\end{array}$ & $\begin{array}{l}8.913055 \mathrm{e}-05 \\
8.937835 \mathrm{e}-05 \\
9.040764 \mathrm{e}-05\end{array}$ & $\begin{array}{l}2.135696 \mathrm{e}-06 \\
2.139757 \mathrm{e}-06 \\
2.122119 \mathrm{e}-06\end{array}$ & $\begin{array}{l}9.652008 \mathrm{e}-05 \\
9.665575 \mathrm{e}-05 \\
9.754150 \mathrm{e}-05\end{array}$ & $\begin{array}{l}1.499231 \mathrm{e}-05 \\
1.502406 \mathrm{e}-05 \\
1.490797 \mathrm{e}-05\end{array}$ & $\begin{array}{l}5.902475 \mathrm{e}-04 \\
5.920631 \mathrm{e}-04 \\
5.993207 \mathrm{e}-04\end{array}$ & $\begin{array}{l}1.527151 \mathrm{e}-05 \\
1.530010 \mathrm{e}-05 \\
1.517258 \mathrm{e}-05\end{array}$ & $\begin{array}{l}6.953277 \mathrm{e}-04 \\
6.952610 \mathrm{e}-04 \\
6.984471 \mathrm{e}-04\end{array}$ \\
\hline \multirow{3}{*}{100} & 0.5 & $\begin{array}{c}0.8 \\
1.2 \\
3\end{array}$ & $\begin{array}{l}1.063735 \mathrm{e}-05 \\
1.064749 \mathrm{e}-05 \\
1.070227 \mathrm{e}-05\end{array}$ & $\begin{array}{l}1.917630 \mathrm{e}-06 \\
1.919428 \mathrm{e}-06 \\
1.928920 \mathrm{e}-06\end{array}$ & $\begin{array}{l}2.772836 \mathrm{e}-05 \\
2.780902 \mathrm{e}-05 \\
2.800771 \mathrm{e}-05\end{array}$ & $\begin{array}{l}1.911633 \mathrm{e}-06 \\
1.913175 \mathrm{e}-06 \\
1.924276 \mathrm{e}-06\end{array}$ & $\begin{array}{l}2.764884 \mathrm{e}-05 \\
2.770788 \mathrm{e}-05 \\
2.780788 \mathrm{e}-05\end{array}$ & $\begin{array}{l}1.367235 \mathrm{e}-05 \\
1.368051 \mathrm{e}-05 \\
1.374261 \mathrm{e}-05\end{array}$ & $\begin{array}{l}1.938785 \mathrm{e}-04 \\
1.945153 \mathrm{e}-04 \\
1.962371 \mathrm{e}-04\end{array}$ & $\begin{array}{l}1.355639 \mathrm{e}-05 \\
1.356999 \mathrm{e}-05 \\
1.365060 \mathrm{e}-05\end{array}$ & $\begin{array}{l}1.924839 \mathrm{e}-04 \\
1.928152 \mathrm{e}-04 \\
1.930711 \mathrm{e}-04\end{array}$ \\
\hline & 1.5 & $\begin{array}{c}0.8 \\
1.2 \\
3 \\
\end{array}$ & $\begin{array}{l}4.968230 \mathrm{e}-06 \\
4.965558 \mathrm{e}-06 \\
4.936217 \mathrm{e}-06 \\
\end{array}$ & $\begin{array}{l}8.946362 \mathrm{e}-07 \\
8.936847 \mathrm{e}-07 \\
8.881665 \mathrm{e}-07 \\
\end{array}$ & $\begin{array}{l}5.030675 \mathrm{e}-05 \\
5.043082 \mathrm{e}-05 \\
5.096720 \mathrm{e}-05\end{array}$ & $\begin{array}{l}8.936279 \mathrm{e}-07 \\
8.925872 \mathrm{e}-07 \\
8.881965 \mathrm{e}-07 \\
\end{array}$ & $\begin{array}{l}5.136448 \mathrm{e}-05 \\
5.141018 \mathrm{e}-05 \\
5.159139 \mathrm{e}-05\end{array}$ & $\begin{array}{l}6.364176 \mathrm{e}-06 \\
6.361429 \mathrm{e}-06 \\
6.324989 \mathrm{e}-06 \\
\end{array}$ & $\begin{array}{l}3.424128 \mathrm{e}-04 \\
3.435033 \mathrm{e}-04 \\
3.479764 \mathrm{e}-04\end{array}$ & $\begin{array}{l}6.351311 \mathrm{e}-06 \\
6.347218 \mathrm{e}-06 \\
6.309718 \mathrm{e}-06 \\
\end{array}$ & $\begin{array}{l}3.573922 \mathrm{e}-04 \\
3.573509 \mathrm{e}-04 \\
3.567367 \mathrm{e}-04\end{array}$ \\
\hline & 5 & $\begin{array}{c}0.8 \\
1.2 \\
3\end{array}$ & $\begin{array}{l}5.927346 \mathrm{e}-06 \\
5.932501 \mathrm{e}-06 \\
5.951005 \mathrm{e}-06\end{array}$ & $\begin{array}{l}1.064800 \mathrm{e}-06 \\
1.065963 \mathrm{e}-06 \\
1.070223 \mathrm{e}-06\end{array}$ & $\begin{array}{l}4.699830 \mathrm{e}-05 \\
4.707423 \mathrm{e}-05 \\
4.746149 \mathrm{e}-05\end{array}$ & $\begin{array}{l}1.068909 \mathrm{e}-06 \\
1.070597 \mathrm{e}-06 \\
1.073164 \mathrm{e}-06\end{array}$ & $\begin{array}{l}4.936427 \mathrm{e}-05 \\
4.939839 \mathrm{e}-05 \\
4.959805 \mathrm{e}-05\end{array}$ & $\begin{array}{l}7.548170 \mathrm{e}-06 \\
7.555378 \mathrm{e}-06 \\
7.580245 \mathrm{e}-06\end{array}$ & $\begin{array}{l}3.169068 \mathrm{e}-04 \\
3.175103 \mathrm{e}-04 \\
3.205805 \mathrm{e}-04\end{array}$ & $\begin{array}{l}7.624218 \mathrm{e}-06 \\
7.629460 \mathrm{e}-06 \\
7.650778 \mathrm{e}-06\end{array}$ & $\begin{array}{l}3.516515 \mathrm{e}-04 \\
3.516204 \mathrm{e}-04 \\
3.518337 \mathrm{e}-04\end{array}$ \\
\hline
\end{tabular}

From table-VII, the Bayes risk of survival function is minimum under the SELF, LLF, WLF and PLF when $\beta<1$ and $\alpha<1$ than $\beta<1$ and $\alpha>1$ and maximum when $\beta>1$ and $\alpha<1$ than $\beta>1$ and $\alpha>1$ for $n=25$. The same behavior of the values of scale and shape 
parameters of the Bayes risk of survival function is reported when $n=50$ and $n=100$. The Bayes risk of survival function is maximum under the GELF, QLF and SLLF when $\beta<1$ and $\alpha>1$ than $\beta<1$ and $\alpha<1$ and also maximum when $\beta>1$ and $\alpha>1$ than $\beta>1$ and $\alpha<1$ for $n=25$. The same behavior of the values of scale and shape parameters of the Bayes risk of survival function is reported when $n=50$ and $n=100$. The Bayes risk of survival function for the Weibull distribution using non-informative prior (Gamma-Gamma prior) under LLF is better than other loss functions (SELF, GELF, PLF, QLF, WLF and SLLF) proposed in this study.

Table-VII (Continued)

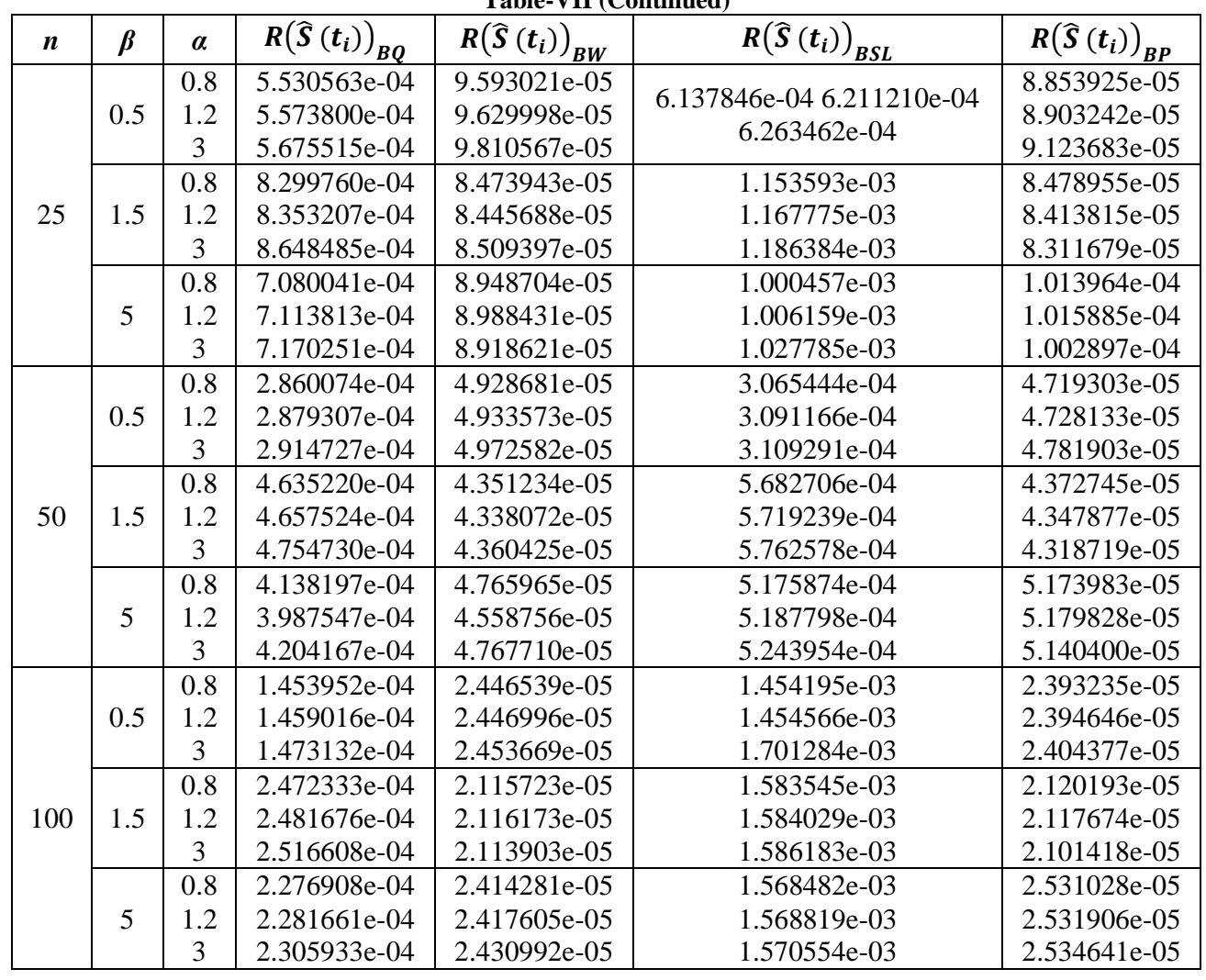

VI. CONCLUSION

In this study, we obtained the Bayes risk of survival function for the two parameter Weibull distribution using noninformative and informative priors such as Jeffrey's, Extension of Jeffrey's, Lognormal-Inverted Gamma and Gamma-Gamma with different loss functions by applying Lindley's approximation and illustrated the methodology through simulation technique. It is observed that the informative prior performed quite well than the non-informative prior. Regarding the Bayesian parametric analysis, it is observed that, from tables I to VII for the loss functions, Squared error loss, Linex loss, Precautionary loss and Weighted loss, whatever may be the $\beta$ values, the Bayes risk is less when $\alpha>1$ than $\alpha<1$ and also for the loss functions, General entropy loss, Quadratic loss and Squared logarithmic loss, the Bayes risk is minimum when $\alpha<1$ than $a>1$ and it is also true for whatever may be the hyperparameter values $(<1$ or $>1)$. In overall analysis, it is observed that whatever may be the hypaerparameters and parameter values the Bayes risk of survival function assuming Lognormal-Inverted Gamma under Linex loss function is minimum compare with assuming all other loss functions which are considered in this study. In addition to that, for all the cases in this study, it is observed that when the sample size and iteration process is increased the Bayes risk of survival function is decreased. Finally, in overall analysis, it is observed that the Bayes risk of the survival function is minimum when assuming Gamma-Gamma prior under Linex loss function compare with all other combination of priors and loss functions and it is performed well in the posterior analysis.

\section{REFERENCES}

[1] A.O.M. Ahmed and N.A. Ibrahim, "Bayesian Survival Estimator for Weibull Distribtuion with Censored Data", Journal of Applied Sciences, 11, pp: 393-396, 2011.

[2] A.O.M. Ahmed, H.S. Al-Kutubi and N.A. Ibrahim, "Comparison of the Bayesian and Maximum Likelihood Estimation for Weibull 
Distribution”, Journal of Mathematics and Statistics, 6(2), pp: 100-104, 2010.

[3] M. Aslam, S.M. Ali Kazmi, I. Ahmead and S.H. Shah, "Bayesian Estimation for Parameters of the Weibull Distribution", Sci.Int (Lahore), 26(5), pp: 1915-1920, 2014.

[4] C.B. Guure and N.A. Ibrahim, "Bayesian Analysis of the Survival Function and Failure Rate of Weibull Distribution with Censored Data", Mathematical Problems in Engineering, 2012.

[5] C.B. Guure, N.A. Ibrahim, M.B. Adam, A.O.M. Ahmed and S. Bosomprah, "Bayesian Parameter and Reliability Estimate of Weibull Failure Time Distribution," Bulletin of the Malaysian Mathematical Sciences Society, (2) 37(3), pp: 611-632, 2014.

[6] A. Lavanya and T. Leo Alexander, "Estimation of the Survival Function Under the Constant Shape Bi-Weibull Failure Distribution Based on Three Loss Functions,” International Journal of Advanced Research, 4(9), pp: 1225-1234, 2016.

[7] B.N. Pandey, N. Dwivedi and P. Bandyopadhyay, "Comparison Between Bayesian and Maximum Likelihood Estimation of the Scale Parameter in Weibull Distribution with Known Shape under Linex Loss Function”, Journal of Scientific Research, Vol 55, 2011.

[8] S.K. Sinha, "Bayes Estimation of the Reliability Function and Hazard Rate of a Weibull Failure Time Distribution", Tranbajos De Estadistica, Vol.1, No.2, pp: 47-56, 1986.

[9] G. Venkatesan and P. Saranya, "Bayesian Weibull Model in Survival Analysis", Research and Review: Journal of Statistics, 7(1), 24s-29sp, 2018a.

[10]G. Venkatesan and P. Saranya, "Bayesian Survival Analysis of Weibull Distribution Assuming Various Loss Structure", International Journal of Interdisciplinary Research and Innovations, Vol.6, issue 2, pp: 339- 353, $2018 \mathrm{~b}$.

[11]G. Venkatesan and P. Saranya, "Posterior Risk Analysis of Survival Function of Weibull Distribution", International Journal of Research and Analytical Reviews, Vol.5, issue 3, pp: 705-716, 2018c.

[12]A. Zellner, "Bayesian Estimation and Prediction using Asymmetric Loss Functions", Journal of the American Statistical Association, Vol.81, No.394, pp: 446-451, 1986.

\section{AUTHORS PROFILE}

Dr.G.Venkatesan worked as Associate professor and Head of Statistics department and Principal incharge in Government Arts College (Autonomous), Salem-636007, Tamil Nadu, India. He has 32 years of experience worked on Assistant and Associate professor in various Government colleges in Tamil Nadu, India. He has conducted various workshops, seminars and conferences and also carried out many social projects with Tamil Nadu Police department and All India Radio. He guided 56 M.Phil and Ph.D candidates. His major research fields are Bayesian Inference: Bayesian analysis of structural changes in various statistical fields, Bayesian demographic analysis. He has published 40 research papers in national and international journal.

P.Saranya did her M.Phil degree at department of Statistics, Government Arts College (Autonomous), Salem-636007 under the guidance of Dr.G.Venkatesan and also undergoing the Ph.D program. The field of her research work is Bayesian Inference and Bayesian survival analysis. She has published three research papers in an international journal. 